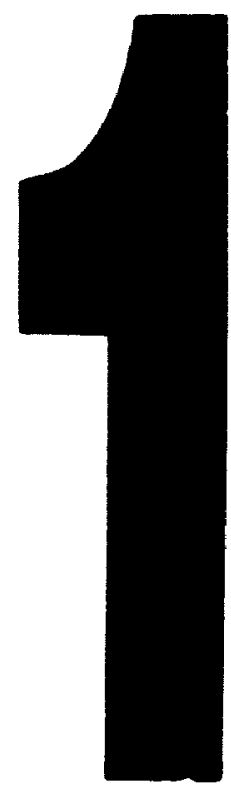

PM-1 3': $x 4$ PHOTOGRAPHIC MICROCOPY TARGET NBS $1010 a$ ANSI/ISO $\# 2$ EOUIVALENT

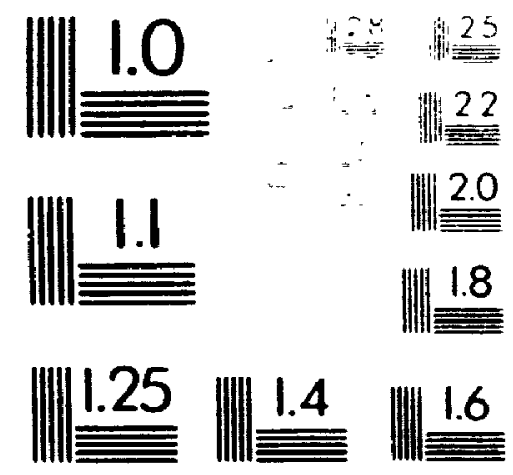

PRECISIONSM RESOLUTION TARGeTS 


\section{Transient Thermal Analysis of Printed Circuit Boards and Electronic Packages}

b:

Phanilatha Vishnubhotla

A thesis submitted to the Faculty of Ciraduate Studies and lRevarrh in partial fulfilment of the requirements for the degree of Naster of Enginering

Ottawa-Carleton Institute for Flectrical Finginerring

Department of Electromics

Carleton Iniversity

Ottawa. Canada

(C)1996. Phanilatha Vishmublotiln 
National Library

of Canada

Acquisitions and

Bibliographic Services Branch

395, Weltington stlewt

Orawa Ontaro

KIA ONA4
Bibitotheque nationale

du Canada

Direction des acquisitions et

des services biblographiques

395 rue Wellington

Orawa (Omanks)
The author has granted an irrevocable non-exclusive licence allowing the National Library of Canada to reproduce, loan, distribute or sell copies of his/her thesis by any means and in any form or format, making this thesis available to interested persons.
L'auteur a accordé une licence irrévocable et non exclusive permettant à la Bibliotheque nationale du Canada de reproduire, prêter, distribuer ou vendre des copies de sa thèse de quelque manière et sous quelque forme que ce soit pour mettre des exemplaires de cette thèse à la disposition des personnes intéressées.

L'auteur conserve la propriété du droit d'auteur qui protège sa thèse. Ni la thèse ni des extraits substantiels de celle-ci ne doivent être imprimés ou autrement reproduits sans son autorisation.

ISBN $0-612-19509-0$ 
Phanilatha lishaubhotla

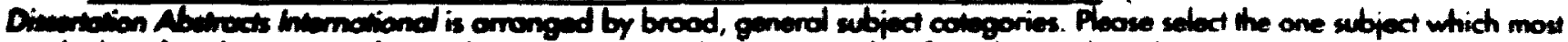
nenty deacribes the coment of your discontotion. Enver the corresponding four digit code in the spoces provided.

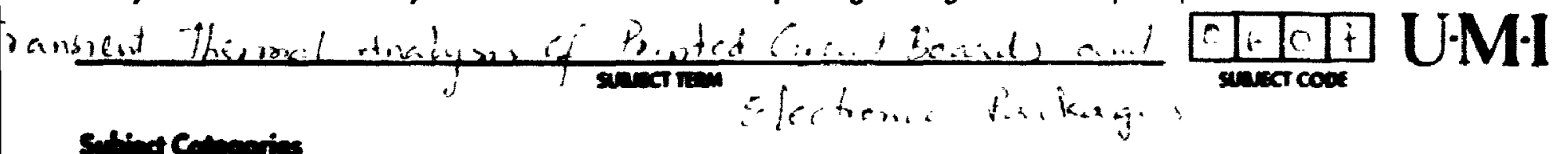

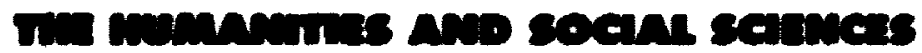
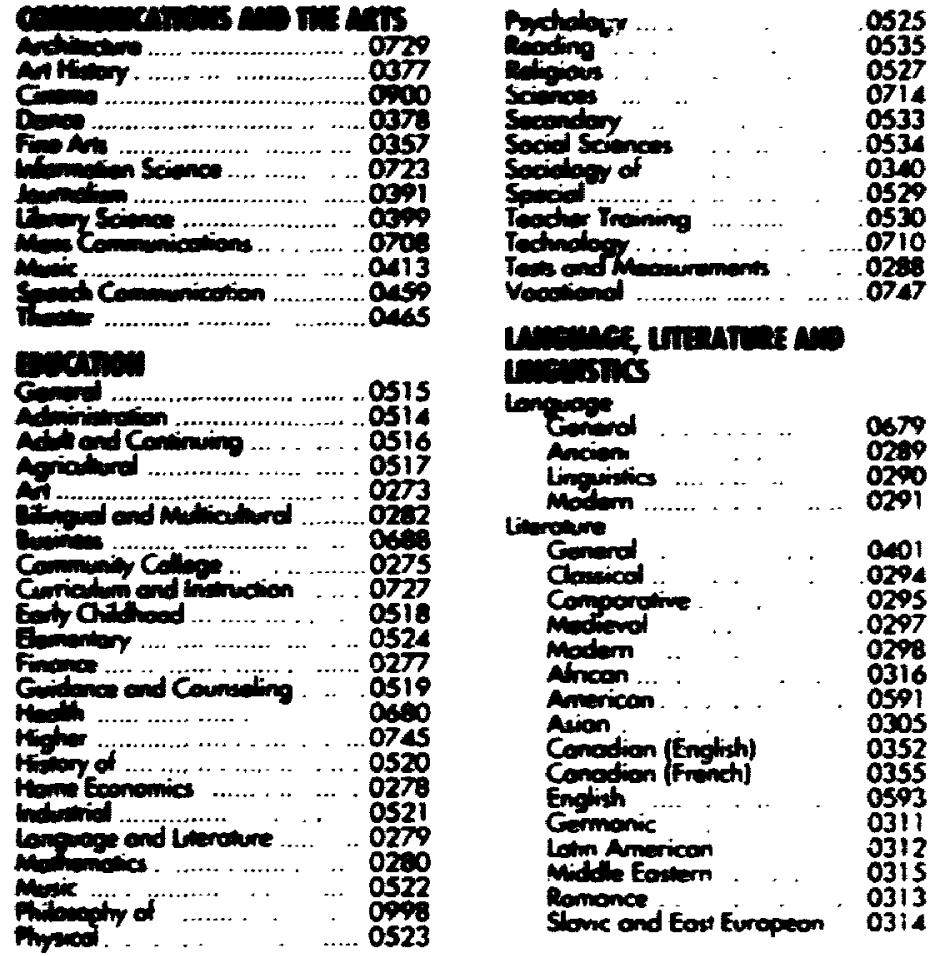

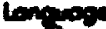

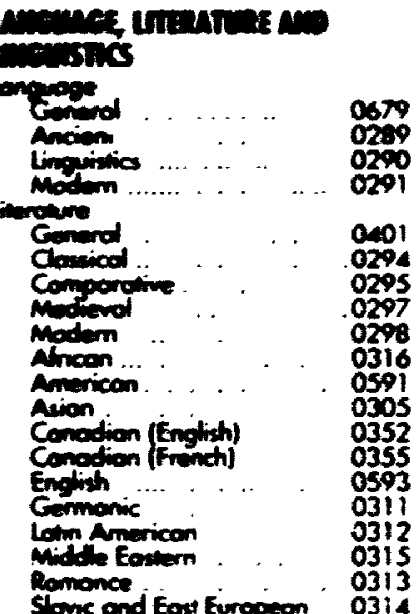

25

27

633

O534

259

0530

0283

11.

0517

20.7.

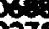

0727

0518

927

osio

0745

0520

0s21

0279

os 22

myex.

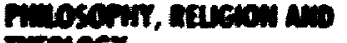
Tiniest

Phouphy

Detion

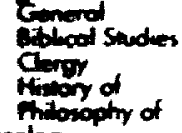

Theology

secustings

Ancrican Stud

Armopolog.

Coinurd

Anmexi:

Business Admunistrotion

Geremel

Acoouning

Bonting

Manogemen

Conoton Sidues

Economics

Generd

Corcuturel

Finonce

Hontony

not

Fallione

Geogrophy

Mrston

Gerol

0372

0373

0300

ali1

0345

orss

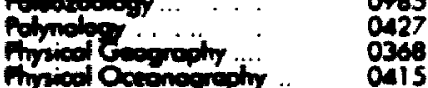

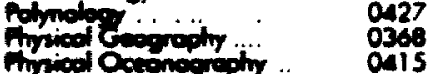

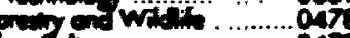

Q

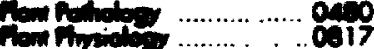

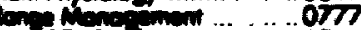

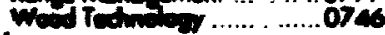

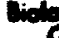

ctimed .................... 0308

Antar ...................027

C. . ..................

c)

cedver ........................0379

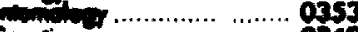

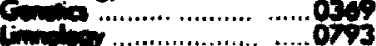

Monot...................... gito

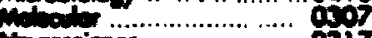

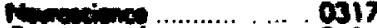

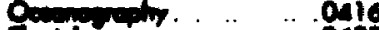

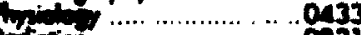

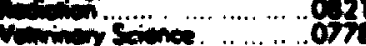

yon?

cinision

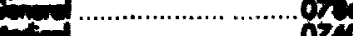

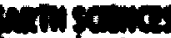

anting

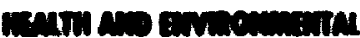

stincs

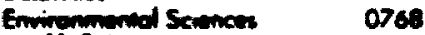

Hecin Sciones

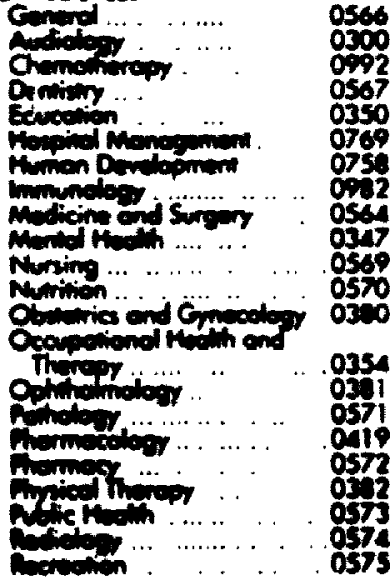

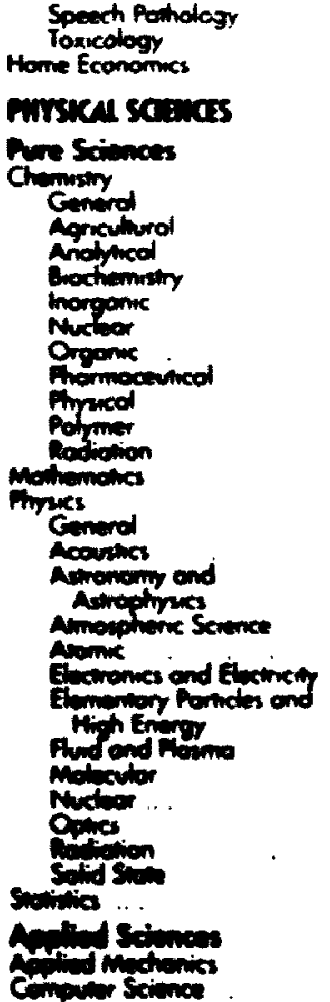

0460

0383

0386

0485

0749

Oabs

aes?

aute

0738

0490

nos

0495

075
0.05

ocos

oino

0005

060

0718

0.07

nros

0759

0009

Osio

075

OPS:

0.63

0346

ove
0422

0318

032

0310

0320

0460

0323

032

0326

032

0310

0272

0770

0338

0385

050 !

0503

0505

0500

0510

OSII

0356

0366

0351

0578

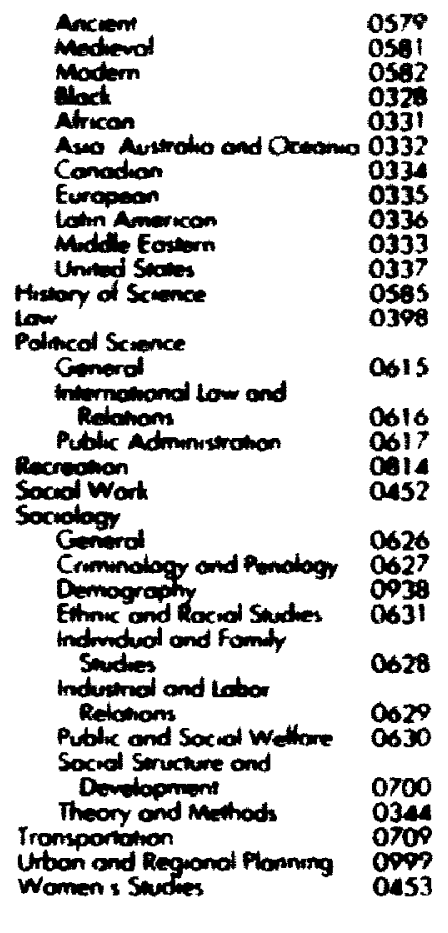

0579

582

33i

334

335

0333

0585

abis

0616

0.14

0626

627

0631

0628

\begin{tabular}{|c|}
\hline 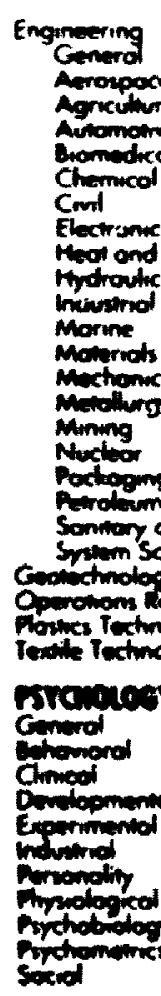 \\
\hline
\end{tabular}

0700

700

0453
0537

0538

0530

OSal

ose 2

osi 3

Osa

oses

$05 e 6$

OSa7

0704

0551

0552

0549

Osse

2470

o795

0 osa

0621

030

$042 ?$

0420

042

025

oves

0332

Qasi 
The undersigned recommend

to the Faculty of Graduate Studies and Research

the acceptance of the thesis

\section{Transient Thermal Analysis of Printed Circuit Boards and Electronic \\ Packages}

submitted by Phanilatha V'ishnubhotla.

in partial fulfilment of the requirements for

the degree of Master of Engineering

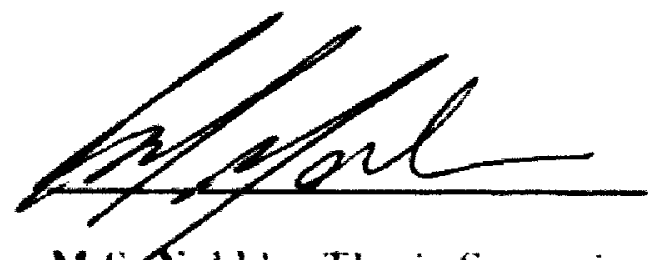

M.S. Saklia. Thesis Suprerisor

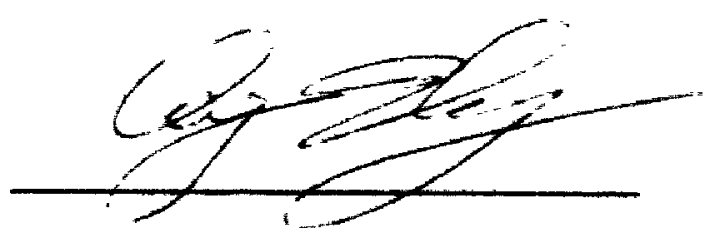

Q.J. Zhang. Thesis Co-Supervisor

Myoutir J.S.ht

J. Wight. Chair. Department of Electronics 


\section{Abstract}

Development of highly integrated and denser semiconductor module configura tions. with greater power consumption rates make thermal design increasingly im. portant. This thesis presents a new solution method for transient thermal analysis of electronic systems. The method is based on combining finite element techunume and efficient moment-matching terhnique originally developed in the rircuit simmlation area for solving large set of linear differential equations. Finite element modeling in space/time domain of a complete electronic system untally result in a large sot of ordinary differential equations. which can be solved using various time sepping algorithms. However. efficiency of ronventional algorithms is linited by their cumpun. tational cost and stability criteria. This thesis presents application of the mement matching technique for solving thermal finite dement equations. Several exatmplen are presented to illustrate the methodolog: A comparison of the propused wivethen with conventional solution ter hnigues shows sulst am ial improwennent in the compunt tional efficiency without sacrifice in accuracy. Also, in this thesis application of cirewit simulation techniques for solving nonlinear transient thermal probleme is presented. 


\section{Acknowledgments}

Firstly. my grateful appreciation is extended to my supervisor Prof. M.S. Nakhla for his encouragement, continued assistance. expert guidance and supervision throughout the course of my graduate studirs and research. My great thanks also go to Prof. Qi-Jun Zhang for his invaluable advice and comments on the research work.

I wish to thank all the staff and fellow graduate students in the Department of Flectronics for their assistance and friendship.

Finally. I wish to take thic opportunity to express my deepest appreciation to my parents and my husband. Without their encouragement and endless support. I would not have the opportunity to complete this work. Last bust not the least I wish to acknowledge my little son for his extended comperation during my studies. 


\section{Contents}

1 Introduction 1

1.1 Motivation .......................

1.2 Organization of the The sir . . . . . . . . . . . .

2 Thermal Modeling of Electronic Systems 6

2.1 Introduction . . . . . . . . . . . . . . . . .

2.2 Heat Transfer: concepts . . . . . . . . . . . . . . . o i

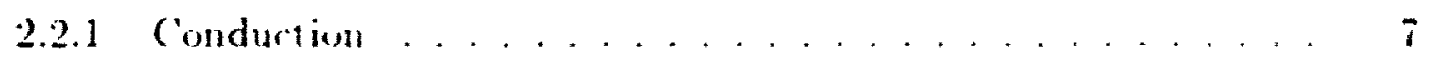

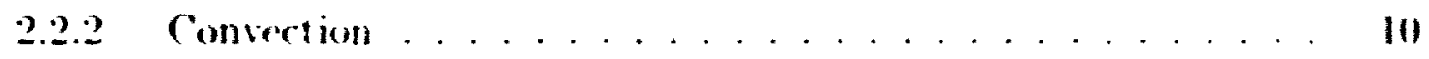

2.2 .3 Radiation ....................... 11

2.3 Thermal Tratsport in Electronir Systems. . . . . . . . . 13

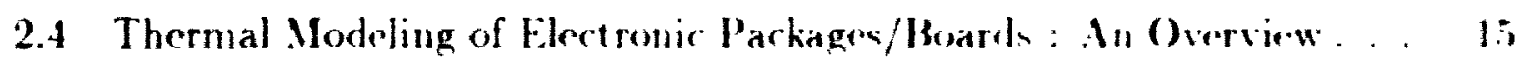

2.4.1 Closed Form Methods . . . . . . . . . . . . . Is

2.4 .2 Thermal Nefwork Method . . . . . . . . . . . 19

2.4.3 Finite Difference Method . . . . . . . . . . . . 20

2.4.t Finite Flement Method . . . . . . . . . . . . 21

2.4.5 Experimental Mrthods . . . . . . . . . . . . . 22

2.4.6 Other Methods . . . . . . . . . . . . . . . 22

3 The Finite Element Method 24 
3.1 Introduction . . . . . . . . . . . . . . . . . . . 24

3.2 The Finite Element Method . . . . . . . . . . . . . . 24

3.2.1 Generalization of The Finite Element Method - Weighted Resid-

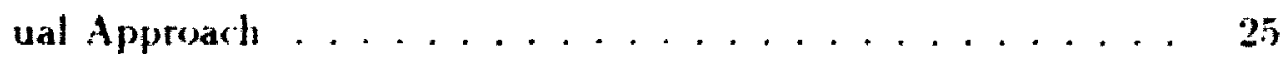

3.2.2 FE.M Applied to Heat Diffusion Problems . . . . . . . . 28

3.3 Overview of Numerical Techniques for Time Domain Analysis . . . . 30

3. Dired Integration Tedhigues ................... 30

3.5 Modal Method . . . . . . . . . . . . . . . . . . . . 33

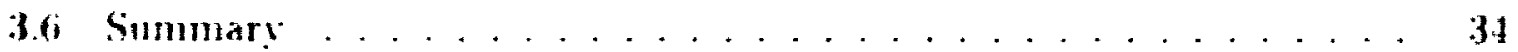

4 Moment Matching Technique $\quad 35$

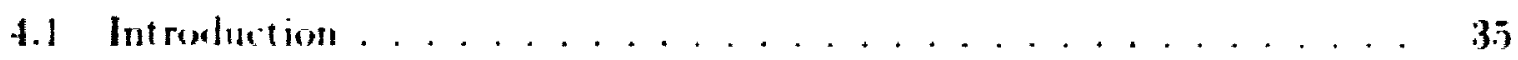

1.2 Momen Matrhing Method . . . . . . . . . . . . . . . . 35

4.2.1 Syotem Moments ...........................

4.2.2 Cenerating an Approximate Response . . . . . . . . . . 3

1.2.3 Pules and Residues . . . . . . . . . . . . . . 39

4.1 Computational Effort for Moment Matching Method . . . . . . . . 40

5 Thermal Modeling Based on FEM and Moment Matching Method 41

5.1 Introduction . . . . . . . . . . . . . . . . . . 41

5.2 Generating the Fectrical Votlist From the Finite Element Matrices . 43

5.2 .1 Example...................... 43

5.3 Sunumary of the Notlist Extrartion Procedure . . . . . . . . . . 45

5.t Examples of Printed Circuit Boards and Packages . . . . . . . . . 46

5.4.1 Fixamplel: Printed Circuit Board with 3 Dual-in-Line Parkages 47

5.4.2 Kxampley: Single Component on Board . . . . . . . . 50 
5.4.3 Example3: Power Transistors on Panel . . . . . . . . . (i)

5.5 Discussion of the Results . . . . . . . . . . . . . . (i. .

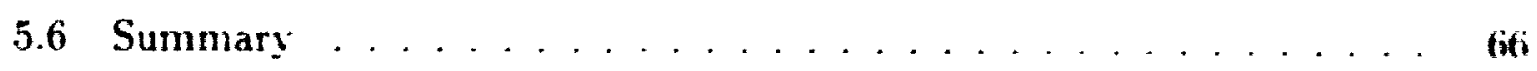

6 Nonlinear Transient Thermal Analysis Using Circuit Simulation Techniques $\quad 67$

6.1 Introdıction . . . . . . . . . . . . . . . . . 6.

6.2 Problem Formulation . . . . . . . . . . . . . . . $6 \mathrm{G}$

6.3 Solution of Network Equations I sing Implicit Integration and NewtonRaphson Method .........................

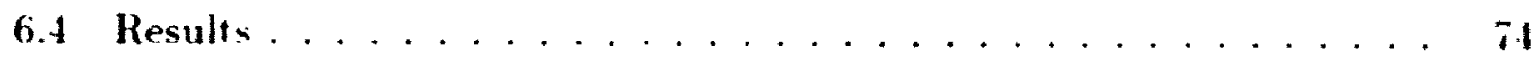

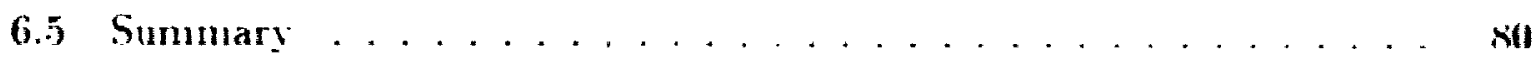

7 Conclusions $\quad \mathbf{8 1}$

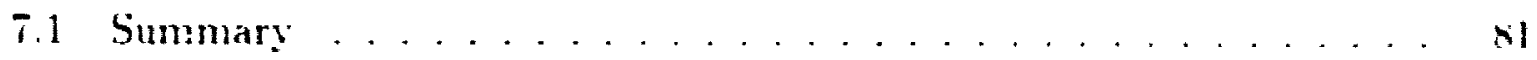

7.2 Scope for Further Work . . . . . . . . . . . . . . . 


\section{List of Figures}

2.1 Leat conduction in one dimensional solid element . . . . . . .

2.2 Volume element used in heat balance . . . . . . . . . . . .

2.3 Heat exchange in one dimension . . . . . . . . . . 11

2.4 Dual-in line package on a $\mathrm{PCB} \ldots \ldots \ldots \ldots$

2.5 Schematic cut-view of a dual-in-line parkage ......... 14

2.6 Lleat flow pathis for a dual-in-line packages mounted on a PCH . . . I6

2.7 Heat flow paths for a power transistor . . . . . . . . . . 17

3.1 Problem domain $\Omega$ and bondary $I \ldots \ldots \ldots \ldots$

3.2 Dincretization in the time domain $\ldots \ldots \ldots . \ldots \ldots$

5.1 Dimensions and boundary conditions ... . . . . . . . . . 4

5.2 Axisymmetric finite element model $\ldots \ldots \ldots \ldots$

5.3 Equivalent electrical circuit representation for $i^{\text {th }}$ row of thermal capacitance matrix C . . . . . . . . . . . 46

5.4 Dual in-line packages on a priated circuit board (Example 1) .... 48

5.5 Finite element discretization of the PC'B in Example $1 \ldots \ldots$

5.6 Transient temperature response (Example 1) . . . . . . . . . . 51

5.7 Comparison of temperature response using moment - matching and implicit integration techniques (Example 1) . . . . . . . . 52 
5.8 A printed circuit board with a surface mount componint (Example:2)

5.9 Axisymmetric finite element model of a $\mathrm{PCB}$ in example?

5.10 Comparison of temperature response using moment -matching and implicit integration techniques for the example in Fig. i.?

5.11 Transient temperature response (Example $21 \ldots \ldots$

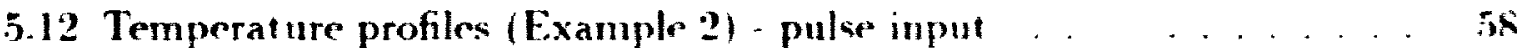

5.13 Transient temperati:re respence (Fxample 2) - pulae input . . 5!

5.14 Power transistons on a pancl . . . . . . . . . . . . . . il

5.15 Power transistors on a panel - Finitre elemont model . . . . . . . . ti:

5.16 Comparison of temperature response using moment matching and im plicit integration techniques for the example in Fig. 5.15 . . . . . (ii)

5.17 Transient temperature response using moment-matching asd implic it integration techniques for the axample in Fig. 5.15 .

6.1 3-dimensional thermal uetwork at mork . . . . . . . . . . . . . 70

6.2 Hybrid circuit pachage geometry [1] . . . . . . . . . . . . .

6.3 Thermal network model showing thermal resistors for the hyl,rid rire nit package (a) Top view (b) Side virw . . . . . . . . . . . T

6.4 Temperature profiles using Newton-Raphson method and implicit in. tegration technique ...................

6.5 Comparion betwern Newton-Raphosu terhingue and relaxation teel

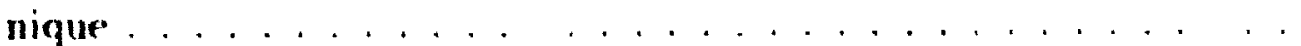




\section{List of Tables}

5.1 Computational statistics and nodal temperature solution at $T=1.500$ (seconds) for the printed circuit board example in Fig. 5.4 . . . . . 50

5.2 Temperature solution at $t=15$ (seconds) for the example in Fig. $5.9 \quad 57$

5.3 Temperature solution at $\tau=1$ (seconds) for the example in Fig. 5.9 pulse input ............................ 60

5.1 Temperature solution for the panel with power transistors . . . . 65

5.5 Number of poles extracted by moment-matching technique . . . . . 65

(i.l Nodal temperature solution for the hybrid package . . . . . . . so 


\section{List of Symbols}

$Q . \quad$ rate of heat flow due to convertion

Q. rate of heat flow due to radiation

$k_{x} \quad$ thermal conductivity in $x$ direction

$k_{y} \quad$ thermal conductivity in $y$ direction

$k$ : thermal conductivity in = direction

4s surface area

h convective heat tramfer coeflicient

$h_{\tau}$ radiative heal iransfer coeflicient

$\mathbf{T}$ temperature vector

T. surface temperature of a body

T) ambient temperature

$\sigma$

Bolt zman const ant

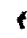

emissivity

$x, y=$ Cartesian coordinales

p density of a material

$c_{p} \quad$ sperific heat

$r$ time

$\hat{q}$ heat generated per unit volume 


\begin{tabular}{|c|c|}
\hline $\boldsymbol{R}_{\boldsymbol{k}}$ & thermal resistance for conduction \\
\hline $\boldsymbol{R}_{\boldsymbol{c}}$ & thermal resistance for convection \\
\hline $\boldsymbol{R}_{\boldsymbol{r}}$ & thermal resistance for radiation \\
\hline$C$ & thermal capacitance \\
\hline $\mathbf{C}$ & capacitance matrix \\
\hline $\mathbf{G}$ & conductance matrix \\
\hline $\mathbf{b}$ & input vector \\
\hline $\mathbf{N}$ & shape functions \\
\hline $\boldsymbol{\Omega}$ & problem domain \\
\hline $\mathbf{\Gamma}$ & problem boundary \\
\hline$\lambda$ & eigenvalue \\
\hline$\phi(\mathrm{T})$ & nonlinear vector \\
\hline 6 & time step \\
\hline$s$ & Laplace operator $(=j \omega)$ \\
\hline $\mathbf{M}_{n}$ & Vector of $n-t h$ moments of a network \\
\hline$m_{n}$ & $n-t h$ moment of an output \\
\hline 7 & Jacobian matrix \\
\hline
\end{tabular}




\section{List of Acronyms and Abbreviations}

DIP

PCB

RC

AWE

BEM

CAD

CPU

TNM

FDM

FEM
Dual-in-Line Package

Printed Circuit Board

Resistance-Capacitance

Asymptotic Waveform Evaluation

Boundary Element Method

Computer-Aided Design

Central Processing Unit, CPU resources connoting computer resources

Thermal Network Method

Finite Difference Method

Finite Element Method 


\section{Chapter 1}

\section{Introduction}

\subsection{Motivation}

The continuous drive to minimize the size of electronic packages while maximizing speed and functionality poses difficulties in designing reliable packages. Present generation packages with increased chip densities and higher volumetric heat generation rates, call for an efficient thermal design of the entire electronic system to achieve optimum electrical performance [2]. This necessitates detailed thermal design of complete electronic systems. which is very time consuming on current computing platforms. The accuracy and the computational time required for the solution are largely dependent on the numerical technique used. The use of simulation techniques in thermal design facilitates proper design of -lectronic structures, consequently reducing the number of experiments required.

Several thermal nnalysis methodologies have evolved over a period of time ranging from closed form and thermal network methods [3] to CPU intensive techniques such as finite element method(FEM). One of the variables considered to be of impor- 
tance in selecting a particular software for thermal design is problem set up time. which encourages easy problem modification for parametric study and execution speed. Closed form and thermal network(TN) based solutions with low problem set up time tend to be appropriate when the problem geometry is simple. Thermal network method(TNM) is also widely used at the preliminary design stage and sometimes at advanced design stages for its simplicity, depending on the accuracy of the results desired.

FEM has become a vital efficient tool for thermomechanical design. First, FEM is capable of mathematically simulating irregular. complex geometries where a rlosedform solution is almost impossible to obtain. Furthermore FEM is especially advantageous in conducting sensitivity analyses since changes in parameters such as material properties etc., can be easily incorporated $[4,5]$.

For thermal analysis of electronic systems using FEM, problems are commoniy formulated in space/time domain. Space/time domain formulation leads to a set of ordipary differential equations, which have to be solved in the time doimain. The size of the equations to be solved is usually large. Usage of conventional solution algorithms is restricted by computing time, since it is often necessary to take very small time step size to avoid undesirable numerically induced oscillations [6, 7$]$. Also, they require solution of large set of algebraic equations at each time point.

A systematic approach of hybrid Laplace transform/finite element method has been proposed $[8,9,10]$ for heat transfer problems. In these applications, the heat conduction equation, which describes the transient temperature distribution in solid domain, together with proper initial/boundary conditions, is transformed to thr Iaplare domain and then solved by conventional node-based finite element method. In $[9]$ sim. ilarity transform method on a matrix of complex coefficients is used to overcome the 
restriction of calculating one nodal solution at a time. An extension of [9] for temperature dependent thermal conductivity problem is given in [10], where perturbation method is used to linearize the nonlinear equation.

In this thesis a new technique is proposed, which falls in the category of $[8,9]$. However, the main difference is that it requires the computation of dominant natural modes only and thus eliminating the major computing cost of determining ail the eigen modes. The new solution technique is based on moment-matching technique, which has been used successfully in circuit simulation area for efficient transient response of large size circuits $[11,12,13]$. It has been shown that this technique can be used to simulate large circuits with negligible error compared to conventional circuit simulation techniques while 2 to 3 orders of magnitude faster. Using momentmatching technique. the Laplace domain transfer function of a large linear network will be approximated by a reduced-order model containing relatively small number of poles. Hence, an attempt is made to take advantage of a) flexibility of FEM in modeling complex body shapes, such as populated p.inted circuit boards, electronic packages, etc., b) accuracy of the moment-matching technique with no time domain discretization to obtain the solution faster. This new technique can overcome the drawback of the stiffness problem encountered in linear transient problems.

In reality, thermal conductivity $k$, of the materials used in electronic packages is a function of temperature. Also, $\frac{\partial k}{\partial T}$ may be positive or negative, depending upon the material and sometimes temperature as well. Similarly, convective heat transfer coefficient, $h$ may depend on temperature. Temperature dependence of $k$ and/or $h$ makes the heat conduction equations nonlinear. Radiation is another important source of nonlinearity.

A procedure for solving nonlinear transient thermal problems using electrical cir- 
cuit simulation techniques is also presented in this thesis. Thermal network method (TNM) is used for the problem formulation where the thermal network is analogous to electrical resistance, capacitance network.

Space and time descritisation is used to replace the nonlinear differential equations by a set of nonlinear algebraic equations. The resulting nonlinear algebraic equations are solved using implicit integration techniques and Newton-Raphson method implemented in a circuit simulator. To illustrate the methodology transient temperature analysis results for an example of a hybrid package are given.

\subsection{Organization of the Thesis}

Chapter 2 defines the thermal transport in electronic systems and presents a brief discussion of basic heat transfer mechanisms. A conceptual overview of the existing thermal modeling methodologies useful in the thermal analysis aspects of electronics packaging is given.

Chapter 3 describes the basic finite element concapts. Application of FFM to solve heat transfer problems is discussed in detail. The steps involved in finite element formulation of heat diffusion equation in the time domain are presented. Various numerical techniques are disc'issed which can be used for the solution of resulting ordinary differential equations.

Chapter 4 describes the concept of moment-matching technique.

the finite element matrices and their transformation to the electrical circuit

Chapter $\mathbf{5}$ describes the steps involved in transforming the finite element mquations to the Laplace domain. The process of extracting the finite element matrices and their transformation to the elect rical circuit elements using the software provided 
by EMRC $[14,15,16]$, is also discussed.

Using the above procedure transient temperature results for examples of printed circuit boards and packages are presented. Comparison of accuracy and computational efficiency of the proposed technique is made with the implicit integration techniques.

Chapter 6 describes the thermal simulation methodology using the thermal network method and the solution technique details. Nonlinear temperature results are given for a hybrid package example. Results are compared using the solution technique outlined and commonly used relaxation methods.

Chapier 7 gives a summary of the thesis and suggestions for further work. 


\section{Chapter 2}

\section{Thermal Modeling of Electronic Systems}

\subsection{Introduction}

In his chapter basic heat transfer concepts and thermal transport phenomena in electronic systems are discussed. Some of the solution methods for thermal analysis of electronic systems are discussed and the literature reviewed, providing a setting for the work presented in the later chapters.

\subsection{Heat Transfer: Concepts}

The heat transfer processes can be categorized into three basic mechanisms or modes, which are conduction, convection and radiation [17]. Practical problems will involve at least two of the possible modes and sometimes all the three may be involved simultaneously. 


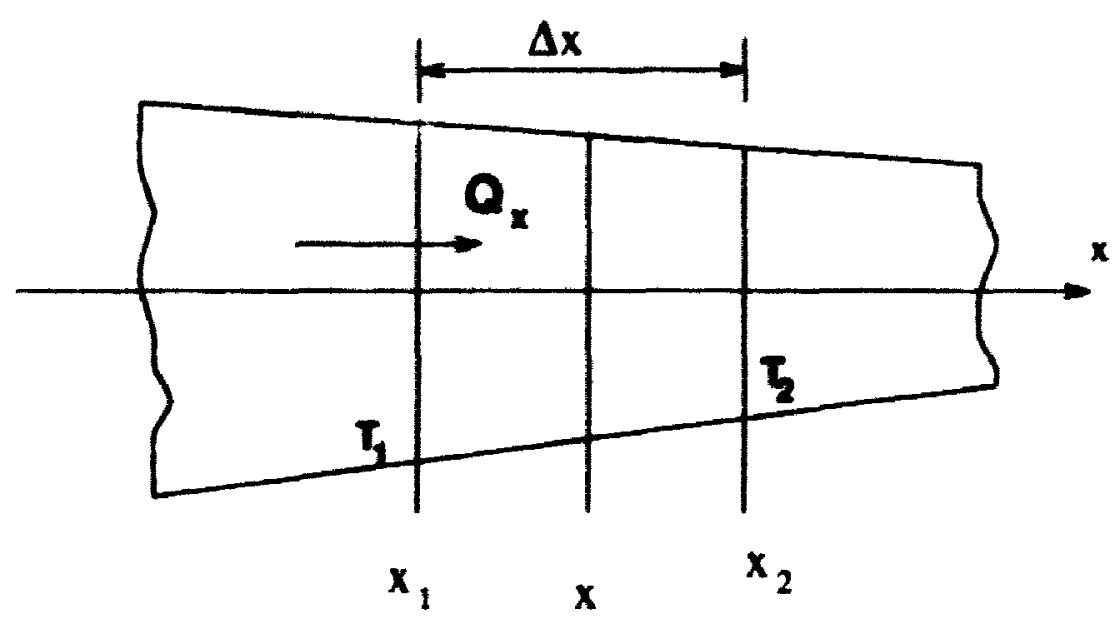

Figure 2.1: Heat conduction in one dimensional solid element

\subsubsection{Conduction}

Conduction is the process of heat transfer which involves internal heat energy exchange from one body to the other. The flow of energy is from a body at a higher temperat ure to a body at lower temperat ure. The dist inguishing feature of conduction is that it takes place within the boundaries of a body or across the boundary of a body placed in contact with the other. The basic law governing the heat conduction process is Fourier's law. Considering heat conduction through a solid body in one dimension as shown in Figure 2.1. Fourier's law can be represented as.

$$
\begin{gathered}
Q_{k}=k \cdot A_{k} \frac{\left(T_{1}-T_{2}\right)}{\Delta x} \text { or } \\
Q_{k}=-\left.k A_{k} \frac{d T}{d x}\right|_{x}
\end{gathered}
$$

where $Q_{k}$ is the rate of heat flow due to conduction across a body of thickness I.r, with a cross sectional area of $A_{k}$ and thermal conductivity $k$. $T_{1}$ and $T_{2}$ are the 

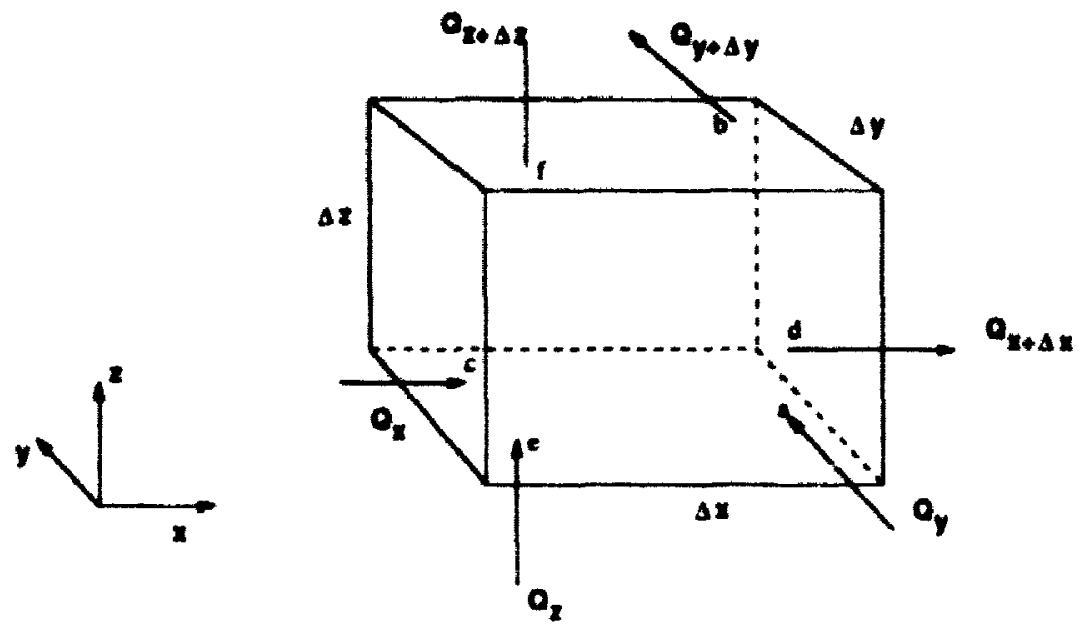

Figure 2.2: Volume element used in heat balance

temperatures at the two ends of the body: The thermal resist ance, $R_{k}$, for conduction can be writtert as.

$$
R_{k}=\frac{\Delta x}{k \cdot A_{k}}
$$

Generalization of Fourier's law in three dimension can le accomplished by ap. plying the one dimensional law to an element of volume $\Delta I^{\circ}=\Delta x \Delta y \Delta z$ as shown in Figure 2.2 for which

$$
\begin{aligned}
Q_{x} & =-k_{x} \Delta y \Delta z \frac{\partial T}{\partial x} \cdots \text { at c } \\
Q_{x+\Delta x} & =-k_{x} \Delta y \Delta z \frac{\partial T}{\partial r} \cdots \text { at d } \\
Q_{y} & =-k_{y} \Delta x \Delta z \frac{\partial T}{\partial y} \cdots \text { at a } \\
Q_{y+\Delta_{y}} & =-k_{y} \Delta x \Delta z \frac{\partial T}{\partial y} \cdots \text { at b } \\
Q_{z} & =-k_{z} \Delta_{y} \Delta x \frac{\partial T}{\partial z} \cdots \text { at } ;
\end{aligned}
$$




$$
Q_{z+\Delta z}=-k_{z} \Delta y \Delta x \frac{\partial T}{\partial z} \cdots \text { at } f
$$

where $k_{x}, k_{y}, k_{z}$ are thermal conductivities in $x . y$ and $z$ directions. Applying the conservation of energy principle for the steady state condition. i.e.. balancing the heat in the element to the heat going out of the element. with internal heat generating source $\dot{q}$ Watts/unit volume for the elemental volume $\Delta t^{*}$ results in.

$$
\begin{aligned}
& {\left[\left.\left(-k_{x} \Delta y \Delta=\frac{\partial T}{\partial x}\right)\right|_{c}-\left.\left(-k_{x} \Delta_{y} \Delta=\frac{\partial T}{\partial x}\right)\right|_{d}\right]} \\
& +\left[\left.\left(-k_{y} \Delta r \Delta z \frac{\partial T}{\partial y}\right)\right|_{a}-\left.\left(-k_{y} \Delta r \Delta=\frac{\partial T}{\partial y}\right)\right|_{b}\right] \\
& +\left[\left(-k_{:} \Delta x \Delta y \frac{\partial T}{\partial z}\right) \mid,-\left(-k_{i} \Delta x \Delta y \frac{\partial T}{\partial z}\right) \|_{f}\right] \\
& +\dot{q} \Delta x \Delta y \Delta z=0
\end{aligned}
$$

Dividing (2.4) hy $\Delta x \Delta y \Delta z$.

$$
\left.\left(k_{x} \frac{1}{\Delta x} \frac{\partial T}{\partial x}\right)\right|_{i} ^{d}+\left.\left(k_{y} \frac{1}{\Delta y} \frac{\partial T}{\partial y}\right)\right|_{a} ^{b}+\left.\left(k_{z} \frac{1}{\Delta z} \frac{\partial T}{\partial z}\right)\right|_{e} ^{f}+\dot{q}=0
$$

Taking the limit of $د l \rightarrow 0$ and using

$$
\left.\lim _{\Delta x \rightarrow 0}\left(k_{x} \frac{1}{\Delta x} \frac{\partial T}{\partial x}\right)\right|_{c} ^{d}=\frac{\partial T}{\partial x}\left(k_{x} \frac{\partial T}{\partial x}\right)
$$

the three dimensional steady state heat conduction equation is.

$$
\frac{\partial T}{\partial x}\left(k_{x} \frac{\partial T}{\partial x}\right)+\frac{\partial T}{\partial y}\left(k_{y} \frac{\partial T}{\partial y}\right)+\frac{\partial T}{\partial z}\left(k_{z} \frac{\partial T}{\partial z}\right)+\dot{q}=0
$$

For time dependent problems the steady state energy balance will be modified to account for energy storage or discharge. Which is the differential form of heat stored $\rho c_{p} \frac{a T}{\partial t}$ for the volume element $\Delta l$ of density $\rho$ and specific heat $C_{p}$. Where $\tau$ rep. resents the time. Thus the partial differential equation for time dependent heat conduction is: 


$$
\frac{\partial T}{\partial x}\left(k_{z} \frac{\partial T}{\partial x}\right)+\frac{\partial T}{\partial y}\left(k_{y} \frac{\partial T}{\partial y}\right)+\frac{\partial T}{\partial z}\left(k_{z} \frac{\partial T}{\partial z}\right)+\hat{q}=\rho c_{r} \frac{\partial T}{\partial r}
$$

\subsubsection{Convection}

Convertion is the term applied to the heat transfer mechanisu which occurs in a fluid due to mixing portions of the fluid as a result of gross moventents of the mass of the fluid. The actual process of energy transfer from one fluid particle to the onluet is still one of conduction. but the energy may be transferred from one print in space to another by the displacement of the fuid.

When the fluid motion is set by the difference in densities crealed by temperature differences. the process is termed as natural convection. When the flud motion is caused by external mechanisms (ex: faul pump. ele) the process is called fored convection.

Newton's law governs the convection process which an be written as.

$$
Q_{r}=h A_{s}\left(T_{s}-T_{j}\right)
$$

where $Q_{r}$ is the rate of heat flow from a surface at temperature $\%$, to the sur rounding fluid at a reference temperature $T_{j} . A_{s}$ is the surface aren of convection. $h$ is the heat transfer coefficient or film coefficient, which is a complex function of the physical properties of the fluid, geometry of the solid surface etr... The thermal resistance, $R_{c}$. for convection may be defined as,

$$
R_{r}=\frac{1}{h \cdot 1}
$$

Convective heat transfer has been ronsidered only as onf of the types of homindary conditions to be applied at the surface of a rondurting solid. For example whrn a 

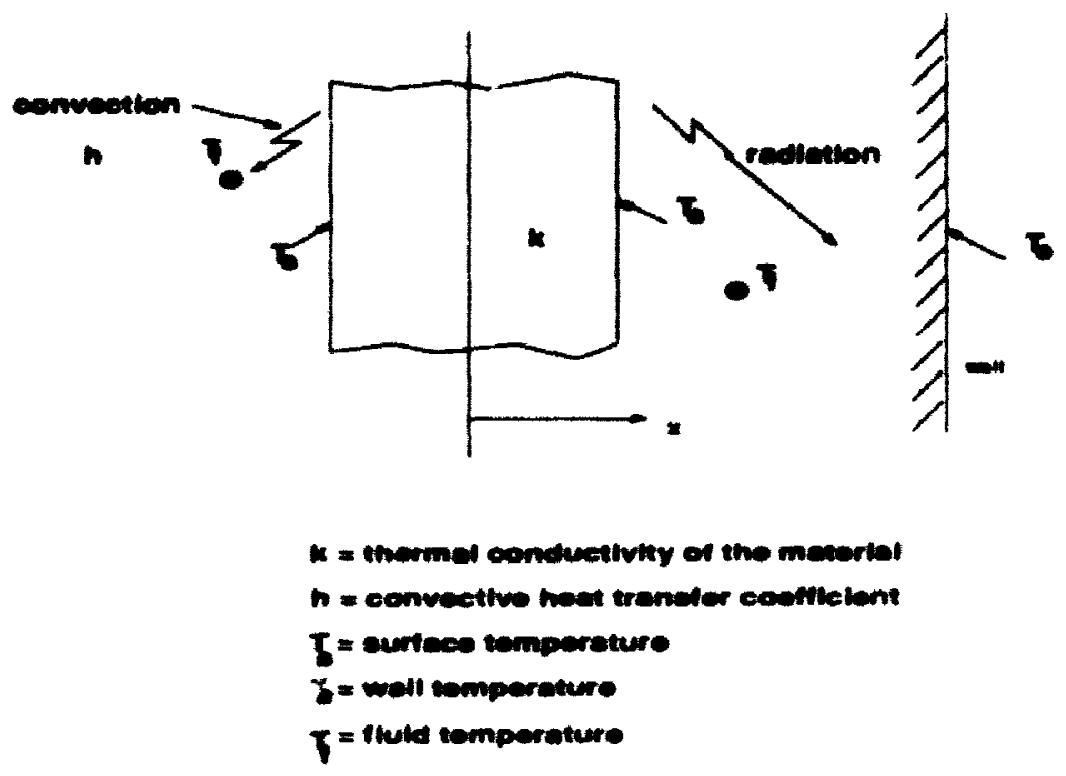

Figure 2.3: Heat exchange in one dimension

condurting body as shown in Figure 2.3 is exchanging heat at its surface with a fluid of specified temperature through a known heat transfer coefficient h. the boundary condition at the surface becomes.

$$
-\left.k\left(\frac{d T}{d x}\right)\right|_{s}=\left.h\left(T_{s}-T_{j}\right)\right|_{s}
$$

\subsubsection{Radiation}

Thermal radiation is the term used to describe electromagnetic radiation which is emitted at the surface of a thermally excited body. This electromagnetic radiation is emitted in all direcions: and when it strikes another body, part of it may be reflected. transmitted or absorbed. The main feature of radiative heat transfer is, the absence of a medium of transport. which is required by conduction and convection processes.

The basic law of thermal radiation is the energy emitted by an ideal black body. 
as given by Stefan-Boltzman equation [17]

$$
Q_{r}=. . \sigma T^{4}
$$

where $Q$ is the rate of radiant emission. $A_{3}$ is the surface area of the body. $T$ is the absolute temperature of the body and $\sigma$ is the Stefan-Boltzman constant.

Radiant heat exchange between a surface at temperatures $T$, and the surronnding environment at temperature $T_{\text {e }}$ can be writtell as

$$
\begin{aligned}
Q_{r} & =A_{s}\left(\sigma\left(T_{s}^{4}-T_{*}^{4}\right)\right. \\
& =\operatorname{ar} \cdot A_{s}\left(T_{s}^{3}+T_{s}^{2} T_{t}+T_{s} T_{*}^{2}+T_{c}^{3}\right)\left(T_{s}-T_{*}\right) \\
& =h_{r} \cdot A_{s}\left(T_{s}-T_{t}\right)
\end{aligned}
$$

where $t$ is the emissivity The temperature dependent radiative heat transfer coefficient. $h_{r}$. is

$$
h_{\tau}=\left(T_{s}^{3}+T_{s}^{2} T_{r}+T_{,} T_{s}^{2}+T_{s}^{*}\right)\left(T_{s}-T_{s}\right)
$$

The thermal resistance, $R_{r}$, for radiation with the forgoing approxination is

$$
R_{r}=\frac{1}{h_{r} A_{s}}
$$

Referring to Figure 2.3. application of radiative boundary conditions at the wurface results in

$$
-\left.k\left(\frac{d T}{d x}\right)\right|_{s}=\cos \left(T_{s}^{4}-T^{4}\right)
$$




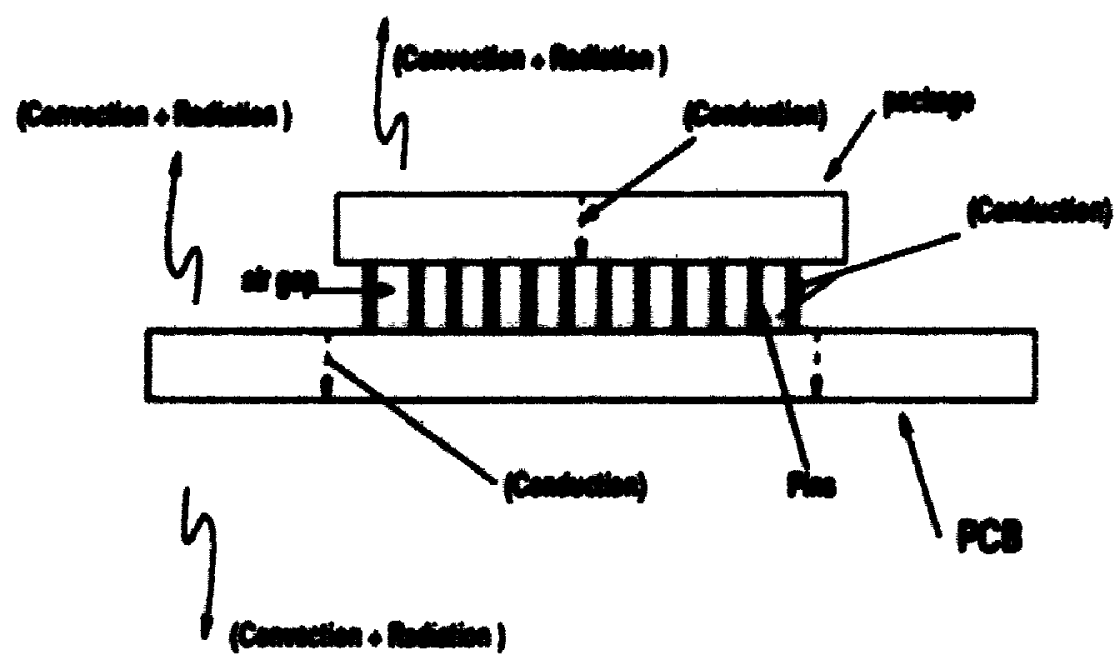

Figure 2.4: Dual-in-line package on a PCB

\subsection{Thermal Transport in Electronic Systems}

An example of a dual-in-line package (DIP) mounted on a PCB, as shown in Figure 2.4 is used to illustrate thermal transport phenomena based on the heat flow mechanisms explained in earlier sections. Details of the DIP assembly are given in Figure 2.5. The heat transfer process for the above configuration includes: heat flow from the package to the PCB via conduction through the pins and the air gap also, via convection and radiation to the ambient. From the top layer of the $\mathrm{PCB}$ to the bottom layer the heat flow is due to conduction. At the boundaries of the PCB i.e, at the top and bottom surfaces, heat is transferred to the surrounding air through the process of convection and radiation.

Using the electrical analogue the above process can be represented as shown in Figure 2.6. The resistances indicate temperature rise per watt $\left({ }^{\circ} \mathrm{C} / W\right)$ of heat transferred between two temperatures. The heat is conducted from the chip junction(s) to the chip package mounting surface through $\boldsymbol{R}_{\text {chip-case }}$, which is the overall thermal 


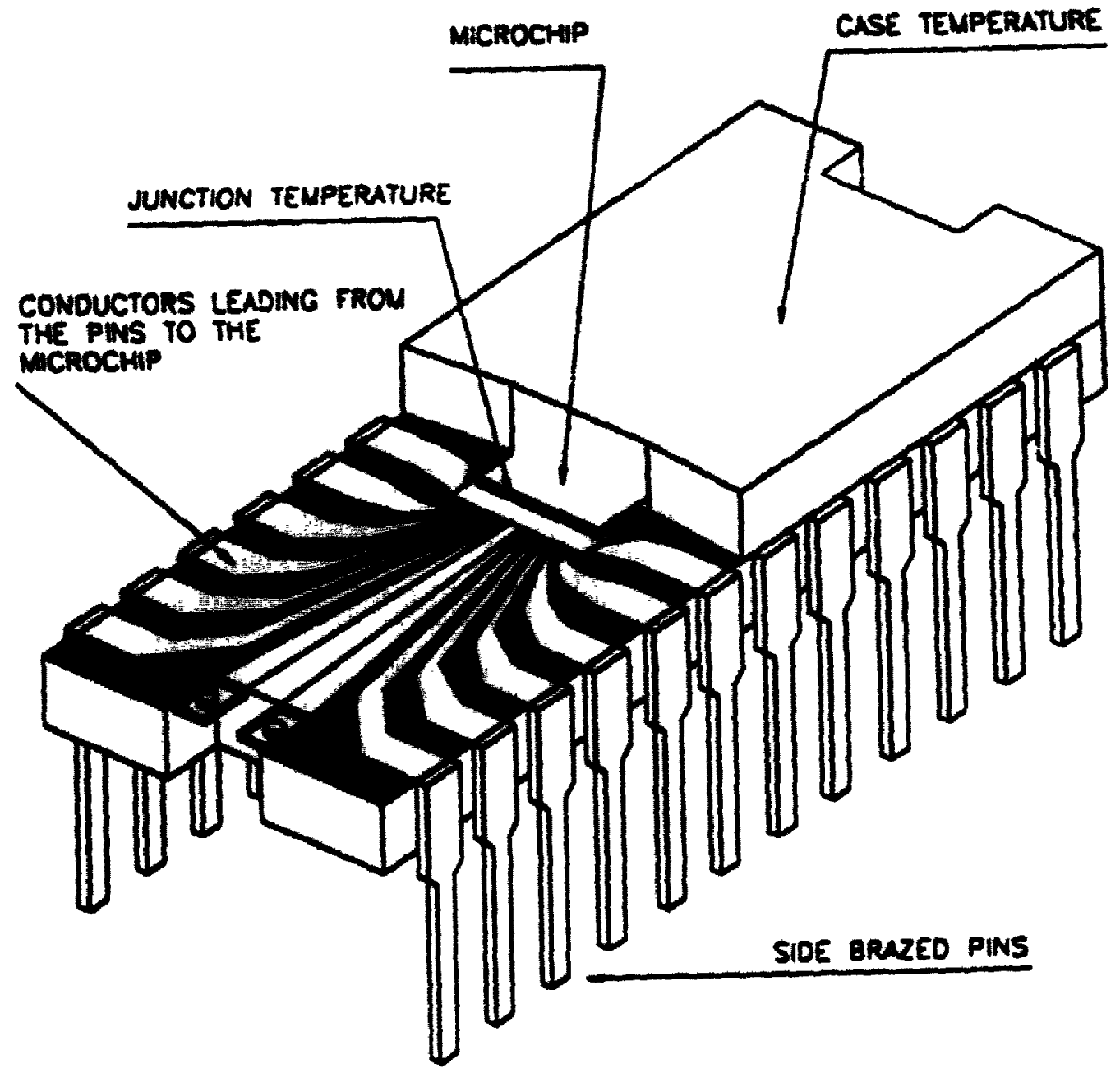

Figure 2.5: Schematic cut-view of a dual-in-line package 
resiatance for heat conduction through the semiconductor chip and chip bonding material. Heal tranofer from the package is distributed between a direct path, $\boldsymbol{R}_{\text {case-air }}$ to the surrounding ambient from the package surface and less direct path to the ambient that involves the circuit board. The path from the package to board can be vis conduction through an air gap element, $R_{\text {pop, }}$ and conduction through the element $\boldsymbol{R}_{\text {,imo, }}$ for the package pins which are often soldered into the board. The board then transmits heat to the surrounding ambient via $\boldsymbol{R}_{\text {board-air. Both }} \boldsymbol{R}_{\text {cese-air }}$ and

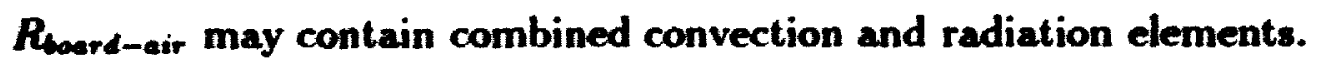

Power transistor packages present a different heat transfer situation where the package base is the major heat transfer surface and is often mounted directly on to a finned heat sink. The electrothermal analogue for a power transistor is shown in Figure 2.7. A resistance $\boldsymbol{R}_{\text {chip-case, }}$, represents both die and the die-package interface resistance. $R_{\text {case-sink }}$ is the resistance between the package and heat sink mounting conditions. $\boldsymbol{R}_{\text {sink-air may represent resistance due to enclosure panel or finned }}$ extrusion.

\subsection{Thermal Modeling of Electronic Packages/Boards : An Overview}

The best way to solve the heat conduction equation $(2.8)$ is to solve analytically. However, there are many situations where the analytical solution is difficult to obtain. For example: a PCB with several chips mounted on its surface is a complex geometry to deacribe mathematically. If the PCB is multi layered and it is made up of different materials it would be mathematically difficult to describe. Problems involving varying material properties in different directions are usually difficult to solve analytically, as 

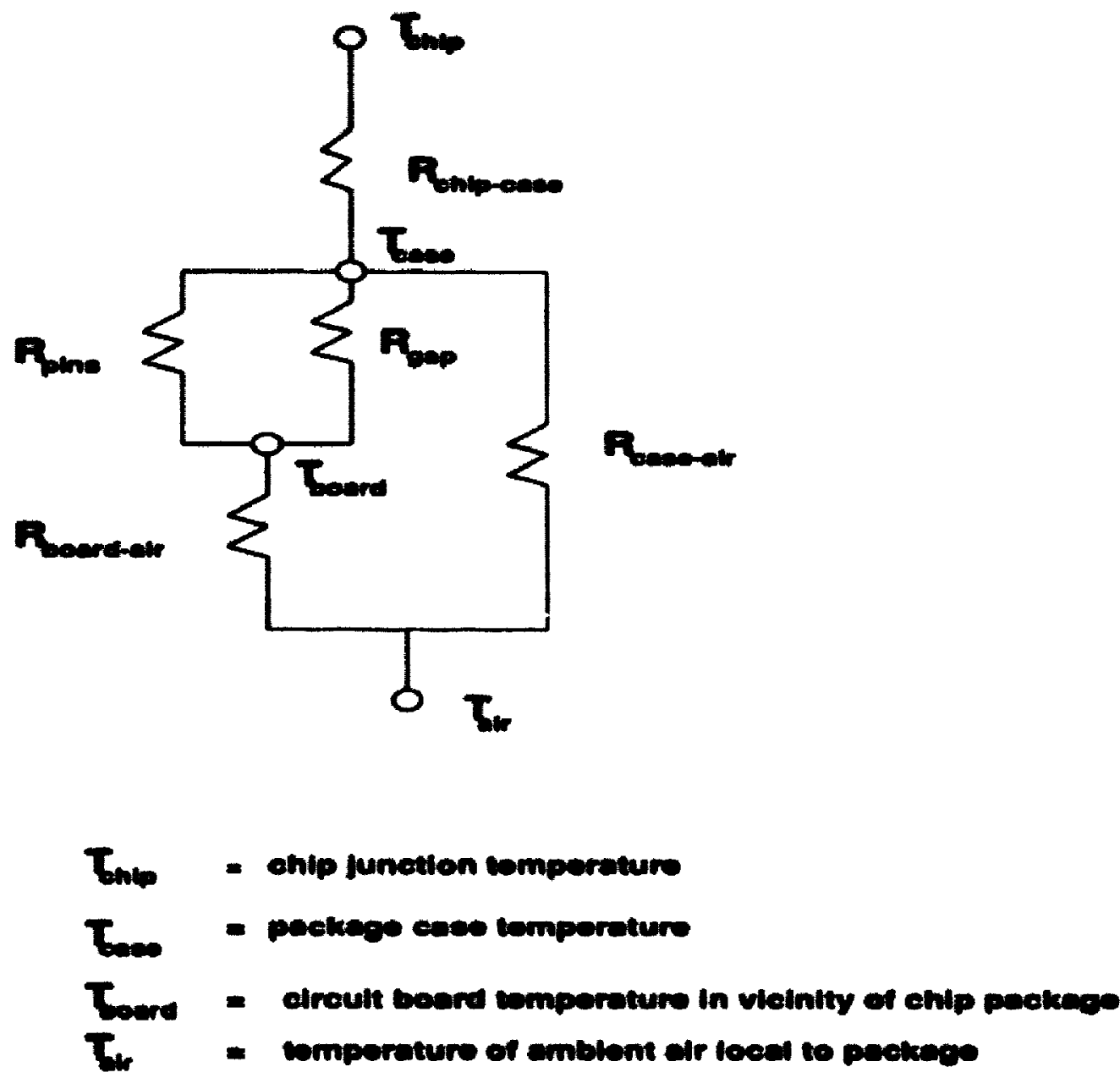

Figure 2.6: Heat flow paths for a dual-in-line packages mounted on a PCB 


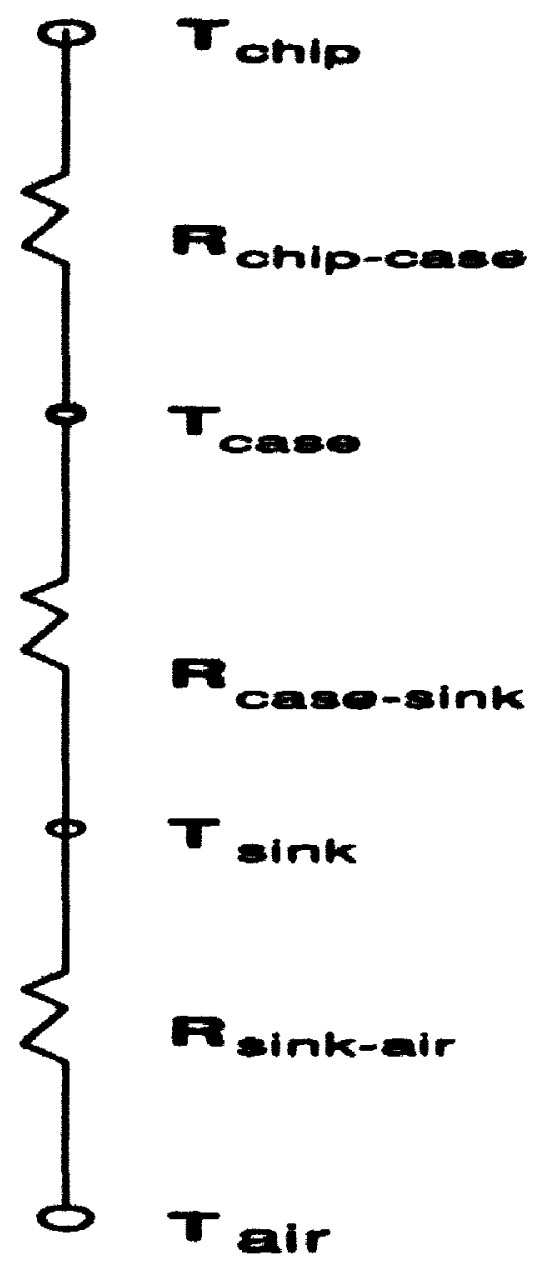

Figure 2.7: Heat flow paths for a power transistor 
are equations having nonlinear terms.

A numerical method can be used to obtain approximate solution when an analytical solution cannot be developed. All numerical solutions produce values at discrete points for one set of independent parameters. The numerical discretization of a continuum problem (2.8) with its associated boundary conditions is the essence of most numerical solutions. The boundary conditions for the heat conduction problem are: 1) essential or Dirichlet boundary condition which is prescription of temperature on part or all of the surface. 2) nonessential or Neumann boundary condition which is prescription of heat flux on part or all of the surface. Convective and radiative boundary conditions are applied when the heat is transferred from the surface to the surroundings through the process of convection and radiation.

\subsubsection{Closed Form Methods}

General heat transfer texts discuss closed form methods [17, 18]. However, a class of solutions using rather standard Fourier series or integral approach have evolved which are useful in solving problems involving cooling of electronics components. Thermal analyzer for multilayer structures TAMS [1] uses the Fourier series approach for thermal modeling of electronic systems. A method is described in [19] which combines the Fourier series solution and lumped thermal resistances to incorpotate the thermal interface resistance between a chip package and board as well as between the same package and ambient. Steady state temperature results are also given for a few examples.

The principal advantage of the closed form solution methods, when applicable, is that geometry meshing is not required. Moreover, they are invaluable for providing benchmark solutions when evaluating a new finite element or thermal network rode. 
The closed form solutions may use different solution techniques, but the specific boundary conditions set applied may or may not be appropriate to the user for solving a variety of practical problems.

\subsubsection{Thermal Network Method}

The thermal network method is one of the widely used techniques for thermal analysis of electronic systems. One of the commercially available software using this technique is TNETFA [1]. This method has the advantage i.e: it does not require exact geometric boundary specifications and the modeling is some what intuitive. The disadvantage is that the effort required to model complex geometries can be extensive. Graphic presentation suffers because of the absence of exact geometric definition data to combine with the results.

The thermal network is essentially a thermal resistance-capacitance (RC) network analogous to electrical $\mathrm{RC}$ network. The resistances are $\boldsymbol{R}_{k}, \boldsymbol{R}_{c}$ and $\boldsymbol{R}_{r}$ for conduction, convection and radiation respectively. $C$ is the thermal capacitance. For simplicity these elements are modeled as linear elements however, in practice they exhibit nonlinear behavior. The nonlinearity is due to the dependency of convective heat transfer coefficient and thermal conductivity of the material on temperature.

The governing equations of the thermal network can be derived using the conservation of thermal energy principle, which essentially balances the heat flowing into the node $i$ with the heat going out of the node. The energy balance equation may be written in different forms depending on the solution method used.

Using a resistor-capacitor network to solve for temperature distribution over printed circuit board packages is discussed in $[20,21]$. Such a RC network model offers aniversal representation of a chip package or rhips on $\mathrm{PCB}$, which is independent of 
the package attachment and cooling configuration [21]. Electrical analogy of thermal network has been used to predict junction temperature and has been verified by measured results $[19,22]$.

Vogelsong and Brzezinski [23] used SPICE, an electrical circuit simulation tool to predict the temperature behavior of electronic devices which allows the sinultaneous simulation of the electrical system and the thermal network. In [24] SPICE was used for thermal and vibration analysis of PCBs. Hefner and Blackburn [25] presented a procedure for developing thermal component models for electrothermal network simulation for incorporating into thermal component libraries of an advanced network simulation program (SABER). Elertrothermal simulation of integrated circuits(ICs) is presented in [26], for simulating transient thermal characteristics of ICs using efficient. macromodeling method based on asymptotic waveform evaluation (AWE). In [27] AWE was used for transient thermal analysis of electronic boards and packages, using TNM for problem formulation.

\subsubsection{Finite Difference Method}

The finite difference method (FDM) approximates the derivatives in the governing differential equations by using central difference equations. This method is useful for solving heat transfer and fluid mechanics problems and works well for two dimensional regions with boundaries parallel to the coordinate axes. The method however is rather cumbersome when regions have curved or irregular boundaries. The finite difference method and thermal network methods are nearly identical as the FDM results in a set of equations identical to those of the thermal net work methorl [2]. 


\section{4.4 Finite Element Method}

The finite element technique is the most powerful and commonly used method for modeling complex body geometries. The finite element method is a numerical procedure for solving physical problems governed by a differential equation. The two characteristics that distinguish it from the other numerical procedures are (1) the method utilizes an integral formulation to generate a system of algebraic equations (2) the method uses continuous piecewise smooth functions for approximating the unknown quantity or quantities. Basic concepts and details of numerical discretization of heat diffusion equation using FEM are given in Chapter 3.

Finite element thermal analysis of direct chip mount electronic systems is given in [28]. Correlation between the model and experimental data is provided and guidance on which direct mount systems can be adequately modeled is described. A decoupling technique, useful in thermal modeling of different PCB and chip configurations is given in [29]. The technique outlined is useful in determining optimal PCB chip configurations reducing the time required for repetitive modeling of the various configurations. FEM code was used to incorporate the controlling algorithm. Parametric study of plastic VLSI packages using FEM is presented in [30]. The thermal stresses induced during the die attach and encapsulation fabrication steps were studied by varying package design parameters such: as die thickness, die bond materials and thickness, lead frame thickness, and package thickness, width and length. In [31] thermal analysis of ceramic pin grid array and multichip module have been carried out using FEM. The effects of air flow, and the package design parameters [30] on the internal and external resistances of the package are also given. 


\subsubsection{Experimental Methods}

Experimental methods play an important role in all aspects of heat transfer. Correlation of experimental and analytical results is an important sspect for modifying the analytical model judiciously. Experimental calculation of the critical parameters such as heat transfer coefficient for components on printed circuit boards is discussed in [32]. The effect of component geometry and layout on the flow distribution is given in [33]. It is concluded that the location and orientation of components with large aspect ratio significantly affects the circuit pack flow distribution. Two techniques for transient temperature measurement are given in [34]. One of the techniques employs a change in the optical index due to temperature variations, which would produce large difference in optical signals. The second method utilizes the thermoelectric effert of an electric resistance for transient temperature measurement.

\subsubsection{Other Methods}

Funk et.al. [35] described semi-analytical method to predict the steady state temperatures on PCBs subjected to single or multiple heat sources. The temperature solution for the package was determined using an iterative procedure between the chip model and the board model. Analytical solution for the temperature field arising from the application of a source heat on an adiabatic plate or board under forced air cooling conditions is discussed in [36]. Temperature solution for multiple sources is also discussed. Transient temperature solution of a two layer semi-infinite plate structure with embedded heat sources was obtained using a three dimensional analytical model in [37]. The solution was obtained for a variety of device structures.

The boundary element method (BEM) is an alternative to FEM. BEM can be 
applied to bounded or unbounded domains. Like FEM, BEM uses nodes and elements, but only on the boundary [38]. Thus as compared with FEM dimensionality is reduced by one. With an increase in the ratio of surface to volume, BEM becomes a less attractive alternative to FEM, because a mesh must be supplied for each boundary. 


\section{Chapter 3}

\section{The Finite Element Method}

\subsection{Introduction}

In this chapter, the general finite element concepts are discussed considering weighted residual approach for the problem formulation. These concepts will be used for the finite element formulation of the heat diffusion problem. The steps involved in obtaining a set of linear algebraic equations, starting from the partial differential equation representing three dimensional heat conduction in a solid are presented. An overview of various numerical techniques used for solving transient heat transfer problems is also presented.

\subsection{The Finite Element Method}

Finite element method (FEM) is a numerical procedure for analyzing structures and continua, usually for problems which are too complicated to be solved satisfartorily by classical analytical methods. The problems may be in the fields of heat conduction, electro magnetics, stress analysis etc. The finite element procedure produces many simultaneous algebraic equations which can be solved using numerical techniques. 
FEM can be defined as a method of approximation to continuum problems such that

(a) the continuum is divided into a finite number of parts (elements), the behavior of which is specified by a finite number of parameters and

(b) the solution of the complete system as an assembly of its elements follows precisely the same rules as those applicable to standard disc ete problems [39].

A finite element analysis typically involves the following steps.

(1) Dividing the structure into finite elements. Mesh generation programs, called preprocessors are used at this stage.

(2) Formulating the properties of each element. Which means, determining the nodal heat fluxes associated with all element temperature fields that are allowed.

(3) Assembling elements to obtain the FE model of the structure.

(4) Applying known nodal heat fluxes.

(5) Solving the simultaneous linear algebraic equations to determine the nodal temperatures.

(6) Calculating the nodal heat fluxes from the nodal and element temperature field interpolations. At this stage a post processor can be used to interpret the results graphically.

\subsubsection{Generalization of The Finite Element Method - Weighted Residual Approach}

Many continuum problems arise in engineering and physics and usually these problems can be represented by appropriate differential equations. Boundary conditions are applied to the unknown function or functions for the solution.

Writing the problem to be solved in a most general fashion, assuming ' $u$ ' as 


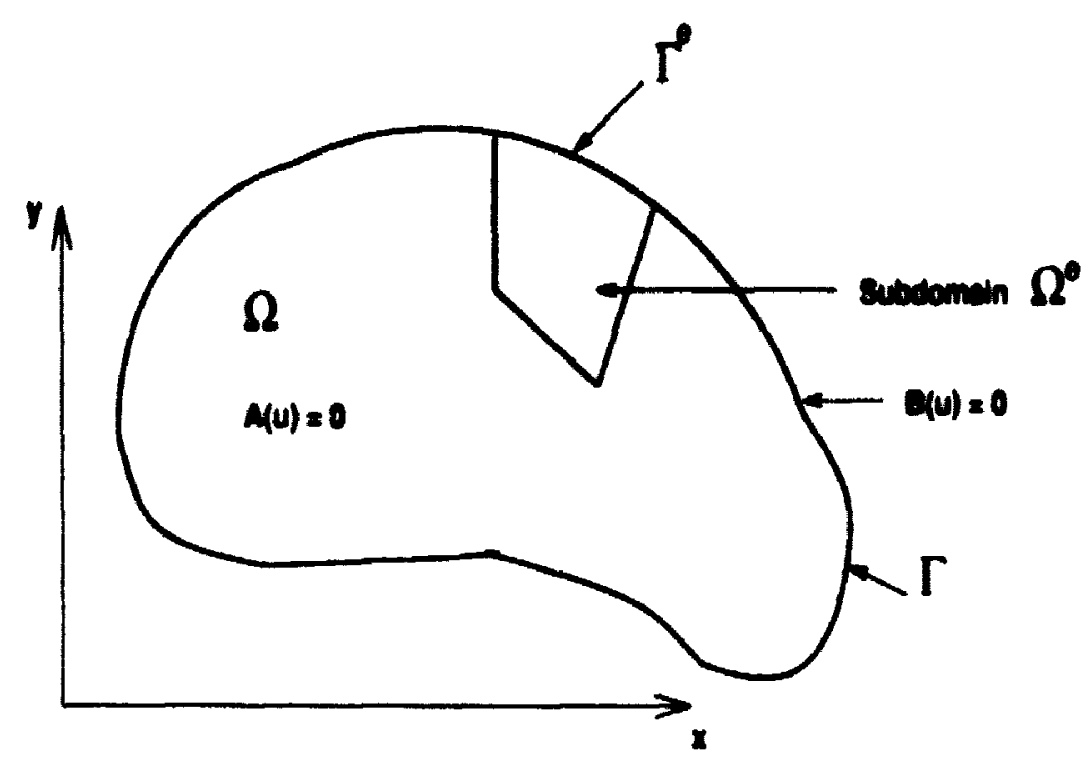

Figure 3.1: Problem domain $\Omega$ and boundary I"

the unknown function sought such that it satisfies a certain differential exuation set in a domain ( volune or area et, , $\Omega$ as shown in Figure 3.1 together with certain boundary conditions on the boundaries of the domain $\mathrm{T}$. The differential and boundary conditions set can then be represented as [39]

$$
\begin{aligned}
& \mathbf{A}(\mathbf{u})=\mathbf{0} \\
& \mathbf{B}(\mathbf{u})=\mathbf{0}
\end{aligned}
$$

The function sougl!t may be a scalar quantity or may represent a vector of several variables. Similarly the differential equation may be a single or a set of simult ane. ous equations. The finite clement process being one of approximation will sork the solution in the approxinate form as 


$$
\mathrm{u} \approx \hat{\mathrm{u}}=\sum_{i}^{n} N_{i} a_{i}=\mathrm{Na} \cdots i=1 \text { to } n
$$

where $\mathbf{N}_{i}$ is the weighting functions prescribed in terms of independent variables (such as co-ordinates $x, y, z)$ and all or some of the parameters $a_{i}$ are unknown. $n$ is the total number of unknowns. Using the weighted residual approach, the integral or weak statement equivalents to the differential equation is

$$
\int v^{*} A(u) d \Omega \equiv \int\left(v_{1} A_{1}(u)+v_{2} A_{2}(u)+\cdots\right) d \Omega=0
$$

where

$$
\mathbf{v}=\left[\begin{array}{c}
v_{1} \\
v_{2} \\
\vdots
\end{array}\right]
$$

is a set of test functions equal in number to the number of equations invalved. It can be stated that if (3.4) is satisfied for any admissible $v$ then differential equation (3.1) must be satisfied at all points of the domain. If the boundary conditions are satisfied simultaneously for any set of functions $v$ then

$$
\int_{\Gamma} v^{e} B(u) d \Gamma \equiv \int_{\Gamma}\left(v_{1} B_{1}(u)+v_{2} B_{2}(u)+\cdots\right) d \Gamma=0
$$

and the integral statement

$$
\int_{\mathbf{n}} v^{\mathbf{l}} A(u) d \Omega+\int_{r} \nabla^{\prime} B(u) d \Gamma=0
$$

is satisfied for all $v, \nabla$ which is equivalent to satisfying differential equations (3.1) and their boundary conditions (3.2). Discussion about choice of $v, \bar{v}, u$ is given in [39]. (3.6) can be rewritten as

$$
\int_{\mathbf{n}} \mathbf{N}, \mathbf{A}(\mathbf{u}) \mathrm{d} \boldsymbol{\Omega}+\int_{\mathbf{r}} \mathbf{N}, \mathbf{E}(\mathbf{u}) \mathrm{d} \boldsymbol{\Omega}=0 \cdots j=1 \text { to } n
$$


where $\mathbf{N}_{j}, \mathbf{N}_{j}$ are suitably defined weighting functions which ensure that as $n \rightarrow \infty$,

$$
\begin{aligned}
& \mathbf{A}(u) \rightarrow 0 \in \Omega \\
& \mathbf{B}(u) \rightarrow 0 \in \Gamma
\end{aligned}
$$

i.e. at all points the approximation tends to the exact solution. A definite integral of the type occurring in (3.7) is simply sum of such integrals occurring on subdomains into which the whole domain is divided. Thus if

$$
\begin{aligned}
& \boldsymbol{\Omega}=\sum_{e=1}^{m} \Omega^{e} \\
& \Gamma=\sum_{e=1}^{m} \Gamma^{e}
\end{aligned}
$$

where $\Omega^{e}$ and $\Gamma^{e}$ are associated with elements into which the problem is divided and $m$ is the total number of elements, then for all finite functions () we have

$$
\begin{aligned}
& \int_{\Omega}() d \Omega \equiv \sum_{e=1}^{m} \int_{r_{e}}() d \Omega \\
& \int_{\Gamma}() d \Gamma \equiv \sum_{e=1}^{m} \int_{\Gamma^{e}}() d \Gamma
\end{aligned}
$$

This allows the whole region to be divided into standard type of subregions where the parameters $a_{1}$ are usually the nodal values of the independent function with the trial functions defined in a local manner. The integrals can then be evaluated element by element and the approximating equations such as (3.7) can be obtained by a simple addition of elcment contributions.

\subsubsection{FEM Applied to Heat Diffusion Problems}

To discuss the application of FEM to the heat diffusion problem (2.8) considering the boundary conditions as

$$
T-\bar{T}=0 \cdots \text { on }_{1}
$$




$$
n^{\prime} k \nabla T-\hat{Q}=0 \cdots o n \Gamma_{2}
$$

where $\Gamma_{1}, \Gamma_{2}$ define parts of the boundary $\Gamma$ on which the temperature $T$ or flux $\dot{Q}$ are given and $\mathbf{n}$ represents the vector of unit magnitude normal to the boundary [40].

If $T_{i}$ is a function defined as one of the independent variables using trial functions defined in terms of the remaining independent variables, which is a semi-discrete process, results in a system of ordinary differential equations.

The semi-discretization process for the three dimensional heat conduction problem (2.8) with boundary conditions (3.11) can be illustrated using the following.

$$
T(x, y, z, \tau) \approx \dot{T}=\sum N_{3}(x, y, z) T_{1}(\tau)
$$

Now weak form of the heat diffusion equation can be explicitly written as,

$$
\int_{\Omega} N_{\jmath}\left\{\nabla^{\imath} \mathbf{k} \nabla \dot{T}+\dot{q}-c \frac{\partial \hat{\imath}}{\partial \tau}\right\} d x d y d z+\int_{\Gamma_{1}} N,(\dot{T}-\bar{T}) d \Gamma-\int_{\Gamma_{2}} N_{\jmath}\left(\mathbf{n}^{2} k \nabla \dot{T}-\hat{Q}\right) d \Gamma=0
$$

Integraling (3.13) by parts and using Green's theorem to obtain an equal order of differentiation results in an ordinary differential equations set

$$
\mathbf{C} \frac{d}{d \tau} \mathbf{r}+\mathbf{G T}+\mathbf{b}=\mathbf{0}
$$

where

$$
\begin{gathered}
\mathbf{G}_{j i}=-\int_{\Omega} \nabla^{\imath} N_{j} \mathbf{k} \nabla N_{i} d x d y d z+\int_{\Gamma} N_{j} \mathbf{n}^{2} \mathbf{k} \nabla N_{i} d \Gamma+\int_{\Gamma_{2}} N_{j} \mathbf{n}^{2} \mathbf{k} \nabla N_{i} d \Gamma \\
\mathrm{C}_{j i}=\int_{\Omega} N_{j} c N_{i} d x d y d z \\
\mathrm{~b}_{j}=\int_{\Omega} N_{j} \hat{q} d x d y d z-\int_{\Gamma_{1}} N_{j} \bar{T} d \Gamma-\int_{\Gamma_{2}} N_{j} \dot{Q} d \Gamma
\end{gathered}
$$

assuming $\hat{Q}$ is due to convective heat exchange (2.9), where $T$, is the same as $T$, conductance matrix $\mathbf{G}$, capacitance matrix $\mathbf{C}$, input vector $\mathbf{b}$ can be written as

$$
\mathbf{G}_{j i}=-\int_{\Omega} \nabla N, k \nabla N_{1} d x d y d z+\int_{r_{2}} N_{j} h N_{i} d \Gamma
$$




$$
\begin{gathered}
\mathbf{C}_{j i}=\int_{\Omega} N_{j} c N_{1} d x d y d z \\
\mathbf{b}_{j}=\int_{\Omega} N_{j} \hat{q} d x d y d z-\int_{\Gamma_{1}} N, \bar{T} d \Gamma-\int_{\Gamma_{2}} N_{j} h T_{f}
\end{gathered}
$$

\subsection{Overview of Numerical Techniques for Time Domain Analysis}

The appearance of nodal temperature as a function of time in (3.14) requires discretization in the time domain as well. Which can be accomplished simply by assuming time increments $\Delta T$ in the computation of (3.14) as shown in Figure 3.2. The expression of the rate of change of temperature however requires an optimum finite difference algorithm to achieve both numerical stability and rapid convergence.

In thermal problems as in structural mechanics, a time varying solution may be obtained by a direct temporal integration or modal method $[41,42,4]$. The modal method is favored if the material properties are not temperature dependent, the solution is dominated by a few of the lowest eigen modes and is needed over a longer time interval. If the problem is nonlinear or the solution displays sharp transicnts (which require many eigen modes for accurate description) and is needed over the shorter time interval, then direct integration is favored.

\subsection{Direct Integration Techniques}

Considering two temperature states separated by a time increment $\Delta \tau$, and denoting them by $T_{n}$ and $T_{n+1}$, at time $r$ and $\tau+\Delta \tau$, a temporal integration schene known as generalized trapezoidal rule can be written based on the assumption that the two temperature states have the relation, 


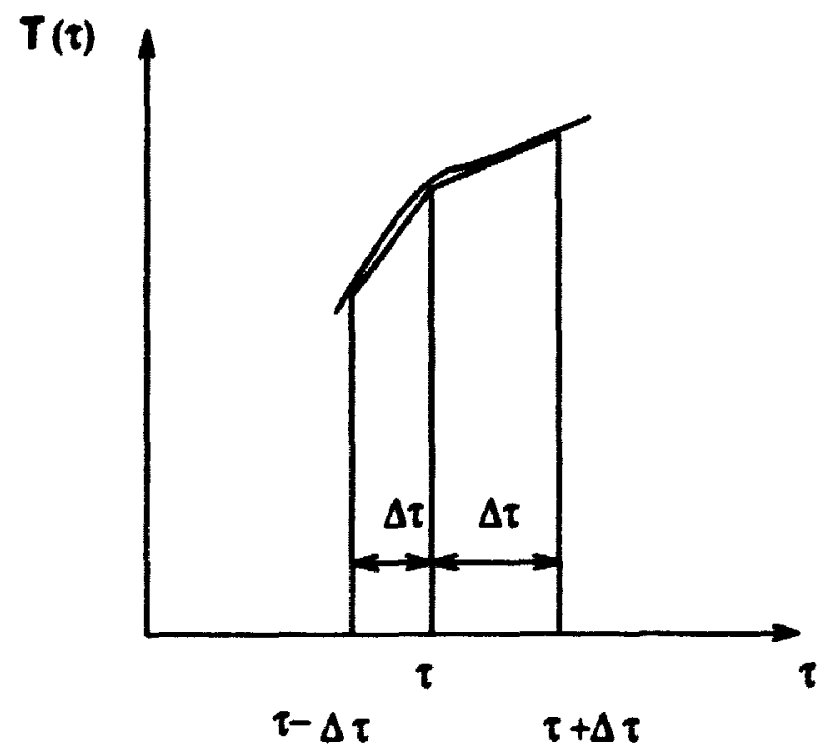

Figure 3.2: Discretization in the time domain

$$
\mathbf{T}_{n+1}=\mathbf{T}_{n}+\left\{(1-\beta) \dot{\mathbf{T}}_{n}+\beta \dot{\mathbf{T}}_{n+1}\right\}(\Delta \tau)
$$

In (3.15) varying the factor $\beta$ results in different integration schemes. Writing (3.14) for time $\tau$ and $\tau+\Delta \tau$ and multiplying the first with $1-\beta$ and the second by $\beta$ results in,

$$
\begin{gathered}
(1-\beta)\left(\mathbf{G} \mathbf{T}_{n}+\mathbf{C} \dot{\mathbf{T}}_{n}\right)=-(1-\beta) \mathbf{b}_{n} \\
\beta\left(\mathbf{G} \mathbf{T}_{n+1}+\mathbf{C} \dot{\mathbf{T}}_{n+1}\right)=-\beta \mathbf{b}_{n+1}
\end{gathered}
$$

adding (3.16) and (3.17) and using (3.15) results in

$$
\left(\frac{\mathbf{C}}{\Delta T}+\beta \mathbf{G}\right) \mathbf{T}_{n+1}=\left(\frac{\mathbf{C}}{\Delta \tau}-(1-\beta) \mathbf{G}\right) \mathbf{T}_{n}-\left\{(1-\beta) \mathbf{b}_{n}+\beta \mathbf{b}_{n+1}\right\}
$$

From a known solution $\mathbf{T}_{o}$ at $\tau=0$, using (3.18) temperature solutions at various time points can be found. For example for 100 different values of $\Delta r$, computational 
effort to obtain the temperature solution is $100 \mathrm{LU}$ decompositions of the coefficient of $\mathbf{T}_{\mathrm{n}+1}$ and 100 forward backward substitutions.

Depending on $\beta$, time step $\Delta \tau$ in (3.18) may have an upper limit if the algorithm is to be numerically stable. If $\beta<0.5$ the largest $\Delta r$ for stability is

$$
\Delta r_{c r}=\frac{2}{(1-2 \beta) \lambda_{\max }}
$$

where $\lambda_{\max }$ is the largest eigenvalue of the system. If $\beta \geq 0.5$ the algorithm is unconditionally stable; that is, stability (but not accuracy) is guaranteed as $\Delta T$ becomes indefinitely large.

$\beta=0$ forward difference or Euler (conditionally stable)

$\beta=0.5$ Crank-Nicolson or trapezoidal rule (unconditionally stable)

$\beta=\frac{2}{3}$ Galerkian (unconditionally stable)

$\beta=1$ backward difference (unconditionally stable)

Explicit Methods: If $\beta=0$, the algorithm is termed explicit method. Explicit integration methods are used to generate the solution at the next time point directly. They are mainly to predict the starting values for other formular like backward Euler or trapezoidal rule.

Implicit Methods: If $\beta>0$ in (3.18) the integration scheme is termed implicit method. If $\mathbf{C}$ is a diagonal matrix and $\beta=0$, the computational effort per time step is small but so is $\tau_{\text {cr. }}$. The choice $\beta=0.5$ is popular but, sharp transients may causeoscillations in the solution. Oscillations can be reduced by using a smaller value of $\Delta \tau$ or can be numerically damped by using a value of $\beta$ slightly gitat.. than 0.5 . 


\subsection{Modal Method}

Considering the eigen problem for (3.14)

$$
(\mathbf{G}-\lambda \mathbf{C})\{\mathbf{T}\}=0
$$

and if each eigen vector $\mathbf{T}_{t}$ is normalized with respect to $\mathbf{C}$, that is, if $\mathbf{T}_{1}^{t} \mathbf{C} \overline{\mathbf{T}}_{i}=\mathbf{1}$, then

$$
\begin{aligned}
\Phi^{\prime} \mathbf{C} \Phi & =\mathbf{I} \\
\Phi^{\prime} \mathrm{G} \Phi & =\lambda
\end{aligned}
$$

where $\Phi$ is the modal matrix: that is a matrix whose columns are the normalized eigenvectors $\bar{T}_{1}, \overline{\mathbf{T}}_{2}$, etc... I is a unit matrix, and $\lambda$ is the diagonal spectral matrix. Nodal temperatures are transformed to generalized temperatures $\mathbf{Z}$ by

$$
\mathbf{T}=\boldsymbol{\Phi} \mathbf{Z}
$$

Substituting (3.22) into (3.14), premultiplied by $\Phi^{t}$, gives

$$
\Phi^{t} C \frac{d}{d \tau} \boldsymbol{\Phi} Z+\Phi^{t} G \Phi Z+\Phi^{t} b=0
$$

further substitution of (3.21) results in uncoupled equations, each having the form

$$
\dot{Z}_{1}+\lambda_{1} Z_{i}=p_{i}
$$

where $p_{i}=-\Phi_{1}^{t} b_{1}$. $\Phi_{1}$ is the $i^{\text {th }}$ column of $\Phi$, and $i$ is 1 to $m$, where $m$ is typirally much less than the total number of degrees of freedom; that is, only the first few columns of $\Phi$ are used. By integrating (3.24) $Z_{1}=C_{1} \epsilon^{-\lambda_{1} r}+\frac{p_{1}}{\lambda_{1}}$ is known, and using (3.22) $T$, can be calculated. 


\subsection{Summary}

Finite element method basics have been reviewed. The steps involved in obtaining a linear set of algebraic equations for solving heat diffusion problems, in the time domain have been discussed. An overview of the conventional numerical techniques for solving these equations has been presented. 


\section{Chapter 4}

\section{Moment Matching Technique}

\subsection{Introduction}

Moment matching technique was introduced in linear systems analysis as a form of model reduction. This method extracts a small approximate set of dominant poles and residues for a large electrical network that may contain hundreds of actual poles. For example if a system has poles at -1 and -1000 the dominant pole would be the pole at -1 . Moment matching method approximates the transient response of a network by first expanding the system equations in moments (coefficients) of a Taylor series expansion about $s_{:}=0$ in the frequency domain, and then matching the first $2 q$ moments of the series to a low order $q$-pole model. The cost of an expansion is approximately one frequency point analysis. In this chapter concepts of the momentmatching technique are presented.

\subsection{Moment Matching Method}

Moment methods consists of two main steps: a) moment generation and b) moment matching. Moment generation is the process by which a set of linear network 
equations is expanded in a Taylor series. The coefficients of the expansion are known as moments because they relate to the time moments of the transfer function. One of the approaches in generating the system moments is to use a recursive relationship. requiring only one decomposition of the system equations. This is approximately equal in CPU time for one DC solution of the network. The extra benefit of doing the analysis is, the moments for every output variable in the original equations are calculated simultaneously. The moments for a selected output can be extracted simply, while information at ot her outputs is readily available.

Moment matching is a technique where a set of coefficients of a low-order approximate rational transfer function are found such that the moments of the approximate function matches a given number of initial moments of the original function. This is achieved through a set of linear equations that determine the coefficients of the denominator polynomial. Given those coefficients there is an option of either determining the coefficients of the numerator polynomial of factoring the denominator and calculating a set of residues to match the resultant $q$ poles. Together, moment generation and moment matching are generally referred to as the moment matching techniques.

\subsubsection{System Moments}

Taking the Laplace transform of (3.14),

$$
(\mathbf{G}+\mathbf{C s}) \hat{\mathbf{T}}(s)=\mathbf{b}
$$

where $\hat{\mathbf{T}}(s)$ is the Laplace transform of $\mathbf{T}(+)$. The impulse response. $\hat{\mathbf{T}}(s)$ can be. expanded in Taylor series about $s=0$, in the form [11] 


$$
\hat{\mathbf{T}}(s)=\sum_{n=0}^{\infty} \mathbf{M}_{n} s^{n}
$$

where $M_{n}$ is a vector representing the $n t h$ moment of $\hat{\mathbf{T}}(s)$. For a linear lumped network, the moments can be evaluated recursively, as follows

$$
\begin{gathered}
\mathbf{G M}_{0}=\mathbf{b} \\
\mathbf{G M}_{n}=-\mathbf{C M}_{n-1}
\end{gathered}
$$

The transfer function $\hat{T}_{1}(s)$ of a selected output $i$ is given by

$$
\hat{T}_{i}(s)=m_{0 t}+s m_{11}+s^{2} m_{2 i}+\cdots
$$

where $m_{n t}=\left[\mathbf{M}_{n}\right]_{1}$. The moments $m_{n i}$ are identical to the time domain moments of the impulse response of a particular output, $T_{i}(\tau)=\mathcal{L}^{-1}\left(\hat{T}_{i}(s)\right)$. In subsequent sections subscript $i$ will be dropped for simplicity.

\subsubsection{Generating an Approximate Response}

The form of an actual one input, one output transfer function of an output can be described as,

$$
\dot{T}(s)=\frac{P(s)}{Q(s)}
$$

where $P(s)$ and $Q(s)$ are polynomials in $s$ in which the degree of the numerator is less than or equal to the degree in the denominator. Assuming the degree of the numerat or is one less than the degree of the denominator, partial fraction decomposition on $(4.6)$ yields. 


$$
\hat{T}(s)=\sum_{j=1}^{N_{p}} \frac{k_{j}}{s-p_{j}}
$$

$N_{p}$ is the number of actual poles $p_{j}$, of the system and the number of residues, $k_{j}$, of the selected output. While the poles of the system are same at every output node. the residues may vary from node to node. $\dot{T}(s)$ may also be decomposed into $N_{p}$ poles and $N_{z}$ zeros,

$$
\hat{T}(s)=\frac{\left(s-z_{1}\right)\left(s-z_{2}\right) \cdots\left(s-Z_{N_{2}}\right)}{\left(s-p_{1}\right)\left(s-p_{2}\right) \cdots\left(s-p_{N_{2}}\right)}
$$

The time domain impulse response of the output can be given in closed form in terms of the poles and residues as,

$$
T(\tau)=\sum_{j=1}^{N_{\nu}} k_{j} \epsilon^{p, \tau}
$$

For a system with large number of poles, $N_{p}$, and therefore the order of $Q(*)$, can be of the order of hundreds and thousands. Generating $\Lambda_{r}$ poles of even considerably small system is CPU intensive.

Using the moments generated, a low-order q-pole approximation. which is a from of Padé approximation $[11.12,13]$ to $(4.7)$ can be constructed in the form,

$$
\hat{T}(s) \approx \frac{\dot{P}(s)}{\hat{Q}(s)}=\frac{\left(s-\dot{\xi}_{1}\right)\left(s-\dot{\xi}_{2}\right) \cdots\left(s-\dot{\xi}_{q}\right)}{\left(s-\dot{p}_{1}\right)\left(s-\dot{p}_{2}\right) \cdots\left(s-\dot{p}_{q}\right)} \quad q \ll N_{p}
$$

where $\dot{z}$, and $\dot{p}$, are the approximate number of zeros and poles. Fipuation (4.11) can be rewritten in the form.

$$
\hat{T}(s) \approx \sum_{n=0}^{24-1} m_{n} s^{n} \approx \sum_{j=1}^{q} \frac{\hat{k}_{j}}{s-\dot{p}_{j}}
$$

In the time domain. an approximate impulse response can be then given by, 


$$
T(\tau)=\sum_{j=1}^{q} \dot{k}_{j} \epsilon^{\dot{p}_{j} \tau}
$$

Similar closed forms are also possible for an approximate step response, ramp response, or exponential response, by multiplying the input function by the impulse response in the frequency domain and decomposing the result into partial fractions. All other complex input waveforms may be decomposed into a linear combination of these simpler forms.

\subsubsection{Poles and Residues}

In order to obtain $\hat{p}_{3}$ and $\hat{k}$, considering the characteristic polynomial of the reduced order model as.

$$
\sum_{i=0}^{q} a_{1} p_{1}^{q-1}=0
$$

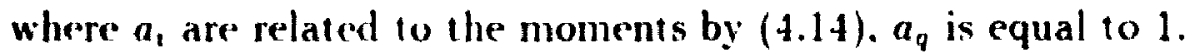

$$
\left[\begin{array}{ccccc}
m_{0} & m_{1} & m_{2} & \cdots & m_{q-1} \\
m_{1} & m_{2} & m_{3} & \cdots & m_{q} \\
\vdots & \vdots & \vdots & \cdots & \vdots \\
m_{q-1} & m_{q} & m_{q+1} & \cdots & m_{2 q-2}
\end{array}\right]\left[\begin{array}{c}
a_{0} \\
a_{1} \\
\vdots \\
a_{q-1}
\end{array}\right]=-\left[\begin{array}{c}
m_{q} \\
m_{q+1} \\
\vdots \\
m_{2 q-1}
\end{array}\right]
$$

The roots of the characteristic polynomial (poles) can be found by using a standard root finding algorithm. The corresponding residues are obtained from

$$
\left[\begin{array}{ccccc}
p_{1}^{-1} & p_{2}^{-1} & p_{3}^{-1} & \cdots & p_{q}^{-1} \\
p_{1}^{-2} & p_{2}^{-2} & p_{3}^{-2} & \cdots & p_{q}^{-2} \\
\vdots & \vdots & \vdots & \cdots & \vdots \\
p_{1}^{-q} & p_{2}^{-q} & p_{3}^{-q} & \cdots & p_{q}^{-q}
\end{array}\right]\left[\begin{array}{c}
k_{1} \\
k_{2} \\
\vdots \\
k_{q}
\end{array}\right]=-\left[\begin{array}{c}
m_{0} \\
m_{1} \\
\vdots \\
m_{q-1}
\end{array}\right]
$$

The time domain impulse response of the approximating model could be evaluated directly as given by $(4.12)$. 


\subsection{Computational Effort for Moment Matching Method}

The overall computational effort for the method is one $L I$ decomposition of the $G$ matrix and $2 q$ forward and backward substitutions for (4.3) and (4.4). Computational effort involved in solving (4.14) and (4.15) is negligible. as the dimensions of the matrices in (4.14) and (4.15) are small as compared to the overall network matrix $\mathbf{G}$. 


\section{Chapter 5}

\section{Thermal Modeling Based on FEM and Moment Matching Method}

\subsection{Introduction}

The finite element method is one of the widely used techniques for thermal analysis of electronic systems, specially due to its ability in modeling complex geometries. Space/time domain formulation of the heat transfer problem using FEM, results in a set of ordinary differential equations, which are solved in the time domain. Usage of conventional solution algorithms is restricted by computing time, since it is often necessary to take very small time step size to avoid numerically induced oscillations. To improve the computational efficiency an effort is made to apply the moment matching method for the solution of thermal finite element equations. This chapter describes the procedure involved in generating a netlist of resistors, capacitors etc., from the finite element matrices generated using a commercial software $[14,15,16]$. The netlist will be given as an input to the electrical circuit simulator implementing the moment matching technique. Transient temperature results for a few examples are presented to illust rate the method. 
In order to avoid the duplication of effort in generating the thermal finite element matrices a commercial software [14] has been chosen with the following features. NISA [14] (Numerically Integrated Elements for System Analysis) is a general purpose finite element program to analyze a wide spectrum of problems such as heat transfer, dynamics, fluid flow problem etc. Finite Element Analysis of Printed Circuit Boards and Packages (FEAP) [15] is a program for the analysis of printed circuit boards and electronic systems. FEAP is interfaced with NISA for thermal analysis with the features which include generation of finite element models of the PCBs interactively using a large built-in library of commercial components such as: dualin-line packages (DIPs), hybrid packages, 'at packs, leadless ceramic chip carriers, etc. Once all components are defined, a finite element mesh is automatically generated for each chip and the entire board. Automatic mesh refinement for an area of interest can be specified. Appropriate elements (e.g. convection link element, radiation element, conduction element) for heat transfer analysis are chosen from the NISA element library.

NISA and FEAP were chosen for generating finite element matrices, due to the above mentioned capabilities. The software stores the finite element matrices in a binary format for further processing. A special program BINTOASCII [16] was given by the company for converting the matrices written in a binary format to ASCII format. The matrices stored in ASCII format are further processed and converted to a electrical netlist format using NETGEN [43] to be given as an input to the electrical simulator. Several test cases have been executed to cherk the arcurary of the extracted finite element matrices. Further, test cases have bern executed to check the accuracy of the electrical netlist. The factors considered in developing FE models for the test cases are: type of elements, such as three dimensional, two 
dimensional, convection elements etc., mesh size and the type of boundary conditions. The extraction procedures were found to be accurate.

\subsection{Generating the Electrical Netlist From the Fi- nite Element Matrices}

Finite element formulation of heat conduction equation (2.8) results in a set of ordinary differential equations. Where $\mathbf{C}$ is the thermal capacitance matrix, $\mathbf{G}$ is the thermal conductance matrix and the $b$ is the input vector. Laplace transformation of (2.8) and calculation of the resulting system moments was discussed in Chapter 4. To obtain the system moments the finite element nuatrices in (2.8) will be transformed to an electrical net list consisting of resistors, capacitors and voltage controlled current sources. Considering the thermal capacitance matrix $\mathbf{C}$ of dimension $\boldsymbol{n}$ each diagonal entry (ith row $j$ th column, where $i=j$ ) will be represented as a capacitor between node $i$ and node $n+(2 i-1)$. To measure the current in the capacitor a resistor of small value will be added between node $n+(2 i-1)$ and $n+2 i$. A negative resistor of the same value will be added between node $n+2 i$ and ground (denoted as node 0 ). Each non zero off-diagonal entry in the row $i$ will be represented as a voltage controlled current source. A diagonal element from the thermal conductance matrix $\mathbf{G}$ will be represented as a conductor between node $i$ and node $j$. Each off diagonal entry will be represented as a voltage controlled current source. The process of extracting netlist will be explained using an example in the following section.

\subsubsection{Example}

Figure 5.1 shows an axisymmetric heat conduction problem. Finite element discretization for the same is shown in Figure 5.2. Based on the finite element formula- 


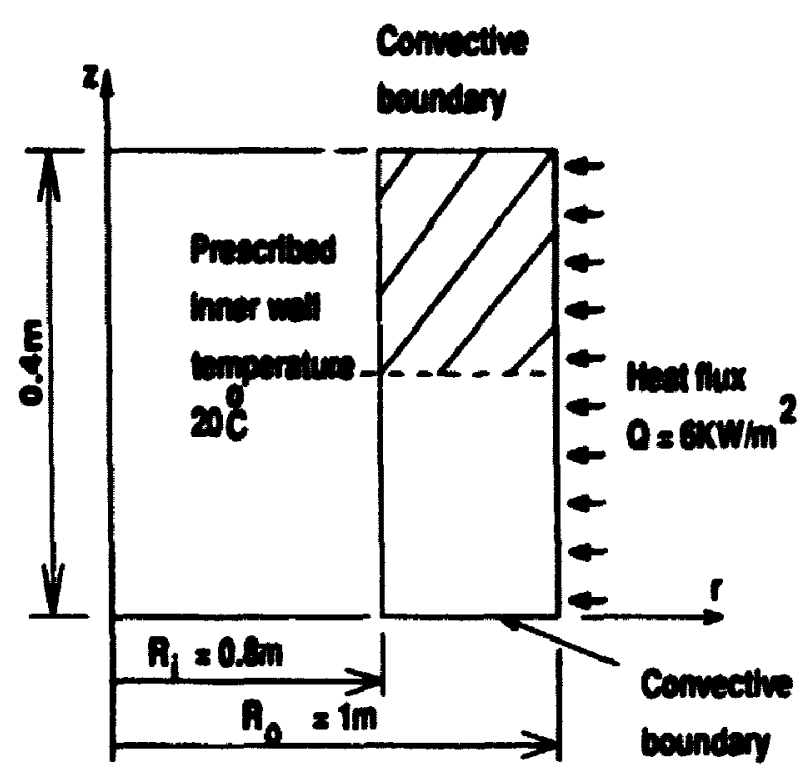

Figure 5.1: Dimensions and boundary conditions

tion presented in Chapter 3 , the matrices $\mathbf{C}, \mathbf{G}$ and $\mathbf{b}$ were calculated and are given as follows.

$$
\begin{aligned}
& \mathbf{C}=\left[\begin{array}{ccccc}
0.5979 e+0.5 & 0.1601 \epsilon+05 & 0 & 0.1459 \epsilon+05 & 0.3061 \epsilon+05 \\
& 0.6834 \epsilon+0.5 & 0.1744 \epsilon+05 & 0 & 0.3345+0.5 \\
S Y M & & 0.6834 \epsilon+05 & 0.1601 \epsilon+05 & 0.3345+05 \\
& & & 0.5979 \epsilon+0.5 & 0.3061 \epsilon+05 \\
& & & & 0.1281 \epsilon+05
\end{array}\right] \\
& \mathbf{G}=\left[\begin{array}{ccccc}
0.3485 \epsilon+03 & 0 & 0 & 0 & -0.3485++03 \\
& 0.3753 \epsilon+03 & 0 & 0 & -0.3753 \epsilon+03 \\
S Y M & & 0.3777 \epsilon+03 & 0.1130 \epsilon+01 & -0.3753 \epsilon+0.3 \\
& & & 0.3506 \epsilon+03 & -0.3485 \epsilon+03 \\
& & & & 0.1447 \epsilon+04
\end{array}\right]
\end{aligned}
$$




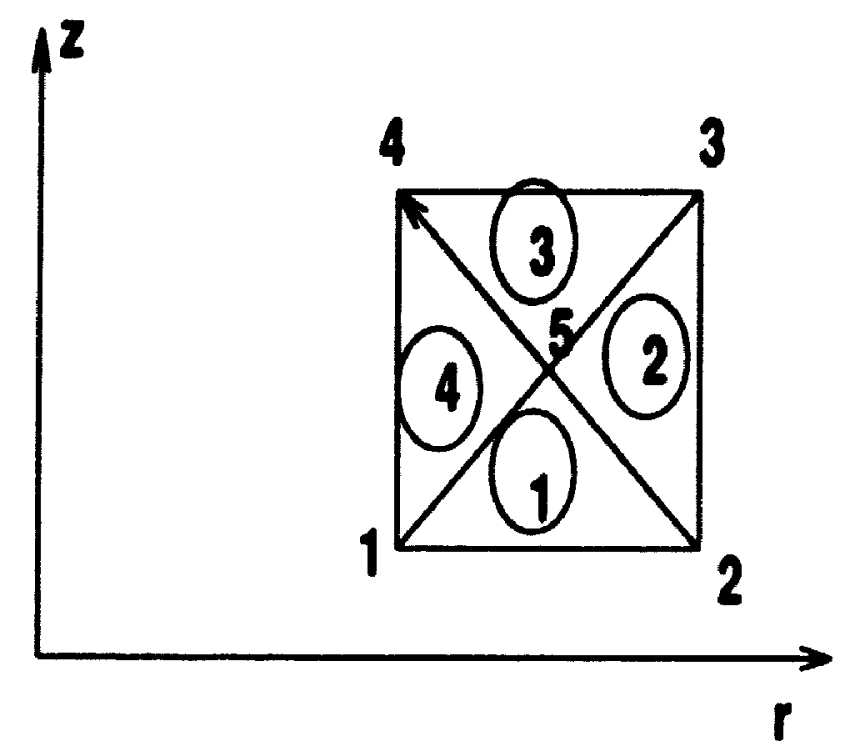

Figure 5.2: Axisymmetric finite element model

$$
\mathbf{b}=\left[\begin{array}{c}
0 \\
3.769 \epsilon+03 \\
3.699 \epsilon+03 \\
0 \\
-67.83
\end{array}\right]
$$

Figure 5.3 shows equivalent electrical circuit representation for the row $i=1$ of the matrix $\mathbf{C}$.

\subsection{Summary of the Netlist Extraction Procedure}

This section summarizes the steps involved in generating an electrical netlist from the finite element matrices.

Step1 Create finitc element model of a PCB using FEAP

Step2 Perform transient thermal analysis using NISA. This step creates the C. $\mathbf{G}$ and $\mathbf{b}$ matrices in binary format. 


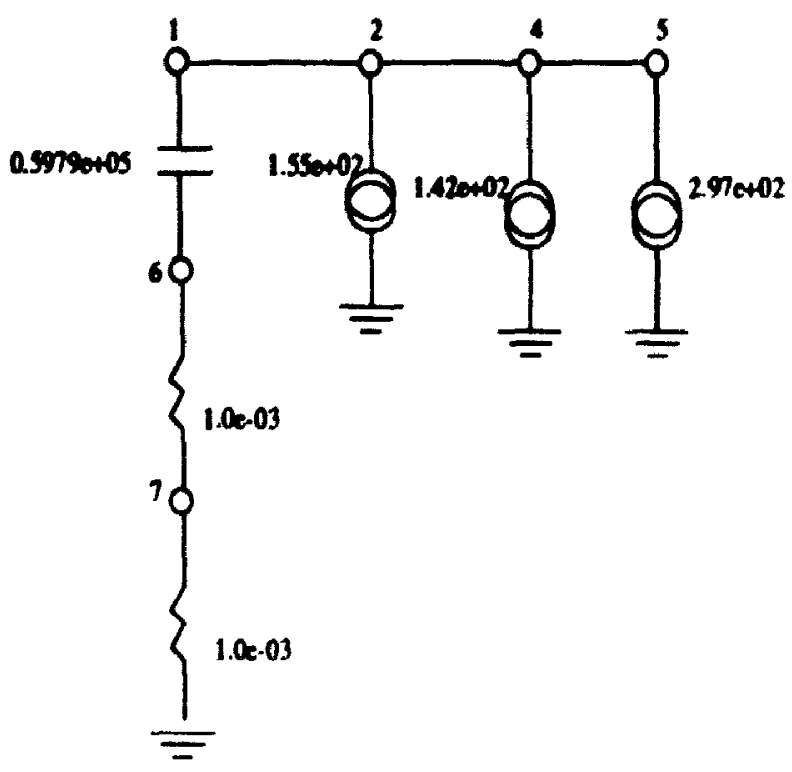

Figure 5.3: Equivalent electrical circuit representation for $i^{\text {th }}$ row of thermal capaci. tance matrix $\mathbf{C}$

Step3 Use BINTOASCII to convert matrices written in binary to ASC 'Il format.

Step4 Generate electrical netlist using NETGEN by giving ASC'II formatled ma. trices as input.

Step5 Electrical netlist created from the above step will be given as the input to the circuit simulator program which has the option of using moment-matrhing method for solution.

\subsection{Examples of Printed Circuit Boards and Pack- ages}

Comparison between the results obtained using the finite clement methorl and the experiments established the reliability and accuracy of the FEM results (c.g. see [44]). In this section. few examples are considered to study the appliation of 
moment-matching method for solving time domain thermal finite element (FE) equations. The solution procedure is based on the discussion presented in the previous sections. A comparison of performance for the moment-matching technique and currently employed integration techniques is made and the results discussed.

Example 1 is a three dimensional model of a PCB with three dual-in-line packages (DIPs). Examples 2 and 3 are two dimensional models of a component on board problems. The transient temperature results obtained using the proposed method are compared with the results from NISA finite element software [14].

\subsubsection{Example1: Printed Circuit Board with 3 Dual-in-Line Packages}

A three layered composite PCB $(0.127 m \times 0.1016 m)$ with three 18 pin dual-inline packages (DIP) mounted on its top face [14] each with a $1 W$ source is shown in Figure 5.4. The board section is a epoxy-glass coupon with $7.62 \times 10^{-4} \mathrm{~m}$ thick layer of copper. The package dimensions are $2.032 \times 10^{-2} \mathrm{~m}$ length and $0.762 \times 10^{-2} \mathrm{~m}$ width respectively. Nine pins $\left(k=21.26 \mathrm{~W} / \mathrm{m}^{\circ} \mathrm{C}\right)$, each across the length conduct heat to the board. Each pin is $0.127 \times 10^{-2} \mathrm{~m}$ wide, $0.0254 \times 10^{-2} \mathrm{~m}$ thick and $0.3048 \times 10^{-2} \mathrm{~m}$ long. The finite element model is shown in Figure 5.5 which consists of 3625 nodes and 3561 elements. The boundary conditions include, convection from the top faces of three DIPs and a fixed temperature of $20^{\circ} \mathrm{C}$ at edges 1 and 3 .

Transient temperature profiles are obtained assuming an initial temperature of $20^{\circ} \mathrm{C}$ at all nodes, using implicit integration [14] and moment-matching techniques to solve the ordinary differential equations resulting from finite element formulation. Using moment-matching technique, it was sufficient to extract 4 poles to represent the system. A comparison of transient temperature response at a few selected nodes 

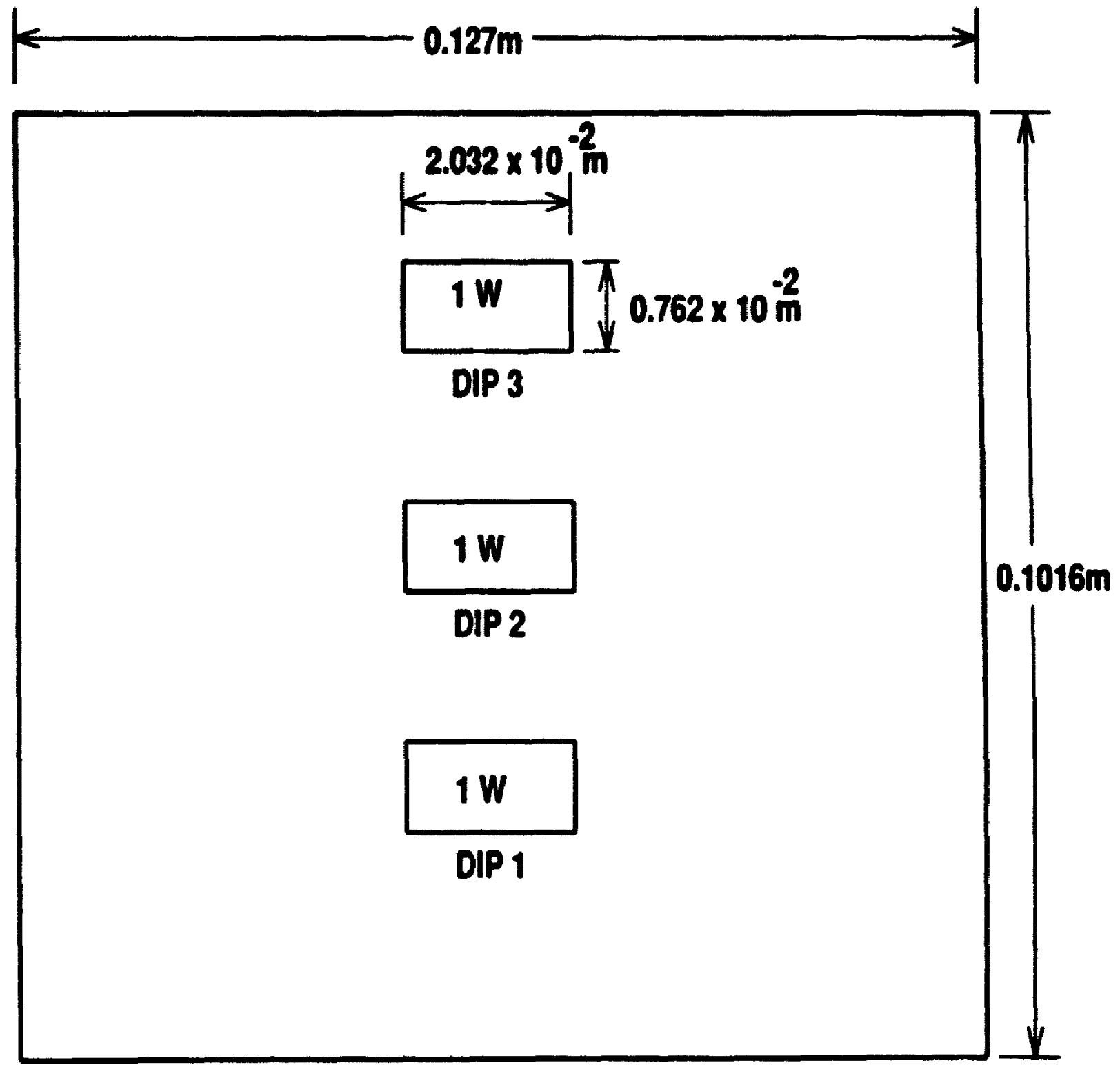

Figure 5.4: Dual-in-line packages on a printed circuit board (Example I) 


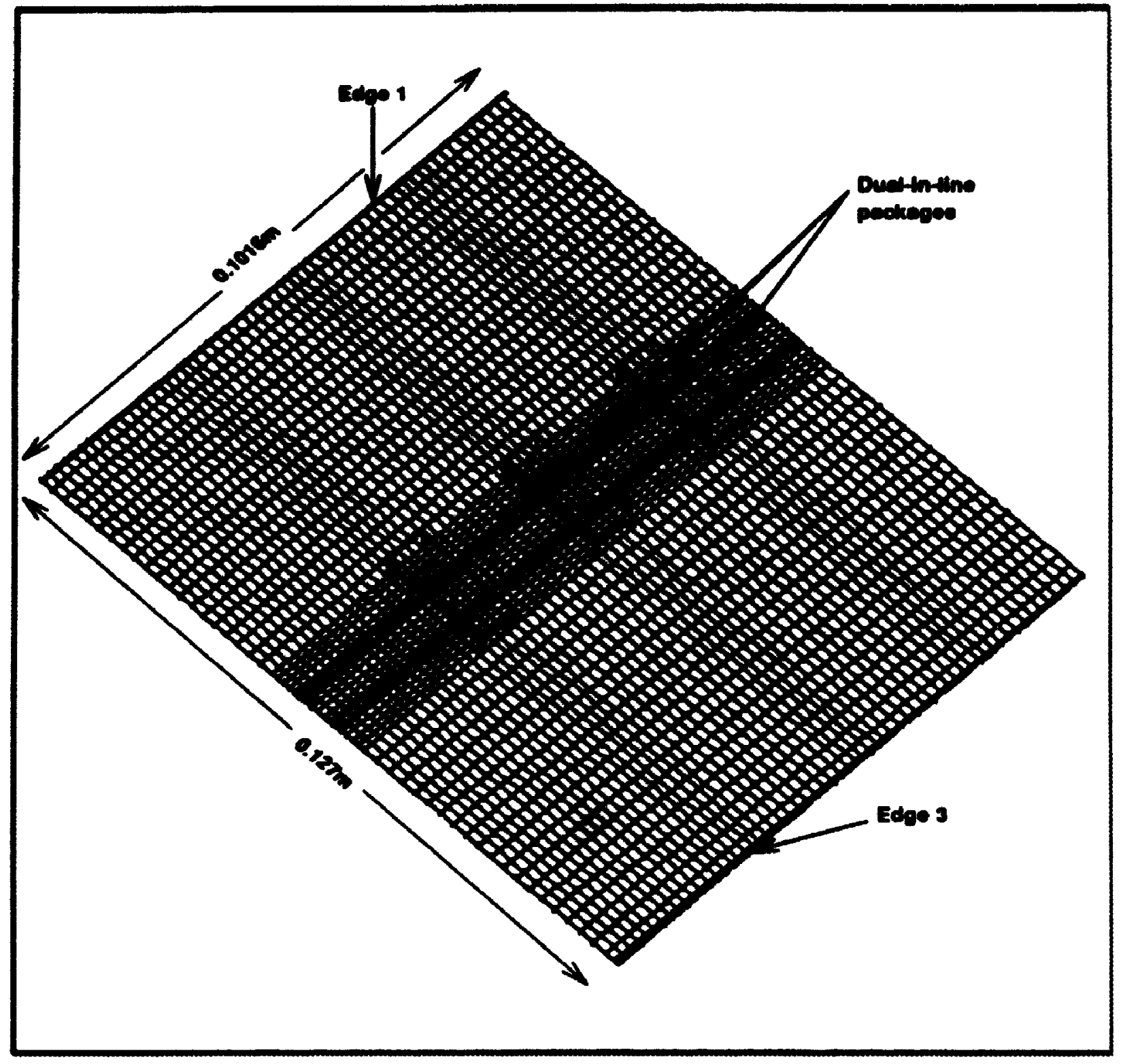

Figure 5.5: Finite element discretization of the PCB in Example 1 


\begin{tabular}{lcccccc}
\hline Method & CPU time & \multicolumn{5}{c}{ nodal temperature in $\left({ }^{\circ} \mathrm{C}\right)$} \\
\cline { 2 - 6 } & (seconds) & $T_{29}$ & $T_{32}$ & $T_{40}$ & $T_{25 s}$ & $T_{400}$ \\
\hline $\begin{array}{l}\text { implicit integration } \\
\text { technique }\end{array}$ & 6803.89 & 45.56 & 45.63 & 47.19 & 33.22 & 36.51 \\
$\begin{array}{l}\text { moment-matching } \\
\text { technique }\end{array}$ & 50.15 & 45.81 & 45.63 & 47.19 & 33.22 & 36.51 \\
\hline
\end{tabular}

Table 5.1: Computational statistics and nodal temperature solution at $\tau=1500$ (seconds) for the printed circuit board example in Fig. 5.4

on the DIPs and on the board is shown in Figures 5.6 and 5.7, which shows the results are in good agreement. Computational statistics for implicit integration and moment-matching techniques are given in Table 5.1. Using moment-matching technique resulted in a speed-up factor of 136 .

\subsubsection{Example2: Single Component on Board}

A problem of a single hypothetical surface mount component on a board section [19] is shown in Fig. 5.8. The board section is a $0.0762 m \times 0.0762 m$ epoxy-glass coupon $\left(k=0.276 \mathrm{~W} / \mathrm{m}^{\circ} \mathrm{C}\right)$ with a $3.56 \times 10^{-5} \mathrm{~m}$ thick layer of solid copper $(k=$ $393.7 \mathrm{~W} / \mathrm{m}^{\circ} \mathrm{C}$ ). The package is represented by a $2.54 \times 10^{-2} \mathrm{~m}$ by $2.54 \times 10^{-2} \mathrm{~m}$ blork. Twenty copper leads per edge conduct heat to the board. Fach lead is $7.62 \times 10^{-2} \mathrm{~m}$ wide, $2.54 \times 10^{-4} \mathrm{~m}$ thick, and $5.08 \times 10^{-4} \mathrm{~m}$ long (from component to board). $A$ single $2.54 \times 10^{-3} \mathrm{~m} \times 2.54 \times 10^{-3} \mathrm{~m}, 1 \mathrm{~W}$ source is centered at the lower surface of the component (nearest to the board). A $5.08 \times 10^{-4} \mathrm{~m}$ air gap separates the component and board.

Axisymmetric finite element model for the above example is shown in Figure 5.9. The chip source, package and board test section radii were calculated on the basis 


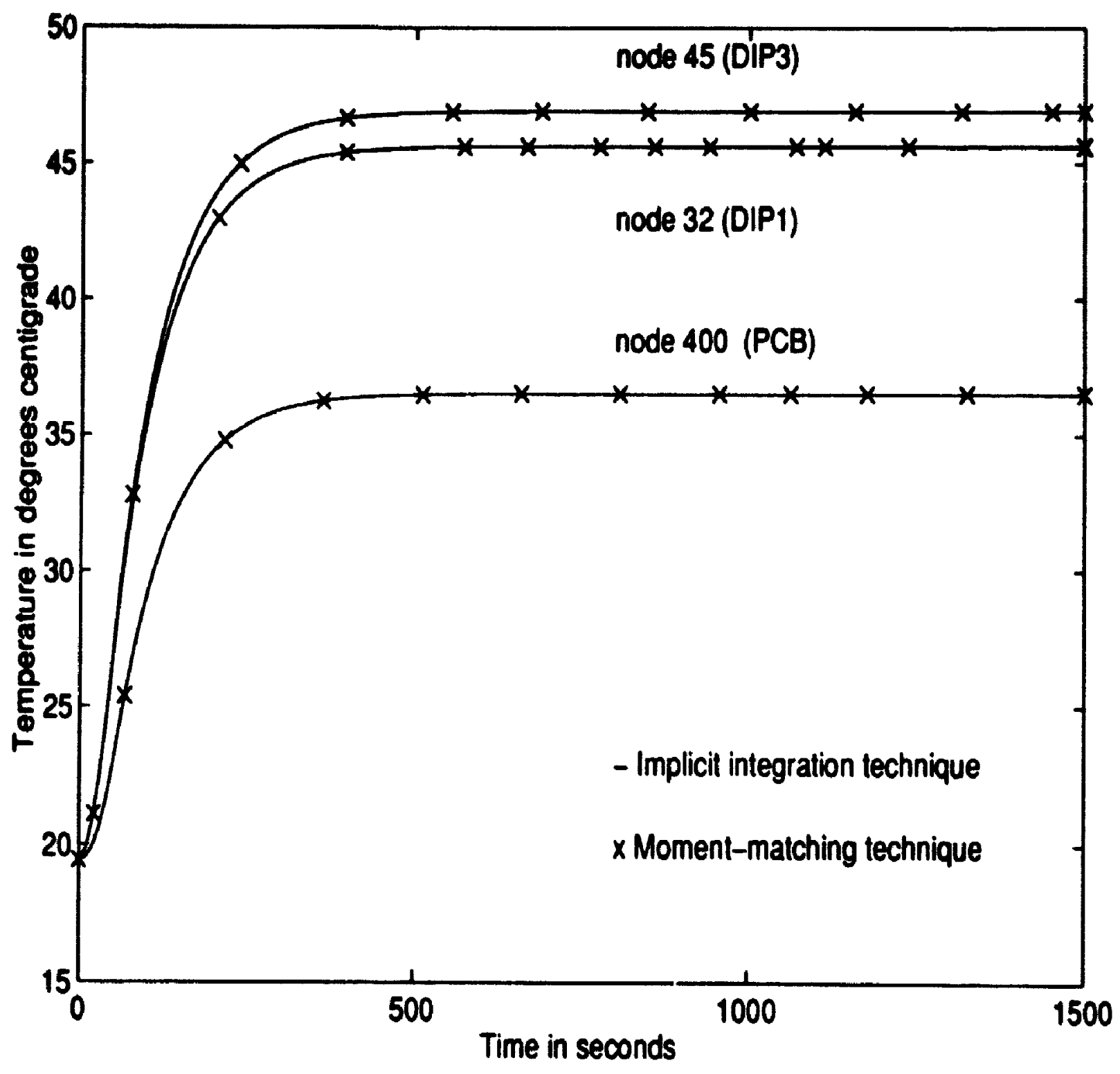

Figure 5.6: Transient temperature response (Example 1) 


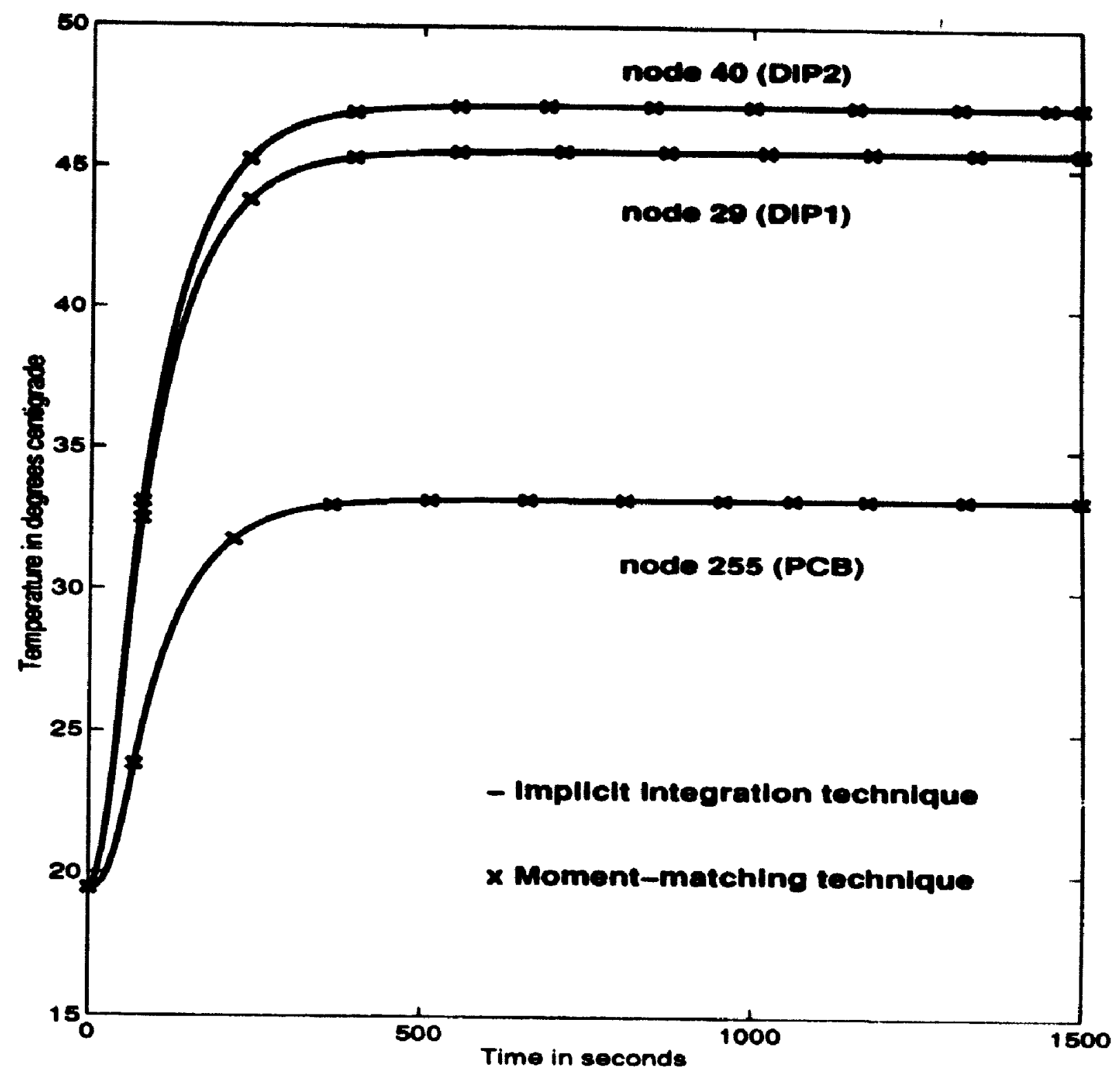

Figure 5.7: Comparison of temperature response using moment-matching and implicit integration techniques (Example 1) 

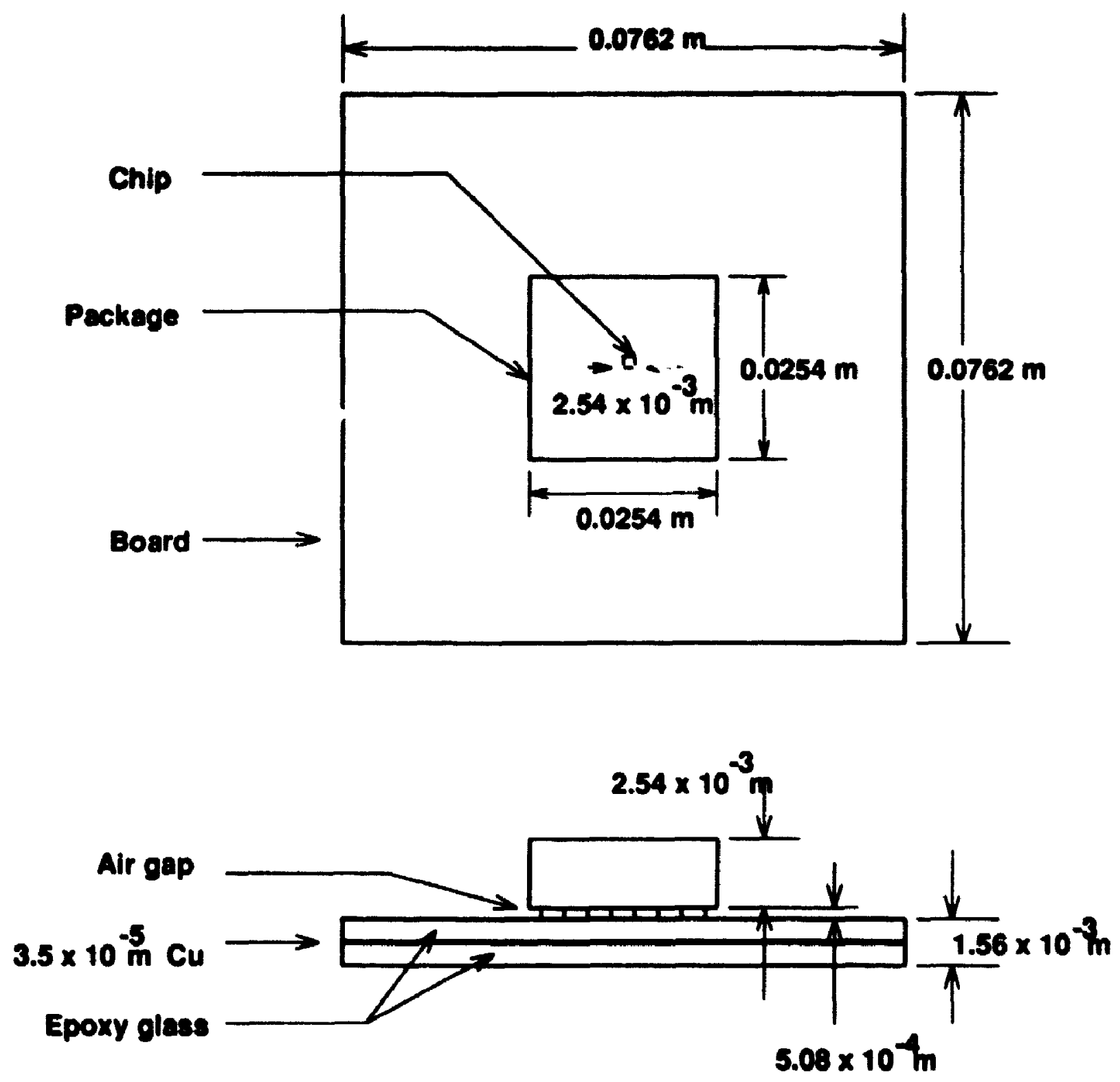

Figure 5.8: A printed circuit board with a surface mount component (Example 2) 


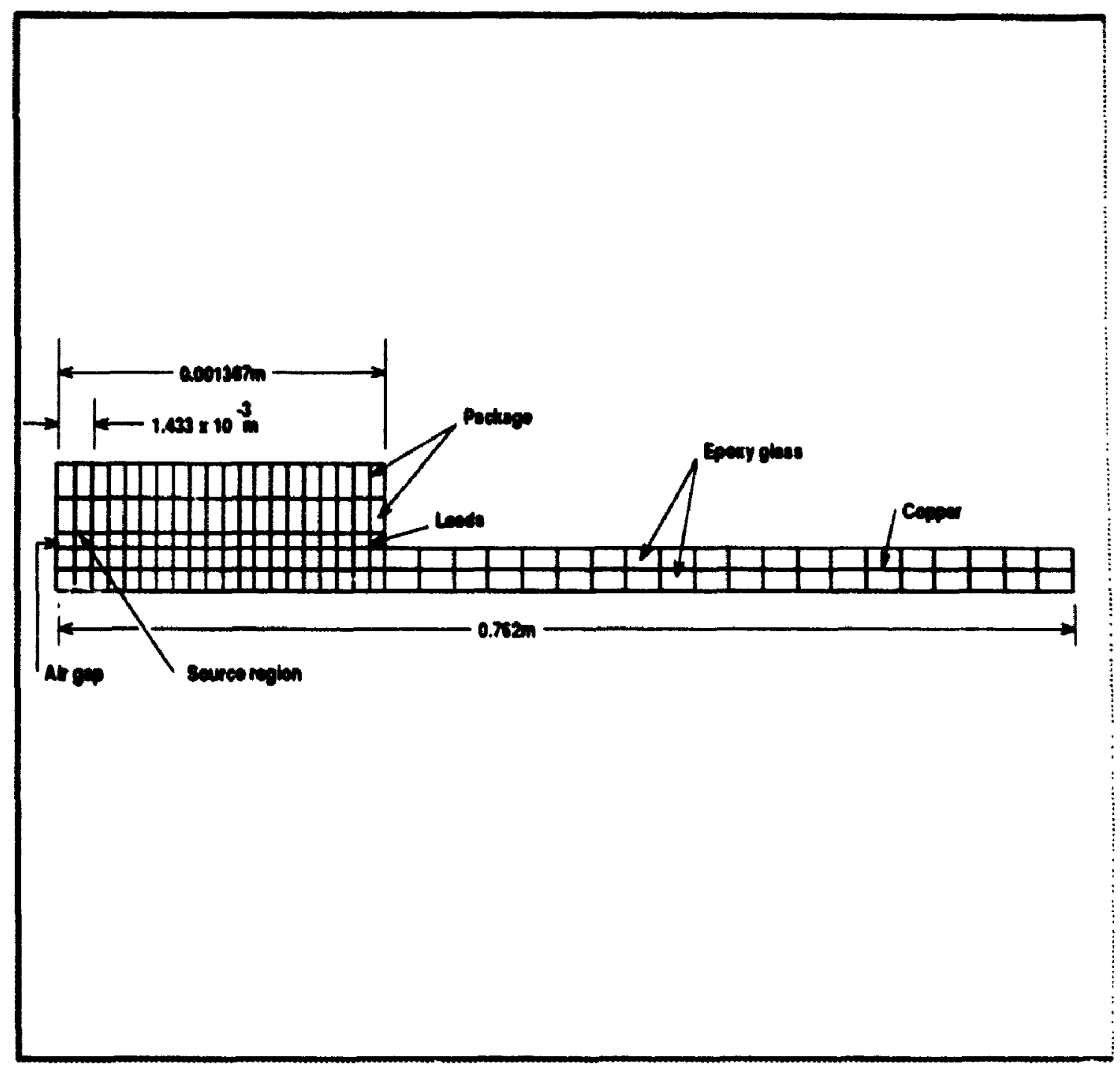

Figure 5.9: Axisymmetric finite element model of a PC 13 in example 2 


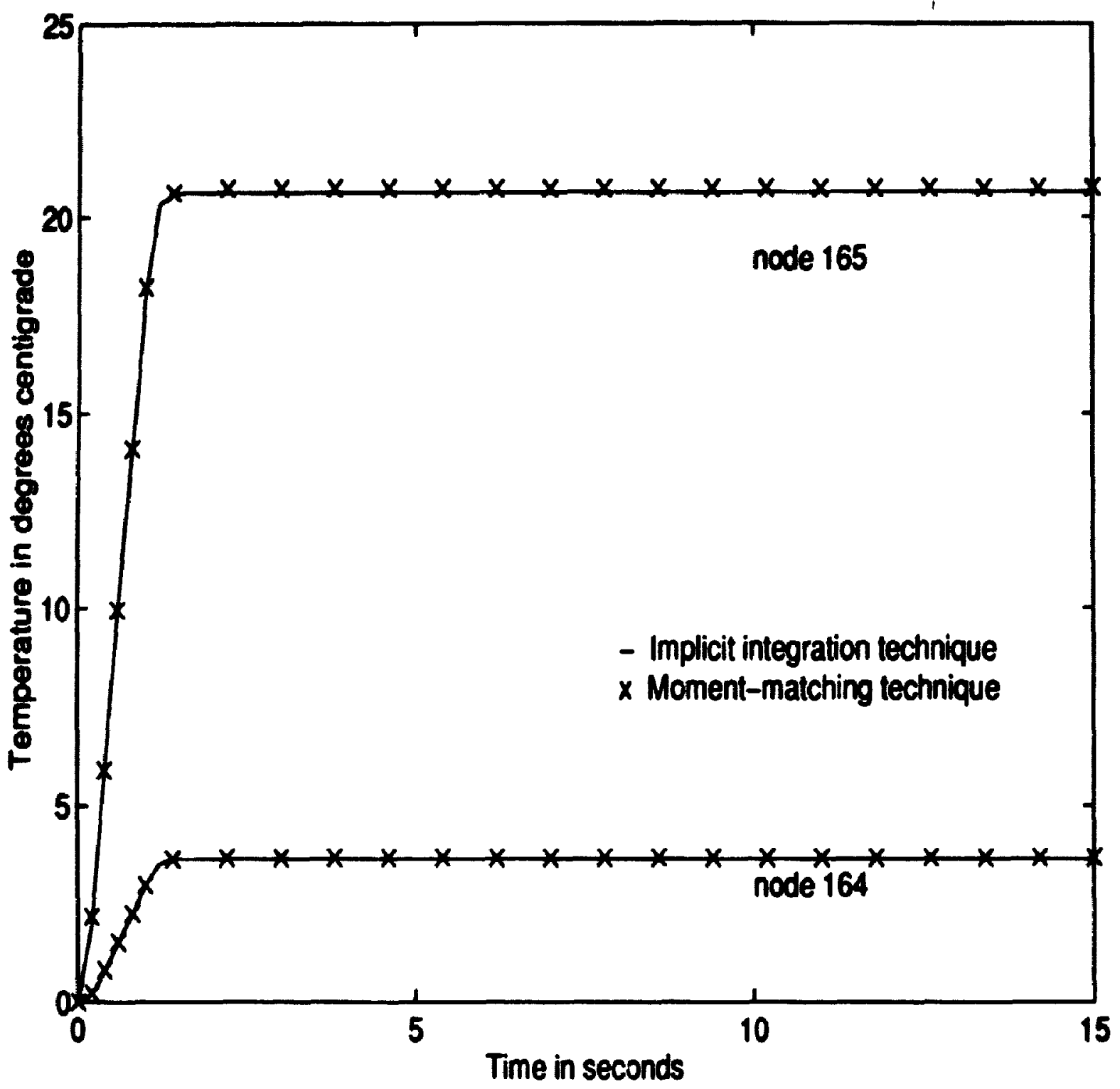

Figure 5.10: Comparison of temperature response using moment-matching and implicit integration techniques for the example in Fig. 5.9 


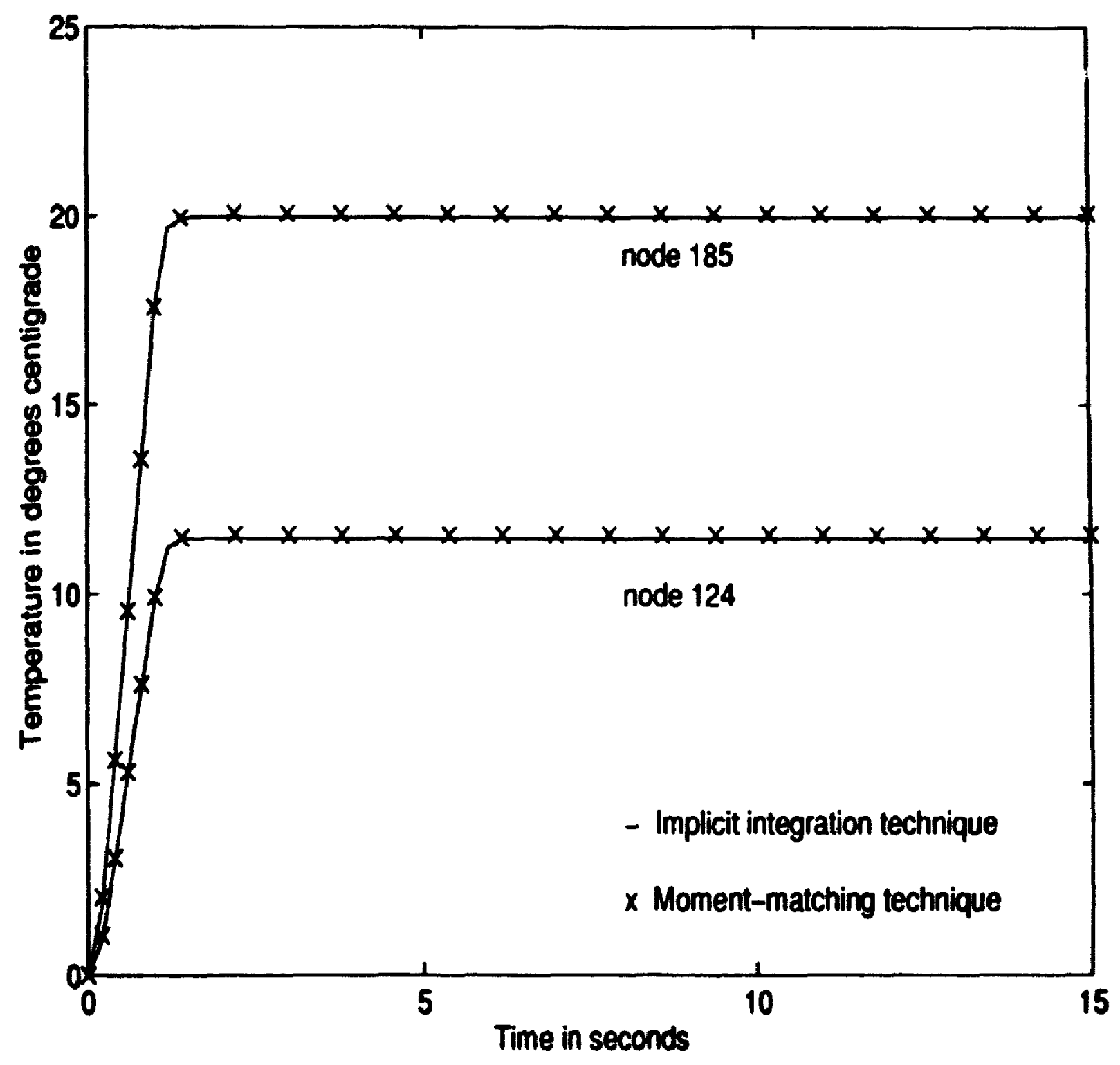

Figure 5.11: Transient temperature response (Example 2) 


\begin{tabular}{lcccccc}
\hline Method & CPU time & \multicolumn{5}{c}{ nodal temperature in $\left({ }^{\circ} \mathrm{C}\right)$} \\
\cline { 3 - 7 } & (seconds) & $T_{124}$ & $T_{144}$ & $T_{164}$ & $T_{165}$ & $T_{185}$ \\
\hline $\begin{array}{l}\text { implicit integration } \\
\text { technique }\end{array}$ & 28.99 & 11.47 & 19.96 & 3.65 & 20.645 & 19.98 \\
$\begin{array}{l}\text { moment matching } \\
\text { technique }\end{array}$ & 0.49 & 11.565 & 20.07 & 3.67 & 20.74 & 20.00 \\
\hline
\end{tabular}

Table 5.2: Temperature solution at $\tau=15$ (seconds) for the example in Fig. 5.9

of circular area required to be equal to the rectangular area of the problem under consideration. The source heat is distributed over two element widths at the package center base. The package leads were modeled by using a thermal conductivity in the appropriate element. Transient temperature response at few selected nodes is shown in Figures 5.10 and 5.11 . Only 5 poles extracted by moment-matching method were sufficient to represent the system. In Table 5.2 a comparison of computational speed using implicit integration tech:sique and the proposed method is given. A speed-up factor of 59 is arhieved using the moment-matching technique.

To study the time domain response due to other possible types of input waveforms, a square pulse is considered. The temperature profiles for a square pulse input are shown in Figures 5.12 and 5.13. Computational statistics for the pulsed input condition are given in Table $\mathbf{5 . 3}$. 


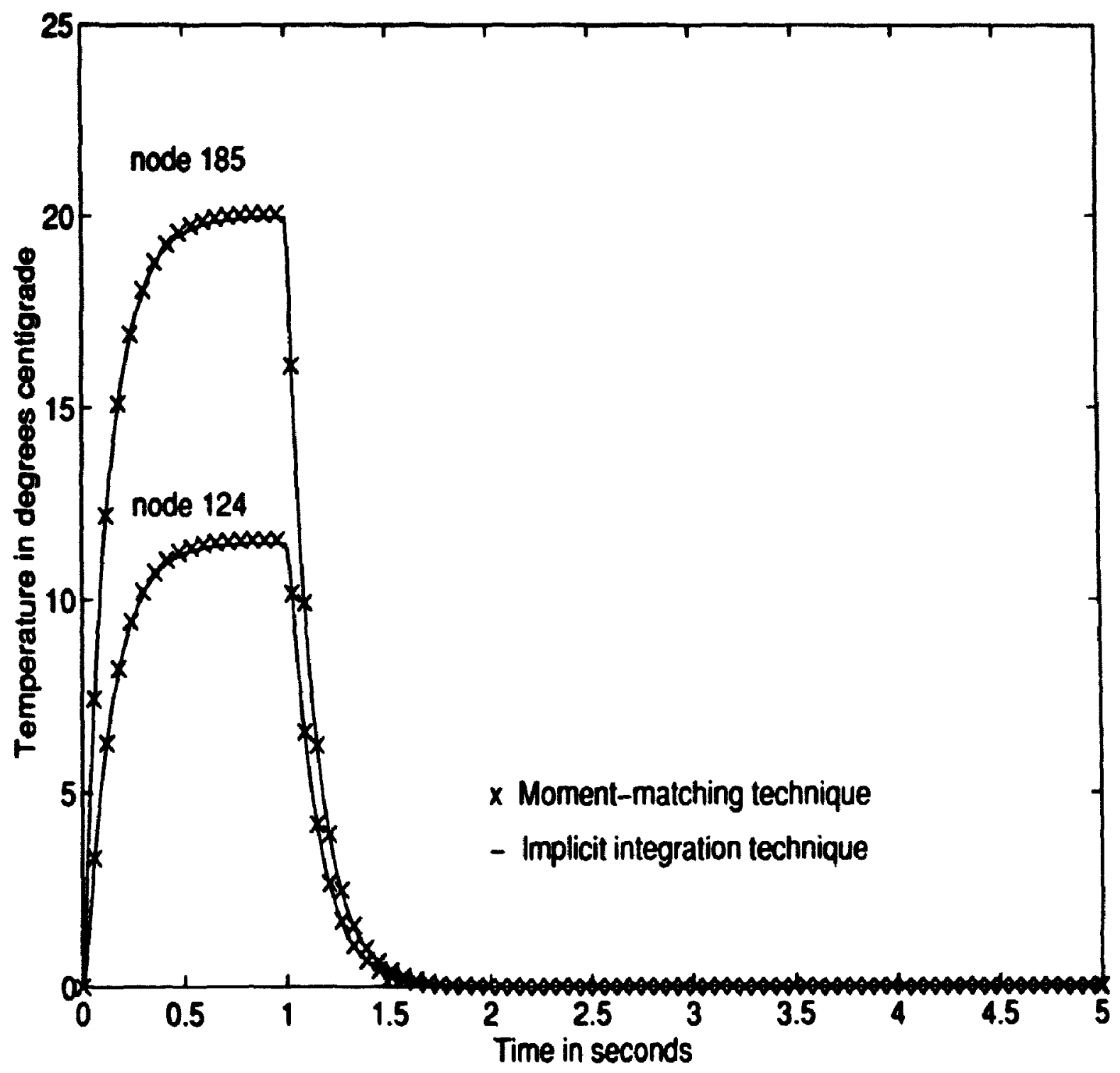

Figure 5.12: Temperature profiles (Example 2) - pulse input 


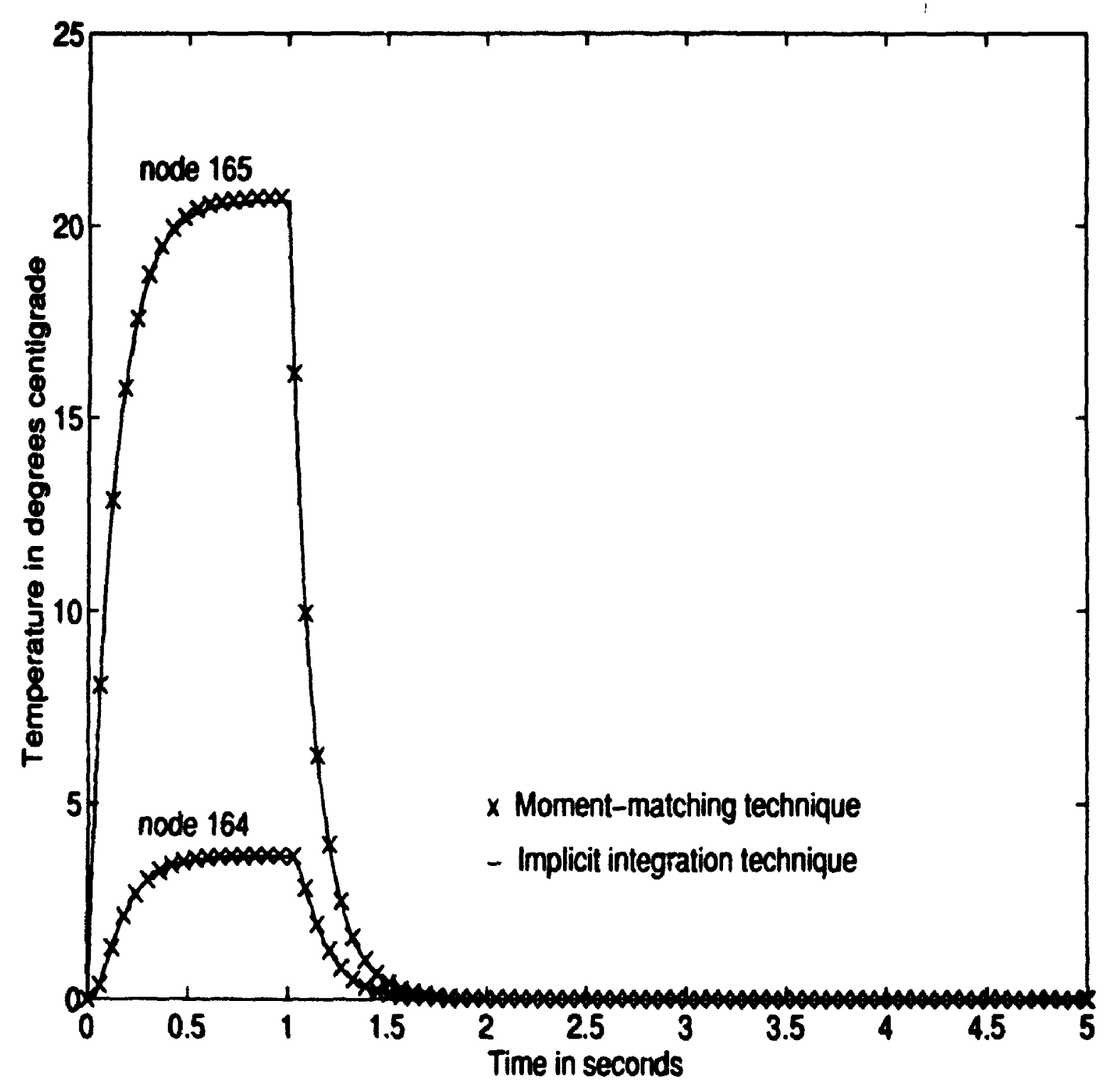

Figure 5.13: Transient temperature response (Example 2) - pulse input 


\begin{tabular}{lcccccc}
\hline Method & $\begin{array}{c}\text { CPU time } \\
\text { (seconds) }\end{array}$ & \multicolumn{5}{c}{ nodal temperature in ("O) } \\
\cline { 3 - 7 } & $T_{124}$ & $T_{144}$ & $T_{164}$ & $T_{165}$ & $T_{185}$ \\
\hline $\begin{array}{l}\text { implicit integration } \\
\text { technique } \\
\text { moment matching }\end{array}$ & 233.0 & 11.47 & 19.96 & 3.65 & 20.645 & 19.98 \\
technique & 0.9 & 11.77 & 20.8 & 3.67 & 20.73 & 20.06 \\
\hline
\end{tabular}

Table 5.3: Temperature solution at $\tau=1$ (seconds) for the example in Fig. 5.9 - pulse input

\subsubsection{Example3: Power Transistors on Panel}

A flat. $0.203 m \times 0.1016 m$ vertically oriented, natural comvection cooled chasis panel dissipates heat from two power transistors [20] as shown in Figure 5.14. 'The' heat dissipation from the base of the transistor is represented by two $0.0127 m x$ 0.012 im surface heat sources. Onr dissipating a heat of 7.511 and the ofler dissi pating 1011. The thermal conductivity of the pancl is $k=1.58 \times 10^{-02} 11 \% 111-"{ }^{\prime}$. The average natural convective heat transfer coeflicient is $h=8.3711 \% m^{2}-. "$ '. 'The finite element model for the chasis panel is shown in Figure 5.15. 'The morlel roulsists of 352 three dimensional shell elements and 391 nodes. 'Transient tenuperature profiles for few nodes are shown in Figure 5.16 and 5.17. (i poles were extrarted by moment-matching technique to represent the system. A romparison of the com putational time using implicit integration at.d moment matching terhniques in given in Table 5.4, which shows that the proposed technique is 18.1 times faster than the iterative technique. 

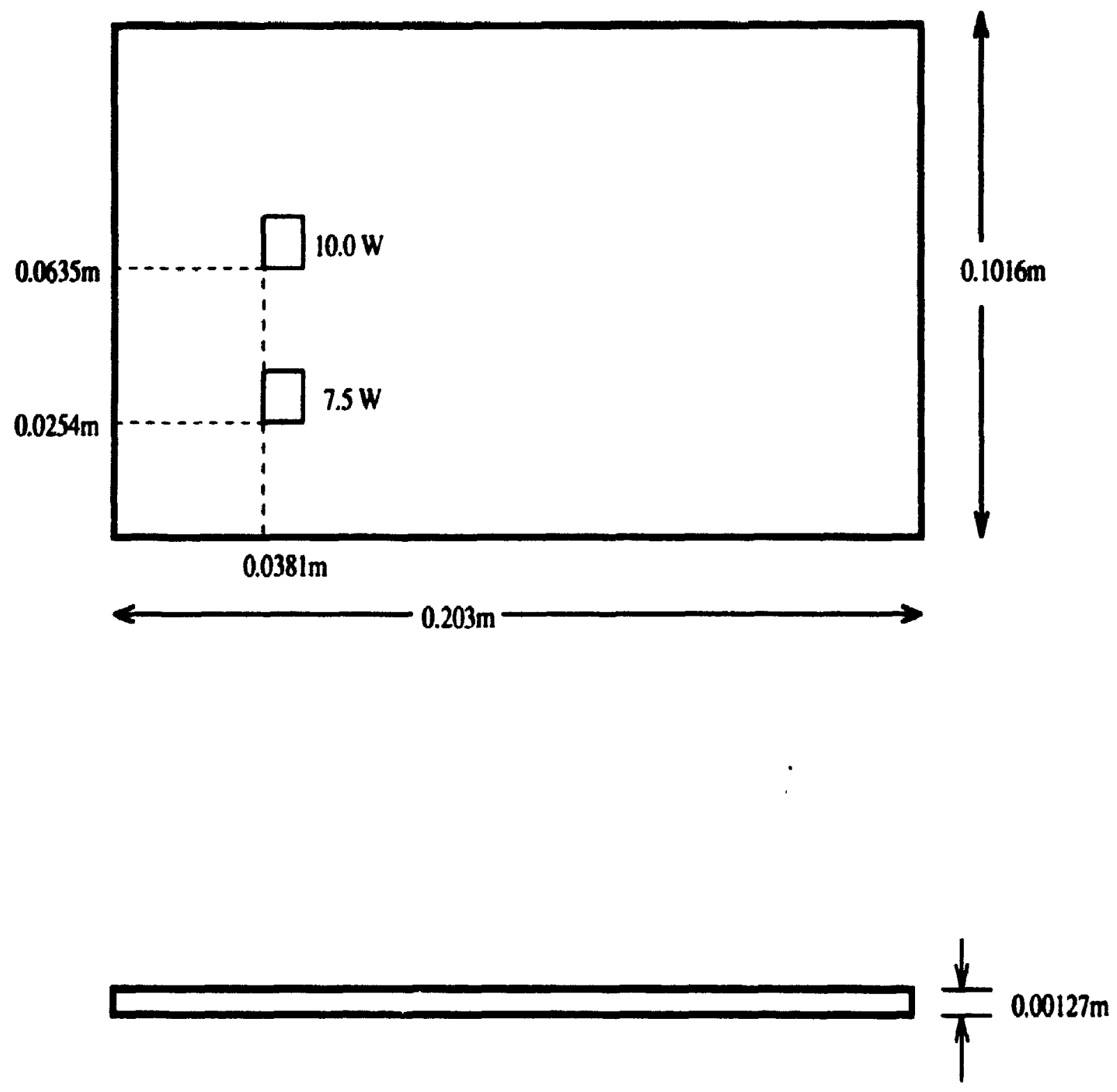

Figure 5.14: Power transistors on a panel 


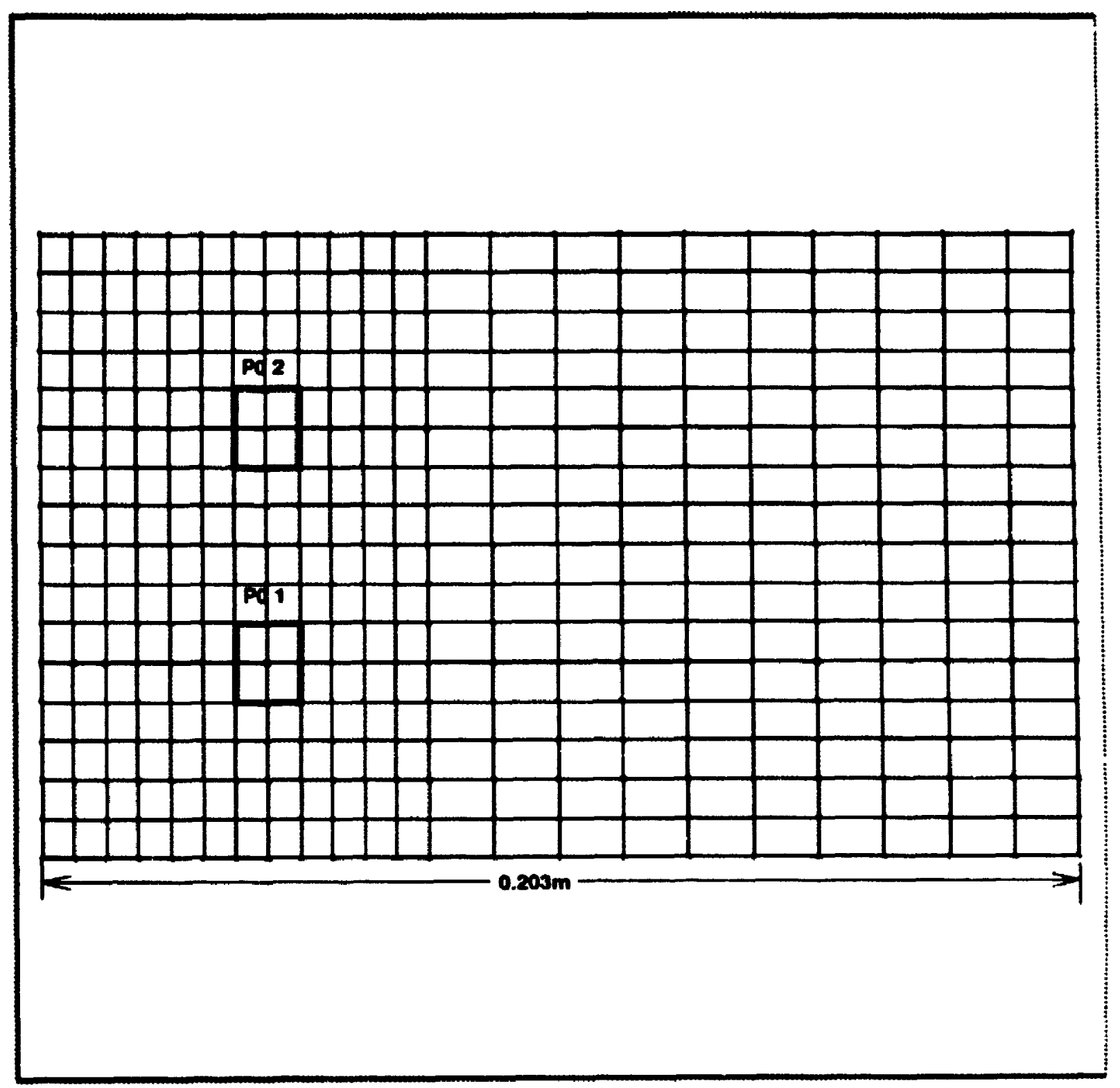

Figure 5.15: Power transistors on a panel - Finite element model 


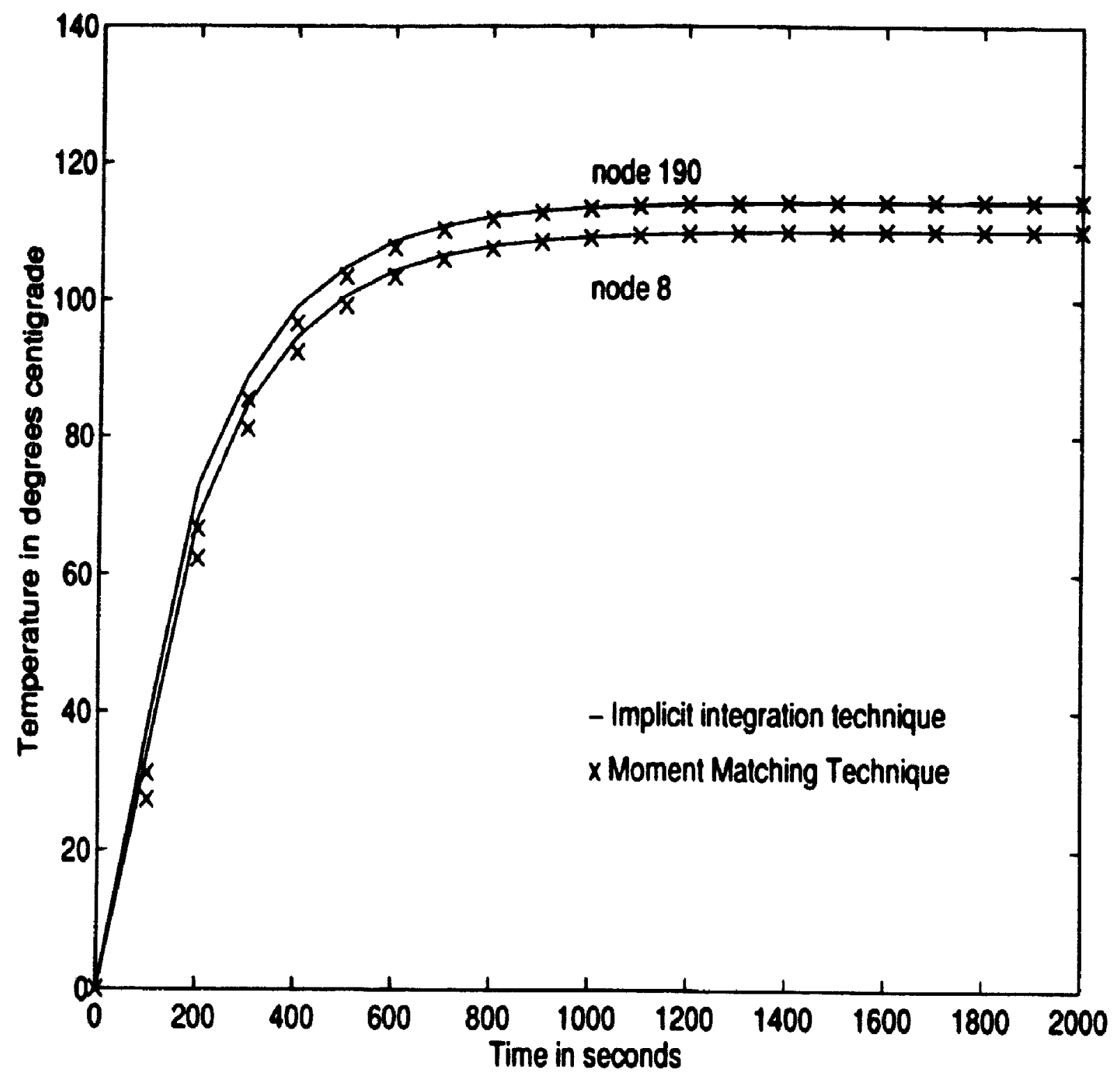

Figure 5.16: Comparison of temperature response using moment-matching and implicit integration techniques for the example in Fig. 5.15 


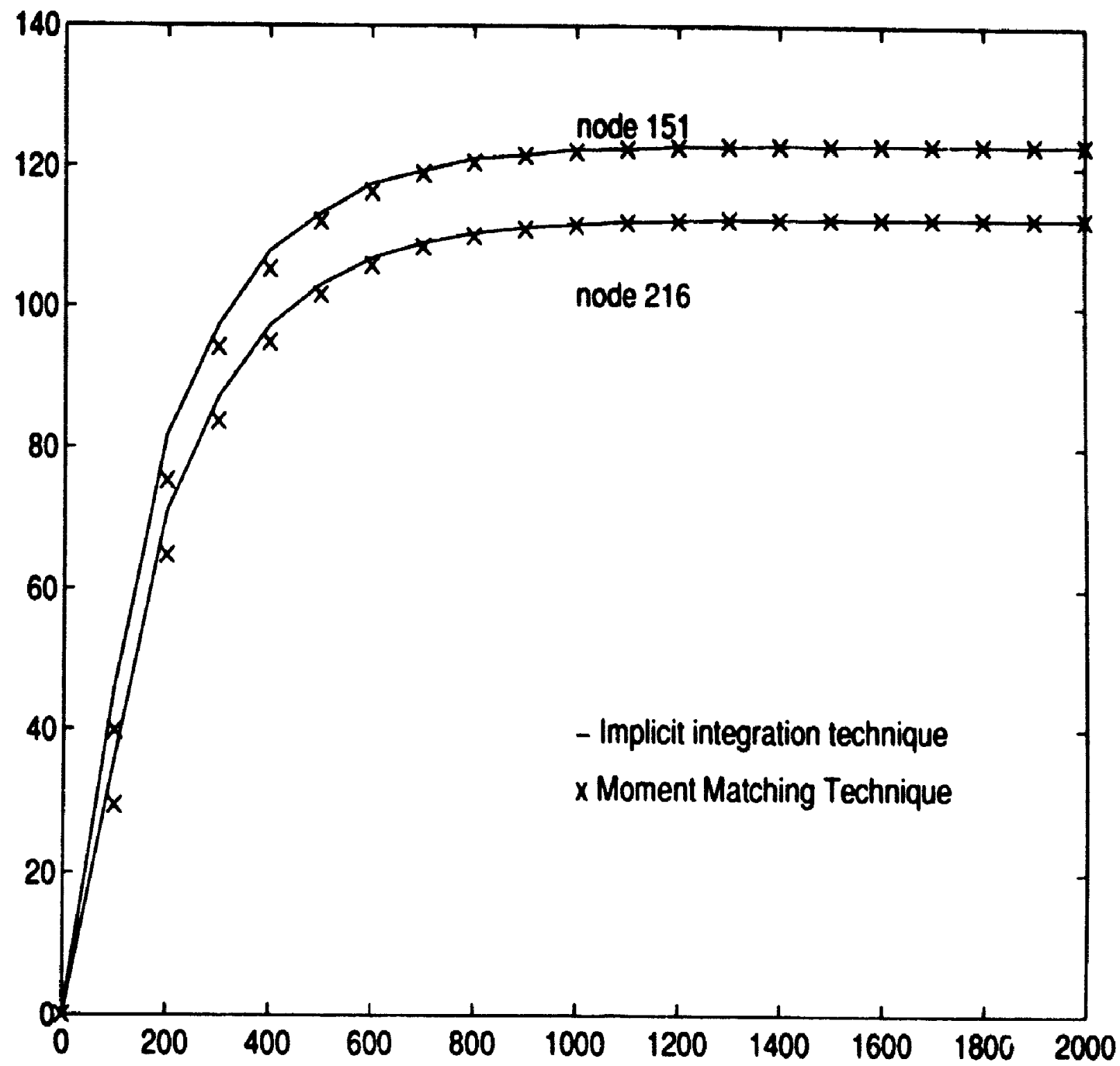

Figure 5.17: Transient temperature response using moment-matching and implicit integration techniques for the example in Fig. 5.15 


\begin{tabular}{|c|c|c|c|c|c|}
\hline \multirow[t]{2}{*}{ Method } & \multirow{2}{*}{$\begin{array}{l}\text { CPU time } \\
\text { (seconds) }\end{array}$} & \multicolumn{4}{|c|}{ nodal temperatur: in $\left({ }^{\circ} \mathrm{C}\right)$} \\
\hline & & $T_{8}$ & $T_{151}$ & $T_{190}$ & $T_{216}$ \\
\hline $\begin{array}{l}\text { implicit integration } \\
\text { technique }\end{array}$ & 363.92 & 110.11 & 123.21 & 114.41 & 112.66 \\
\hline $\begin{array}{l}\text { moment matching } \\
\text { technique }\end{array}$ & 1.98 & 110.12 & 123.14 & 114.40 & 112.67 \\
\hline
\end{tabular}

Table 5.4: Temperature solution for the panel with power transistors

\begin{tabular}{lcc}
\hline Example & Problem Size & Number of Poles \\
\hline 1. & $3625 \times 3625$ & 4 \\
2. & $227 \times 227$ & 5 \\
3. & $391 \times 391$ & 6 \\
\hline
\end{tabular}

Table 5.5: Number of poles extracted by moment-matching technique.

\subsection{Discussion of the Results}

The above set of examples demonst rated several advantages that moment-matching terhnique possesses over the implicit int egration techniques. I'sing moment-matching technique it was sufficient to extract a small set of dominant poles to represent the system behavior, as against calculating all the natural modes. The number of poles extracted for the examples are given in Table. 5.5

The most significant inprovement over the existing techniques is the speed-up attainable. In all the ahove examples the speed-up ratio is $1-2$ orders of magnitude compared to the implicit integration techniques. The accuracy of the results obtained using the proposed technique is very good. The proposed method can be used for parametric studies, which would aid in reducing the number of experiments to be conducted before the production stage. 


\subsection{Summary}

A procedure for transforming the entries in the finite element thermal matrices to electrical network elements was described in order to solve for the system moments using a electrical circuit simulator implementing moment matching method. Considering an example heat conduction problem electrical netlist formulation was explained.

Transient thermal response for a few examples are presented using a new approach which utilizes powerful features of finite element modeling, coupled with an efficient moment-matching technique as a solver. This work has been published as [-15]. 'The. approach is also applicable to more general geometries. ('omparison of results with conventional solution techniques shows substantial improvement in the computational efficiency without any sacrifice in accuracy. 


\section{Chapter 6}

\section{Nonlinear Transient Thermal Analysis Using Circuit Simulation Techniques}

\subsection{Introduction}

Examples of lincar transient thermal analysis of electronic boards/packages using FEM and moment matching technique were discussed in earlier chapter. In this chapter, a methodology for nonlinear transient thermal analysis using Thermal Network Method ( INM) for problem formulation, will be presented. The thermal network is analogous to electrical resistance-capacitance (RC) network. The resistances are for conduction, convection and radiation. For simplicity these elements are modeled as linear elements however, in practice they exhibit nonlinear behavior. The nonlinearity is due to the temperature dependency of convective heat transfer coefficient and thermal conductivity of the material.

A large thermal RC mesh is often required for accurate thermal modeling of electronic systems. Space and time discretisation are used to replace the differential equations governing the heat transfer process by a set of noulinear equations. The 
number of equations being very large. Traditional thermal simulators [1] employ it. erative relaxation techniques such as Gauss-Seidel method, to solve these equations. The two main disadvantages of this method are due to the large number of iterations required for convergence and the need for good initial guess.

Using the thermal network method for the problem formulation. a new approach for nonlinear t:ansient thermal analysis of electronic systems is presented in this chap ter. Nonlinearity due to temperature dependent convective heat transfer coeflicient is considered. Implicit integration techniques and the Newton-Raphson methed $[46]$ are used to solve the resulting differential equations. The integration technique reduces the differential equations to a set of nonlinear difference equations which are solved using the Newton-Raphson method. Derivative information in the form of a Jacobian matrix are utilized to speed up convergence. The technique can be easily implemented using conventional circuit simulators.

\subsection{Problem Formulation}

Several boundary conditions could be encountered in heat transfer problems. Convective boundary condition is applied for the solution of a heat conduction problem when a solid is bounded by a convecting fluid. In this case, the bunndary condition to be satisfied by the temperature field in the solid is

$$
-k\left(\frac{\Delta T}{\Delta n}\right)_{s}=h\left(T_{s}-T_{j}\right)
$$

where $\Delta T$ is the temperature change at the boundary, "is the out ward drawn normal at the surface. $h$ is convective heat transfer coefficient. $T_{s}$ is the temperature of the solid, and $T_{f}$ is the temperature of the fluid. 
Estimation of the convective heat transfer coefficient $h$ is considered to be an important aspect in air cooled electronic systems. A number of empirical ccrrelations are available in the literature $[17,17,48]$ for the evaluation of $h$. The evaluation of $h$ depends on the physical properties(density, viscosity, etc.) of the fluid past a given geometry and the geometrical configuration itself. The nonlinear behavior of $h$ is due to the significant dependence of the physical properties of the fluid flow on temperaturc. For different printed circuit board (PCB) and electronic package configurations, convective heat transfer coefficient calculations are given in [3].

Using the thermal net work method the nodal network can be represented in three dimension, as shown in Figure 6.1, the thermal resistance $R_{t}$, and capacitance $C_{3}$ can be evaluated as follows

$$
\begin{gathered}
R_{k(1, j)}=\frac{l_{t j}}{k \times A_{k_{1 j}}}, \text { for conduction } \\
R_{i}(i j)(T)=\frac{1}{h_{i j}(T) A_{s i j}}, \text { for convection } \\
C_{t}=l_{i} \rho c_{p}
\end{gathered}
$$

where $R_{1}$, is the thermal resistance, $l_{1}$ is the conduction distance between node $i$ and $j, C_{t}$ is the thermal capacitance, $A_{k ı}$ is the cross-sectional area for heat conduction normal to $l_{i}, V_{1}$ is the volume around node $i, \hat{q}_{1}$ is heat generated or added to volume $V_{i}, h_{\mathfrak{t}}(T)$ is the temperature dependent heat transfer coefficient between node $i$ and $j, A_{i i}$, is the surface area of the body exposed to convecting fluid.

Approximating the derivatives in the three dimensional heat conduction equation (2.8) by finite differences. the general equation of the nodal network [17] with time and space discretization for the explicit and implicit cases are, 


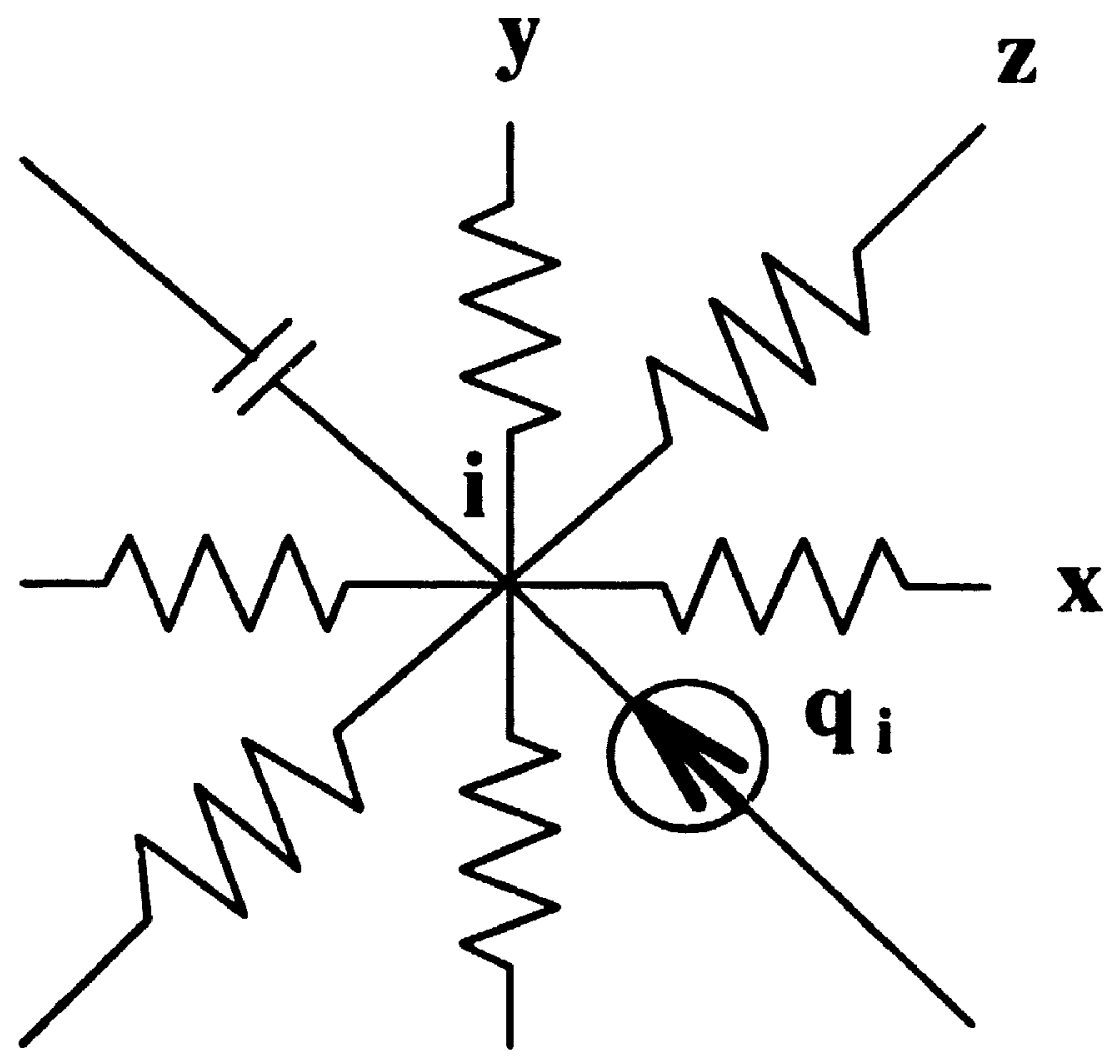

Figure 6.1: 3-dimensional thermal network at nedre, 


$$
\begin{aligned}
& \sum_{,} \frac{\left(T_{j}-T_{s}\right)}{R_{i j}}+\hat{q}_{i}=C_{i} \frac{\left(T_{i}^{\prime}-T_{i}\right)}{\Delta \tau} \ldots \text { explicit } \\
& \sum_{j} \frac{\left(T_{j}^{\prime}-T_{i}^{\prime}\right)}{R_{i j}}+\hat{q}_{i}=C_{i} \frac{\left(T_{i}^{\prime}-T_{i}\right)}{\Delta \tau} \ldots \text { implicit }
\end{aligned}
$$

where $j$ denotes all neighboring nodes connected to node $i . T_{i}, T$, are the temperatures at nodes $i, j$ at time $\tau . T_{i}^{\prime}, T_{j}^{\prime}$ are the temperature at time $\tau+\Delta \tau$.

For the implicit case using $(6.4)$ the temperature at time $\tau+\Delta \tau$ can be determined by,

$$
T_{1}^{\prime}=T_{1}+\Delta \tau\left(\sum_{j} \frac{T_{j}^{\prime}-T_{i}^{\prime}}{R_{1} C_{1}}+\frac{\dot{q}_{1}}{C_{2}}\right)
$$

In the circuit simulation based approach [46], the original thermal equation (2.8) with convective boundary conditions (6.1), for a thermal RC network with $\boldsymbol{N}$ nodes, is reduced to a set of nonlinear ordinary differential equations.

$$
\mathbf{C} \dot{\mathbf{T}}+\mathbf{G T}+\phi(\mathbf{T})=\mathbf{b}(\tau)
$$

where

$$
\begin{aligned}
& C \in \Re^{N \times N} \\
& \mathbf{C}=\operatorname{diag} .\left\{\begin{array}{llll}
C_{1} & C_{2} & \cdots & C_{N}
\end{array}\right\} \\
& G \in \Re^{N \times N} \\
& \mathbf{G}=\sum_{i=1}^{N} \sum_{j=1}^{N} \frac{1}{R_{\mathrm{i} j}}\left(\mathbf{U}_{\mathrm{i}}-\mathbf{U}_{\jmath}\right)\left(\mathbf{U}_{\mathrm{t}}-\mathbf{U}_{\mathrm{j}}\right)^{t} \\
& \phi(\mathbf{T}) \in \Re^{N} \\
& \phi(T)=\sum_{(1, j)} \phi_{1},(T)\left(\mathbf{U}_{\mathrm{t}}-\mathbf{U}_{\jmath}\right)^{t} \\
& (i, j) \in I_{p p}
\end{aligned}
$$


where $I_{p p}$ is the set of node pairs for all nonlinear resistors $R_{c(1 \jmath)}(T)$. Considering nonlinearity due to temperature dependent convective heat transfer coefficient.

$$
\begin{aligned}
& \phi(T)=\frac{T_{i}-T}{R_{c(i j)}(T)} \\
& \mathbf{U} \in \Re^{N} \\
& \mathbf{U}_{i}=\left[\begin{array}{lllllll}
0 & \cdots & 0 & 1 & 0 & \cdots & 0
\end{array}\right]^{t} \\
& \mathbf{b} \in \Re^{N} \\
& \mathbf{b}=\left[\begin{array}{llll}
\hat{\boldsymbol{q}}_{1} & \hat{\boldsymbol{q}}_{2} & \cdots & \hat{\boldsymbol{q}}_{N}
\end{array}\right]^{\mathrm{t}} \\
& \mathbf{T} \in \Re^{N} \\
& \mathbf{T}=\left[\begin{array}{llll}
T_{1} & T_{2} & \cdots & T_{N}
\end{array}\right]^{\mathrm{t}} \\
& \dot{\mathbf{T}}=\frac{d \mathbf{T}}{d \tau}
\end{aligned}
$$

$t$ denotes transpose

\subsection{Solution of Network Equations Using Implicit Integration and Newton-Raphson Method}

Using implicit integration technique sach as the Trapezoidal Rule $[49,46]$, solutiom at time $\tau_{n+1}$, can be written as

$$
\mathbf{T}_{n+1}=\mathbf{T}_{n}+\frac{\delta}{2}\left(\dot{\mathbf{T}}_{n}+\dot{\mathbf{T}}_{n+1}\right)
$$

where $\delta$ is the time step size and

$$
\begin{aligned}
\mathbf{T}_{n} & =\mathbf{T}\left(\tau_{n}\right) \\
\mathbf{T}_{n+1} & =\mathbf{T}\left(\tau_{n+1}\right)
\end{aligned}
$$


Multiplying (6.7) by $\mathrm{C}$ gives,

$$
\mathrm{CT}_{n+1}=\mathbf{C} \mathbf{T}_{n}+\frac{\delta}{2}\left(\mathbf{C \mathbf { T } _ { n }}+\mathbf{C \mathbf { T } _ { n + 1 }}\right)
$$

eliminating the derivatives in (6.8) using (6.6) results in a set of algebraic equations,

$$
\begin{gathered}
\left(\mathbf{C}+\frac{\delta \mathbf{G}}{2}\right) \mathbf{T}_{n+1}-\left(\mathbf{C}-\frac{\delta \mathbf{G}}{2}\right) \mathbf{T}_{n}+ \\
\frac{\delta}{2}\left\{\phi\left(\mathbf{T}_{n}\right)+\phi\left(\mathbf{T}_{n+1}\right)\right\}-\frac{\delta}{2}\left\{\mathbf{b}_{n}+\mathbf{b}_{n+1}\right\}=0
\end{gathered}
$$

where

$$
\begin{gathered}
\mathbf{b}_{n}=\mathbf{b}\left(\tau_{n}\right) \\
\mathbf{b}_{n+1}=\mathbf{b}\left(\tau_{n+1}\right)
\end{gathered}
$$

Denoting the vector of variables by $T$ and the vector of functions by $f,(6.9)$ can be written in a compact form as,

$$
\mathbf{f}(\mathbf{T})=\mathbf{0}
$$

Assuming that the system has a solution and denoting the solution by $\mathbf{T}^{*}$ and expanding each function in a Taylor series about $\mathbf{T}$ :

$$
\begin{aligned}
f_{1}\left(\mathbf{T}^{*}\right) & =f_{1}(T)+\frac{\partial f_{1}}{\partial T_{1}}\left(T_{1}^{*}-T_{1}\right)+\cdots \frac{\partial f_{1}}{\partial T_{1}}\left(T_{n}^{*}-T_{n}\right) \\
f_{2}\left(\mathbf{T}^{*}\right) & =f_{2}(T)+\frac{\partial f_{2}}{\partial T_{1}}\left(T_{1}^{*}-T_{1}\right)+\cdots \frac{\partial f_{2}}{\partial T_{1}}\left(T_{n}^{*}-T_{n}\right) \\
& \vdots \\
f_{n}\left(\mathbf{T}^{*}\right) & =f_{n}(T)+\frac{\partial f_{n}}{\partial T_{1}}\left(T_{1}^{*}-T_{1}\right)+\cdots \frac{\partial f_{n}}{\partial T_{1}}\left(T_{n}^{*}-T_{n}\right)
\end{aligned}
$$

Assuming that $\mathbf{T}$ is close to $\mathrm{T}^{*}$ higher order terms may be neglected and the system may be described in linearized form: 


$$
\mathbf{f}\left(\mathbf{T}^{*}\right) \approx \mathbf{f}(\mathbf{T})+\mathbf{J}\left(\mathbf{T}^{*}-\mathbf{T}\right)
$$

where

$$
\left.\mathbf{J}\right|_{\mathbf{T}}=\left[\begin{array}{cccc}
\frac{\partial f_{1}}{\partial T_{1}} & \frac{\partial f_{1}}{\partial T_{2}} & \cdots & \frac{\partial f_{1}}{\partial T_{n}} \\
\frac{\partial f_{2}}{\partial T_{1}} & \frac{\partial f_{2}}{\partial T_{2}} & \cdots & \frac{\partial f_{2}}{\partial T_{n}} \\
\vdots & \vdots & \vdots & \vdots \\
\frac{\partial f_{n}}{\partial T_{1}} & \frac{\partial f_{n}}{\partial T_{2}} & \cdots & \frac{\partial f_{n}}{\partial T_{n}}
\end{array}\right]_{\mid \mathbf{T}}
$$

is the Jacobian matrix of the function $f$. If equation $(6.12)$ is set to zero and solved, the result will not be the vector $\mathbf{T}^{*}$ (because the higher order terms have been neglected) but some new value $T$. Using superscript $I$ to denote the iteration number. the solution at iteration $I$ and time step $\tau_{n+1}$ is

$$
\mathbf{J}^{\prime} \Delta \mathbf{T}_{n+1}^{\prime}=-\mathbf{f}\left(\mathbf{T}_{n+1}^{\prime}\right)
$$

where

$$
\Delta \mathbf{T}^{\prime}=\mathbf{T}^{\prime+1}-\mathbf{T}^{\prime}
$$

and

$$
\mathbf{T}_{n+1}^{\prime+1}=\mathbf{T}_{n+1}^{\prime}+\Delta \mathbf{T}_{n+1}^{\prime}
$$

Formulae (6.14) and (6.16) represent the Newton- Raphson algorithm for a system of equations.

From (6.9) Jacobian matrix can evaluated using.

$$
\mathbf{J}=\left(\mathbf{C}+\frac{\delta \mathbf{G}}{2}\right)+\left.\frac{\partial \phi}{\partial \mathbf{T}}\right|_{\mathbf{T}_{n+1}^{\prime}}
$$

\subsection{Results}

To illust rate the solution methodology discussed in the previous sections, all example of a forced air conled hybrid package is given as shown in Figure 6.2. The 


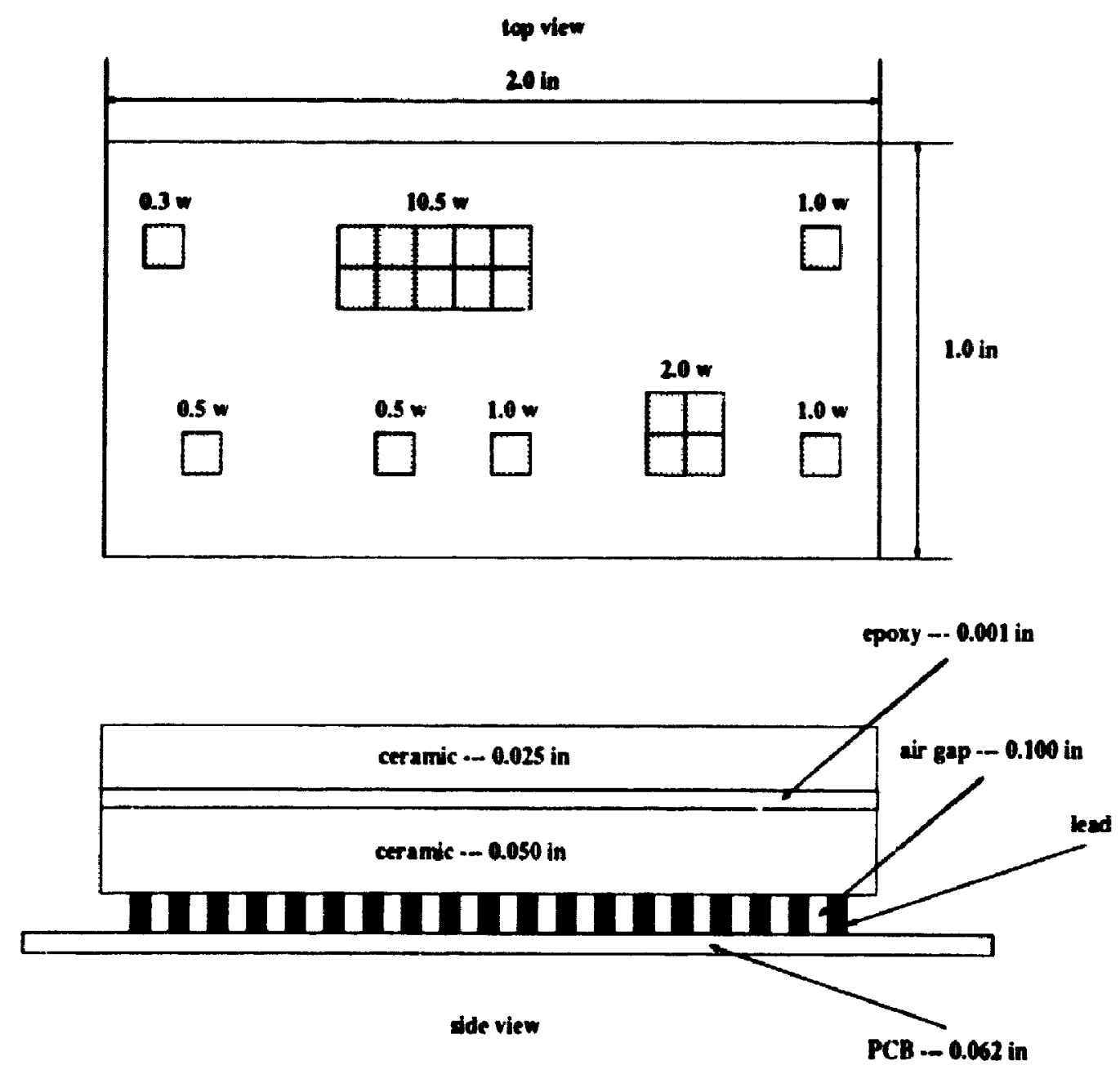

Figure 6.2: Hybrid circuit package geometry [1] 
hybrid package is a ceramic-epoxy-ceramic structure [1] and has a top surface source plane, with 19 leads side brazed to each edge of the lower ceramic layer. Figure 6.3 shows a thermal network model for the hybrid package which is divided into thres layers. Each layer consists of 200 nodes resulting in 600 temperature nodes on the hybrid package. The ambient air is assigned to node 601 . The thermal network components are calculated following [1]. The top layer convective resist ances are replaced with nonlinear convective resistances. The empirical formula representing temperature dependency of heat transfer coefficient was taken from [3]. Temperature profiles at various nodes were obtained using a circuit simulator employing NewtomRaphson method and the implicit integration technique for the solution. Also, the solution was obtained using a thermal sinulat or [1] employing (iauss-Seidel method assuming an initial condition of $20^{\circ} \mathrm{C}$ for all the nodes at time $\tau=0$.

The transient temperat ure response at a few selected nodes of the thermal net work are shown in Figure 6.4. A comparison of simulation results using the approach presented in this chapter and relaxation approach [1], is given in Figure 6.5, which shows that the two responses are in good agreement. Computational statisties for the transient analysis of the hybrid package using the solution methodology pressented in Section 7.3 and relaxation technique, are shown in Table 6.1. The approach using, implicit integration techniques and Newton-Raphson method has taken less (PP time compared to the relaxation technique. For the example shown, the present approach is found to be 3 times faster than the iterative relinxation approach. 


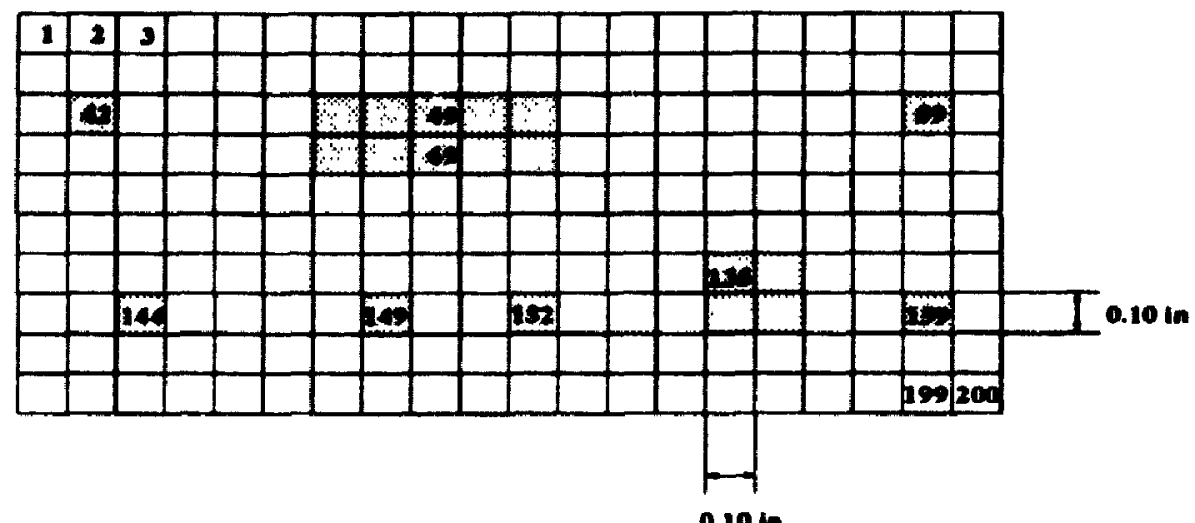

(a)

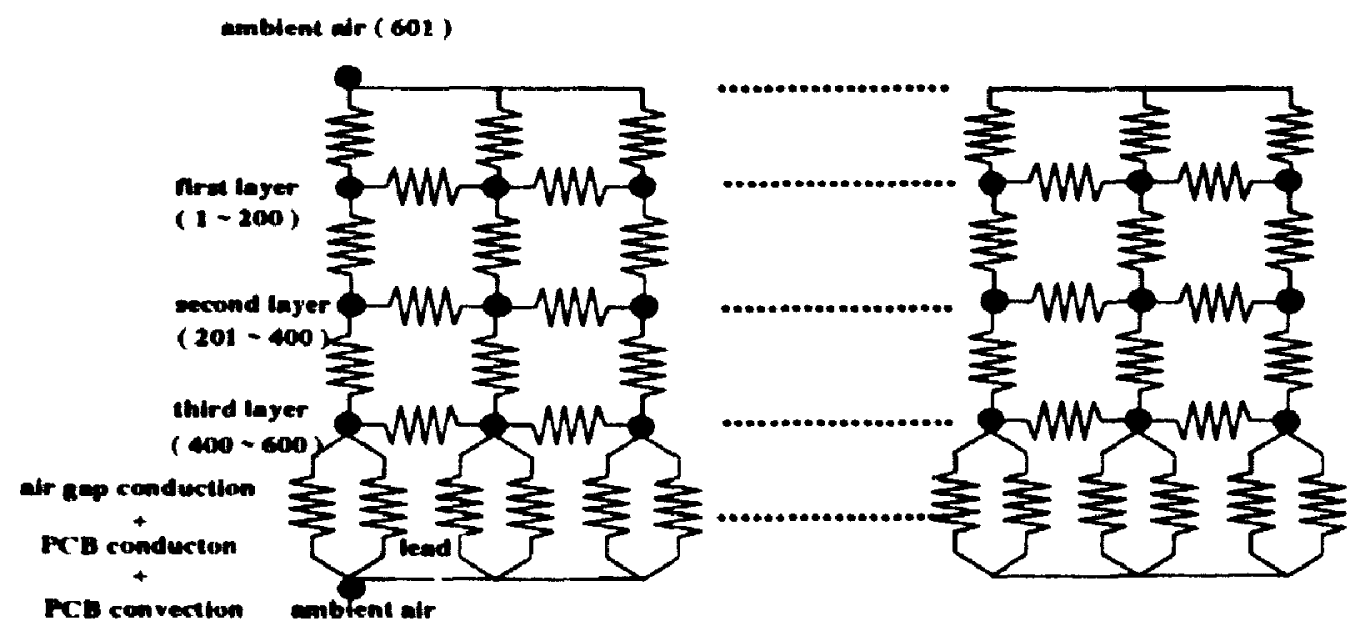

(b)

Figure 6.3: Thermal network model showing thermal resistors for the hybrid circuit package (a) Top view (b) Side view 


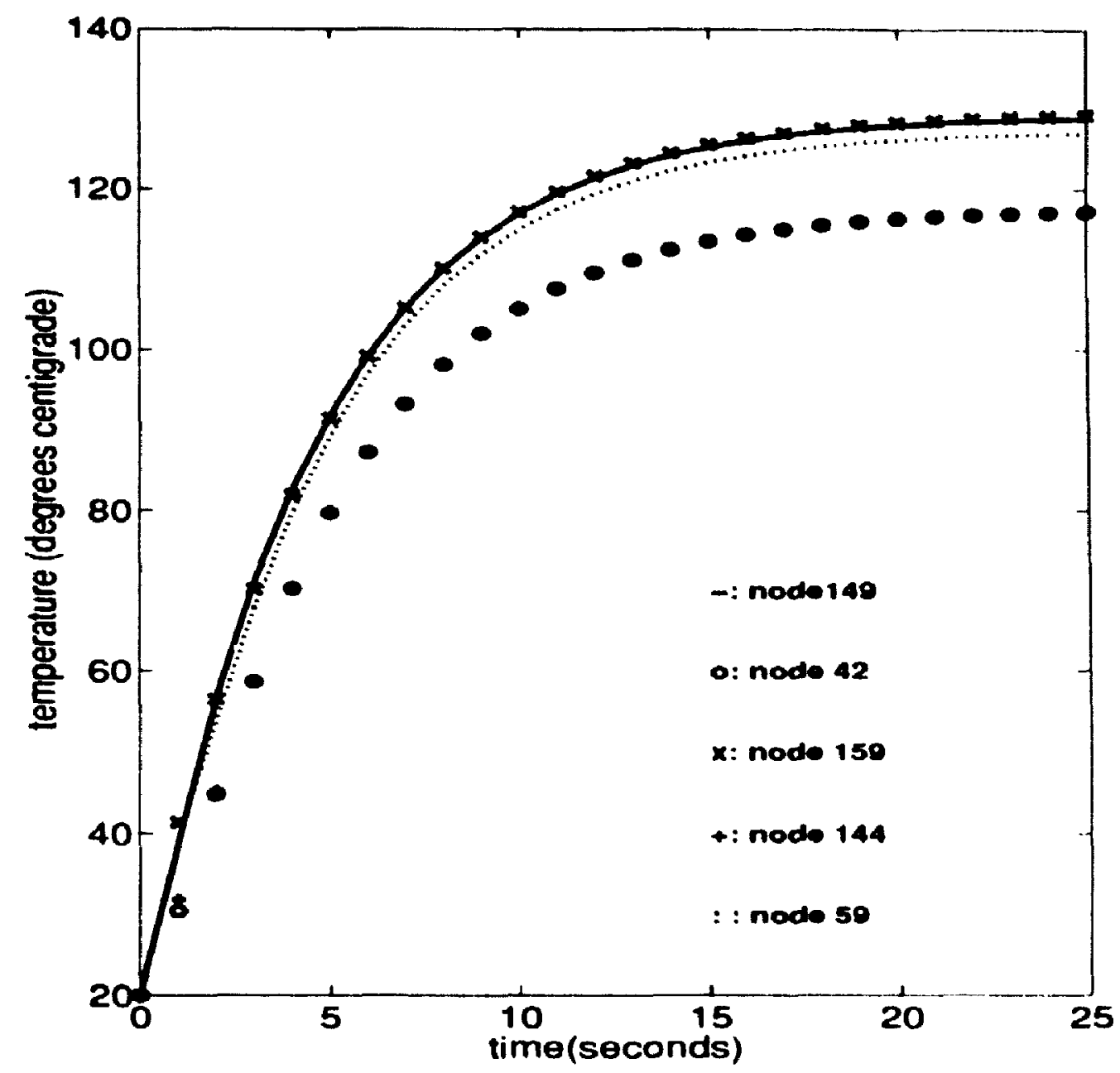

Figure 6.4: Temperature profiles using Newton-Raphson method and implicit inte gration technique 


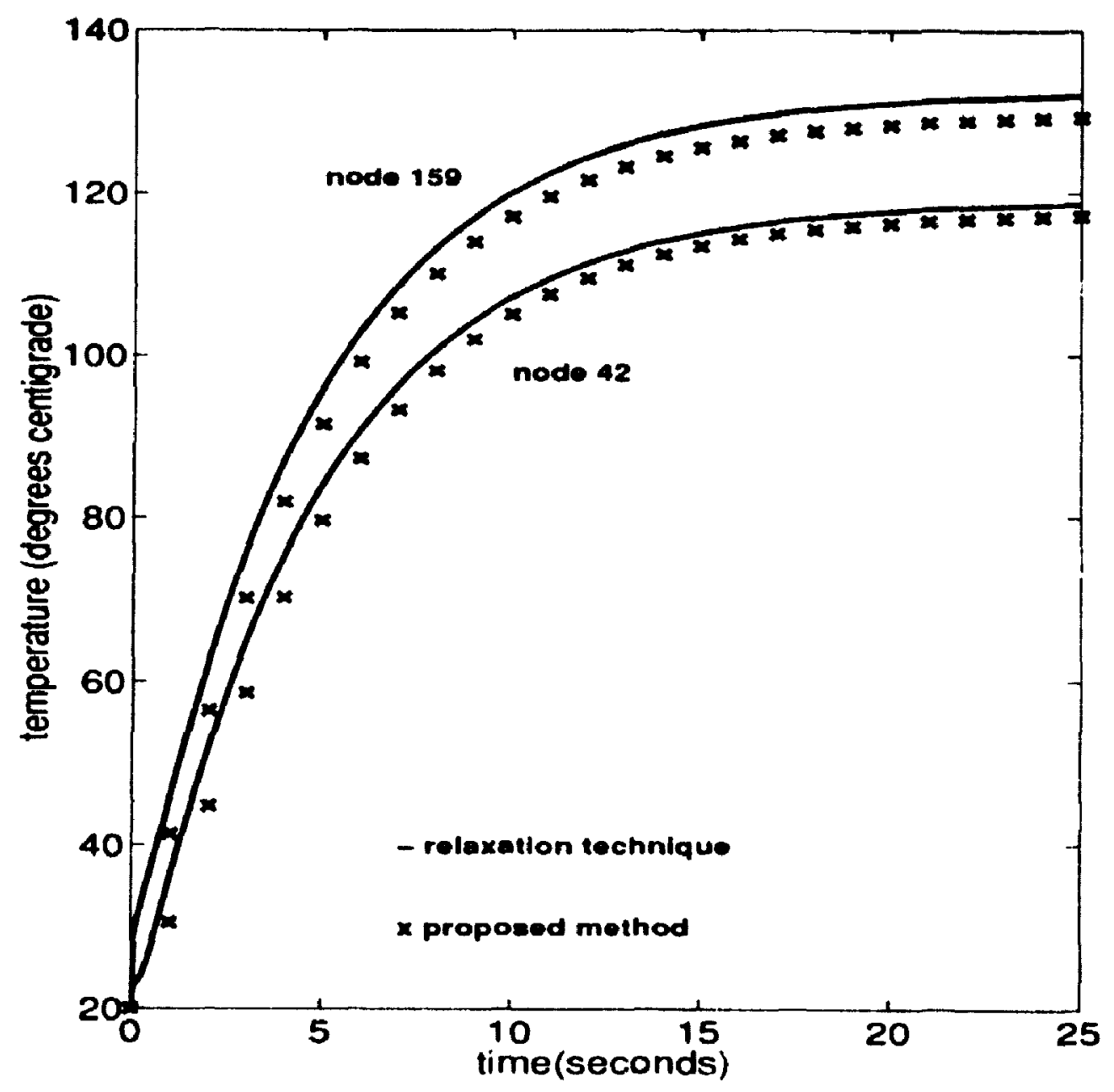

Figure 6.5: ('omparison between Newton-Raphson technique and relaxation technique" 


\begin{tabular}{|c|c|c|c|c|c|c|}
\hline \multirow[t]{2}{*}{ method } & \multirow{2}{*}{$\begin{array}{l}\text { CPl time } \\
\text { (seconds) }\end{array}$} & \multicolumn{5}{|c|}{$\begin{array}{l}\text { nodal temperat ure in (" }(\mathrm{)}) \\
\text { at } \tau=25 \text { (seconds) }\end{array}$} \\
\hline & & $I_{42}$ & $I_{59}$ & $T_{144}$ & $T_{14 !}$ & Tisn \\
\hline $\begin{array}{l}\text { relaxation } \\
\text { approach [1] }\end{array}$ & 379.0 & 118.68 & 130.27 & 119.20 & $12 ! 1.26$ & 131.97 \\
\hline $\begin{array}{l}\text { present } \\
\text { approach }\end{array}$ & 128.01 & 117.23 & 127.0 .1 & 117.15 & 125.91 & 129! \\
\hline
\end{tabular}

Table 6.1: Nodal temperature solution for the hỵrid package.

\subsection{Summary}

In this chapter a new apposach for thermal modeling of elect ronic systeme is pre

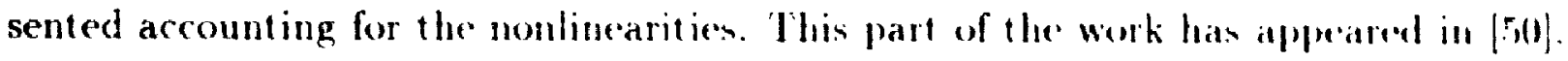
The problem was formulated uning thermal Re metwoth which was solved unimg, me plicit integration technigues and the Xewten-kaphon method. An example of at

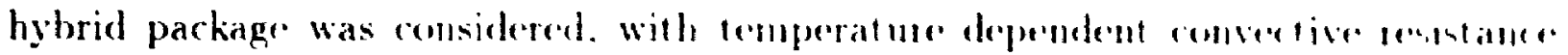

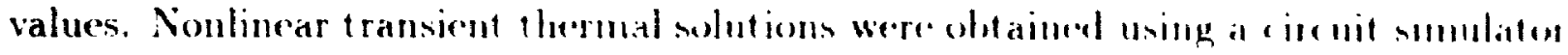
and a standard thermal simmlator based on itcrative relaxation techmiefue. A com parison between temperature profiles obtained using both simulators shows that the results are in good agrement. Ising the apploseh presented in this chapher, it is observed that improved convergence rate and higher computational eflie ienty an he. achieved. Also, it is observed that a range of thermal denign problemue can le eash solved using available circuit simulators. 


\section{Chapter 7}

\section{Conclusions}

\subsection{Summary}

In this thesis two new approarhes for the linear and nonlinear transient thermal analysis of electronic systems are presented. Both approaches using electrical circuit simulation techniques for the solution.

For linear transient thermal analysis, examples of electronic boards/packages were formulated using FEM. The moment-matching method was used for solving the resulting finite element equations. Fsing the moment matching method resulted in a speed-up of 1-2 orders in comparison to the comventional iterative techniques. For all the examples, a comparison between the proposed and the implicit integration tech. nique was made. It was shown that the moment matching method, doesn't require discretization in the time domain for the calculation of the time domain response. which is otherwise considered to be a major jssue in conventional integration techniques where it is often necessary to take small time steps to avoid numerically induced oscillations.

For nonlinear transient analysis. circuit simulation techniques were used. Transient temperature results for an example of a hybrid package are presented using 
the thermal network method for formulation of the probleme. Nonlinearity due to convective heat transfer coefficient was ronsidered.

\subsection{Scope for Further Work}

Considering the import ance of thermal design for opt imum electrical performante of electronic packages. the technique's presented in thesis can li: used for connurrent electrical and thermal analyses. In wher words finite element thermal analysis pank age and electrical circuit simulatol packitge can be interfared te perform dymamis electro-thermal analynis of a tange of electronic packigges. Mlow. with the under of

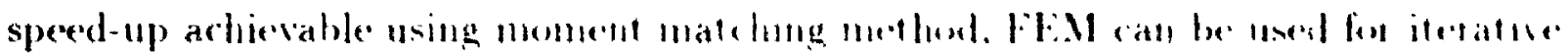
design process without any difficul!!

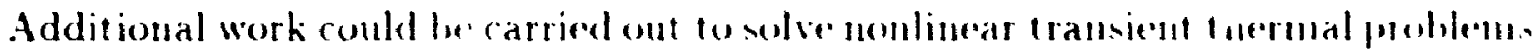
using the combination of FE.M and moment matching techoigue propmed in the thesis. 

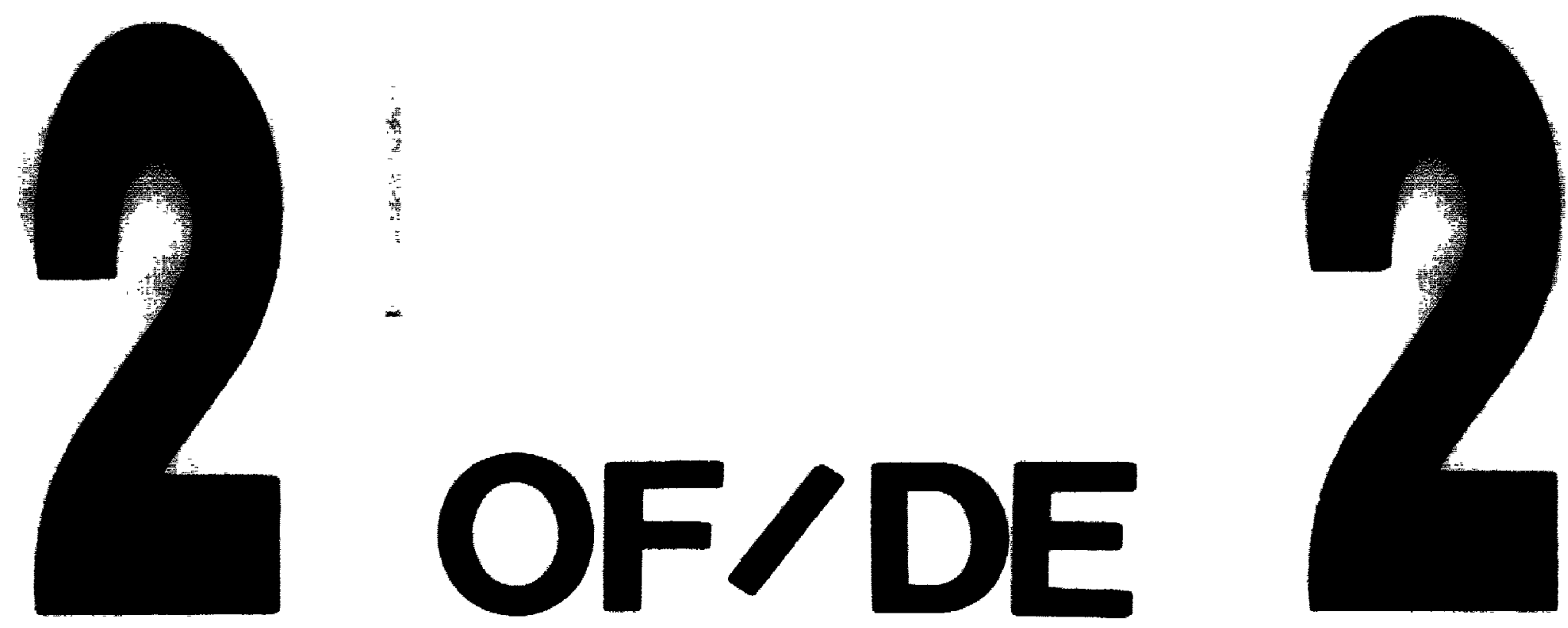

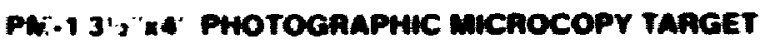
ites rovo Anst/1SO 2 EOUNALENT

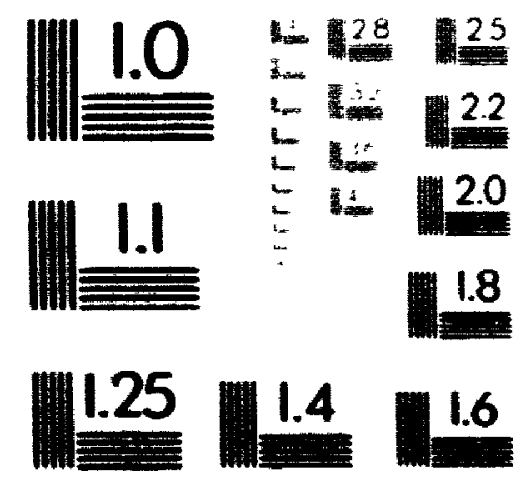

PRECrenon mesolutnow taReETs 


\section{Bibliography}

[1] G. X. Ellison. ETAP - Electronics Thormal Analysis Package: Thermal Analysis with Affordable Programs. Tektronix. Inc. Computer Science ('enter. Heaver ton. Oregon. 1993.

[2] A. Bar-Cohen and A. D. Kraws. Adrances in Thromal Modeling of litectrome Components and Systems. ASML press. Xow York. 19M:3.

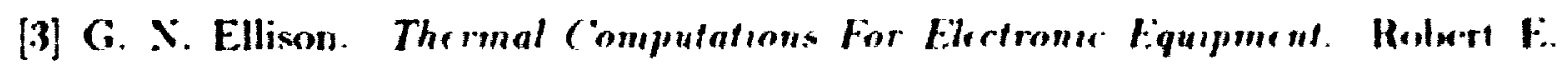
Krieger. Malabar. Florida. $19 \mathrm{~N} 9$.

[4] R. Hsu. The Finte Eloment Metherd an Thermanertaners. Nllen and I'nuin. Boston. 1990.

[5] M. Ibrahim, L. Paradis, and D. Prterson. "Finite element modelling of MMIC" trasmitter module for thermal/strurtural design optimizalion". IH:E Truns. Components. Hybrids. Manuf. Terhnol... vol. 15:723 729. IMY2.

[6] G. E. Myers. "The critical step for finite element solutions to two dimensional heat conduction transients". A.S.ME J. Heat Tran.sfor. vol. 100:1201 127. 1978.

[7] G. E. Myers. Analytical Mrthods in Conduction Meat Transfer. Mr(iraw-Ilill. Sew York. 1978. 
[s] I. T. (he's atsd (: K. ("he't. -IIybrid Laplace transforn/finite element met hod for tuo dimensional transient heat conduction". Journal of The rmophysics. vol. 2:21 36. 19xi.

[9] C. K. Chen and T. M. Chen. Tren hybrid Laplace transform/finite element method for three-dimensional trancient heat conduction problem". Int. J. Num. Mrth. Eng.. vol. 3:1:15 61. 1!1!1!

[11] T. M. Che'n. A modified hybrid laplace transform/finite element transient heat conduction problems". ("omp. Meth. App. Mtrot. Eng. vol. 95:261-272. 1992.

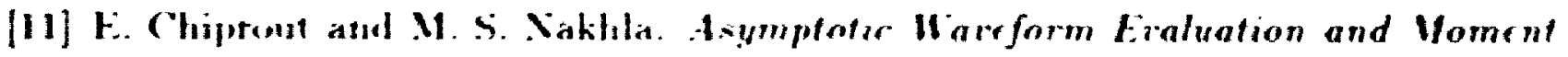
Matchong for Interommet Analy-s.s. Kluwer Academic Publishers. Boston. MA. 1901.

[12] S. Lin and E. S. Kuh. Trausirnt simulation of lossy interconncets based on the recursive comwolution furmulation". IEEE Transactoms on C̈̈ruits and systems I: Fundawental Throry and Application. vol. 3!1no. 11):599 592. Xov. 1942.

[1:3] I.. T. Pillage and R. A. Rohrer. "Asymptotic waveform evaluation for timing analysis". IEEF: Transaction. on (omputer-Aided Drsign. Vol. 9(no. 4):352-366. 1990.

[11] VISA - Numerically Intrgrated Elements for System A nalysis. Engineering Mechanics Research Corporation. Troy. Mirhigan . CSA.. 1992.

[15] FEAl'-Finite HI me nt Analysis of Pronted Circuit Boards. Fnginerering Merhanics Research Corporation . Troy. Michigan . I'SA.. 1992. 
BIBI.IOGRAPIIY

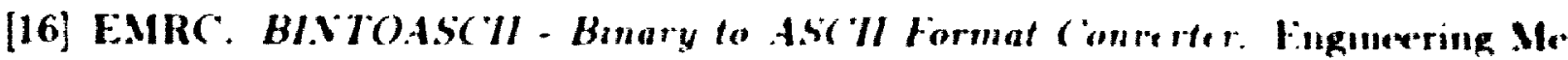
chanics Research Corporation. Tron. Michigan .ISA. Itkats

[1i] A. J. Chapman. Fundamentuis of He at Transfer. Macmillan Publishing company: Rice Iniversity. Texas. I'S.A. 198T.

[18] J. H. Lein ard. A Heat Transfor Tost Book. Prentice Hall line Heglewimed ( 'liffs. N.J. 195 .

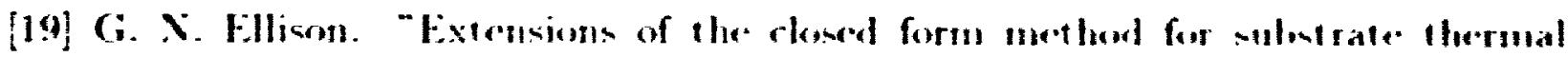

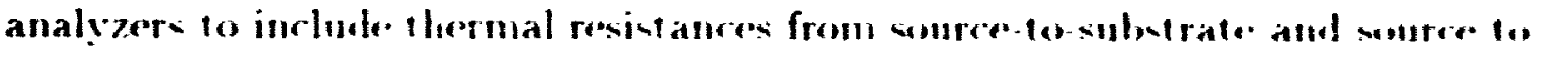

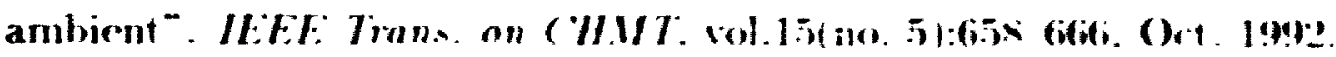

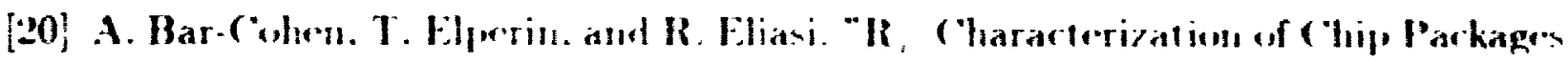
- Iustification. limitations. and Furure". HEE trams. Comp. Hubrod. Monuf

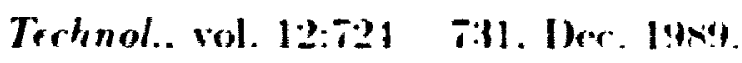

[21] W. K. Krueger and A. Har-(Cohen. Thermal Clatacterizatien of a Ploce

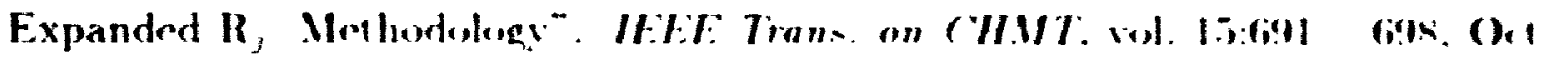
1992.

[22] Cian Ó Mathuna and D. (i. Liu. "Derelopment and application of test hipe and test systems for the thermal characterisation of IC packaging techmokgies". Procedings 8th IEEE Se mi-The rm Symposium. pagm 1i N. March. IMg2.

[23] R. S. Vogelsong and ('. Brezezinski. "Foxtending SPIC ' l: for elect ro-thermal sim

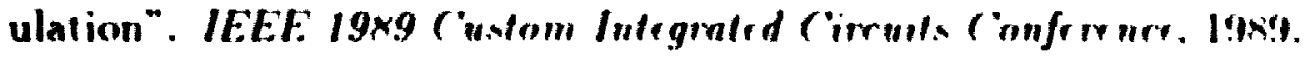


[24] J. F. ("hon and (iary Yip. "SPIC $\mathrm{C}$ models for thermal and vibration analysis of printed circuit boards". Procedeng. of the 27th Interuncity Energy ("oncersion Engentrong ronforruct. I!9?2.

[25] A. R. Hefuer and D. L. Blackburn. Thermal component nodels for electrothermal network simulation". Proredings of SE.HITHEK.M 93. 1993.

[20i] X. K. Verghese. S.S Lare and J. J. Allstut. "A unified approach to simulating elect rical and thermal wubst rate coupling interaction in ICs". 1st conference on (Al). pages 12: 126. Now 1993.

[27] D.(i. Lin. N.Phanilatha. Q.J. Thang. and M.S.Xakh]a. "Asymptotic Thermal Analysis of Printed ("ircuit Boards". IttE. Trans. Components Packages and

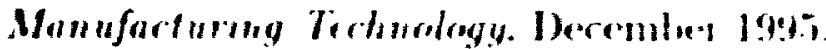

[2N] S. Subramanian and II. D. Rolph. Thermal analysi of direct chip mount electronir systems". Coputers and Structure. vol. 32(no. 3/4):85.3 859, 1989.

[29] W, F. Pence and J. P. Krusius. "Parkage thermal resist ance: geometrical effects in convent ional and hybrid packages". HEEE Trans. on (W.ITT. vol. 13(no.2):2.45 251. 1990.

[30] B. K. Simon. Y. Yuan. and J. R. Imaretịa. "Parametric study of a VLSI plastic using locally refined finite element models". Fifth IEEE SEMIIHER.M symposium, pages $52-58.1989$.

[31] R. Sullahan. M. Fredholm. T. Monaghan. and A. Agarwal. "Thermal mod. eling and allalysis of pin grid arrays and multichip modules". Sereth IEEE SEMITHEKM symposium, pages 110-116. 1991. 
[32] A. M. Anderson and R. I. Moffert. "A heat transfer currelation for arbitrars geometries in elct ronic equipment ${ }^{-}$. ASUE: unter annual moteng. Mallas. TA. 1990.

[33] K. Azar and E. T. Russell. "Effert of component layout and geometry on the flow

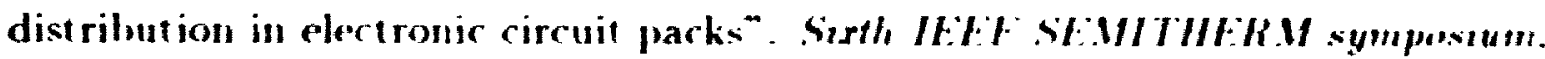
pagem 1-10. 1990.

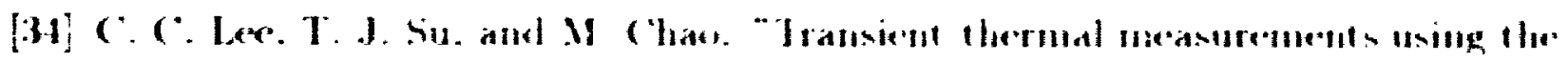
index of refraction an a temperature sensitive parameter". H:T lian cimp

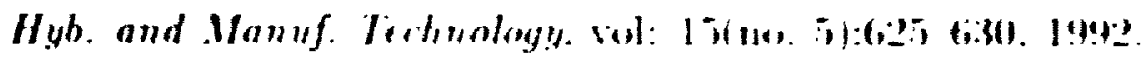

[35] J. X. Funk. M. Pinar Menguc. K. Azar. Tagav. and r..l Cremern. "A semi

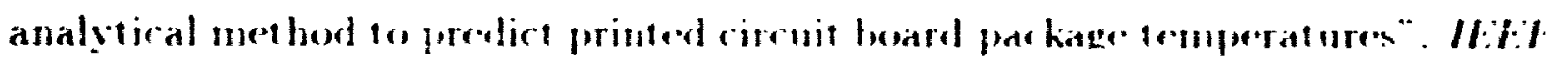

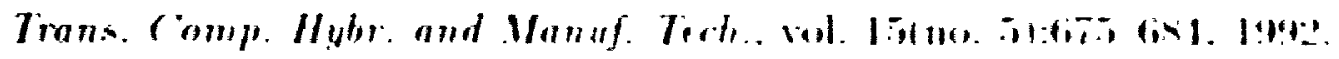

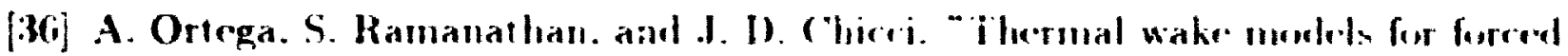

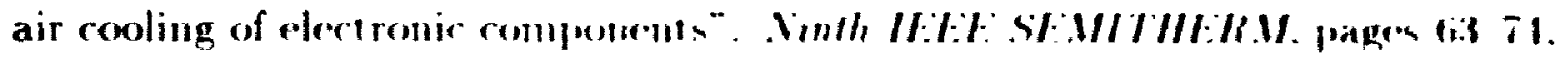
1993.

[37] Y. J. Min. A. L. Palisoc, and (C. C'. Lee. "Transient t!ermal study of semicom ductor devices". IEEE: Tran. ('mmp. Hyb. and ifanuf. Technolngy. vol: I:3(me 4):980-988. 1990.

[38] .. A. Brebbia and .1. Domingurz. Boundary Filements, An Introdurtory Course. Computational Merhanics Pulblications. Beston. Crepublisherd with Mr (iraw IIill Book Company. New York. I9s?. 
[39] O.C.Zienkiewicz. The Fimte Element Method. Tata MrGiaw Hill. New Delhi. 1990 .

[40] R. W. Lewis. K. Morgan. and O. (' Zienkiewicz. Numoral Methods in Heat Transfer. Wiley. Toronto. 1981.

[41] R. D. Cook. D. S. Malkus, and M. F. Plesha Concepts and Applications of

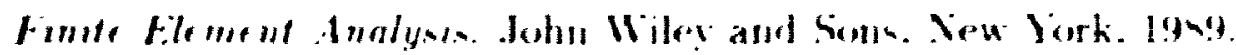

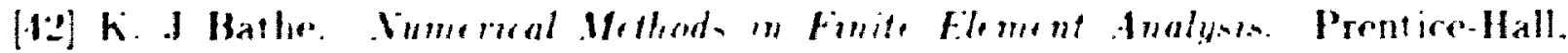
Finglenomed ( liff. N.I. 1976.

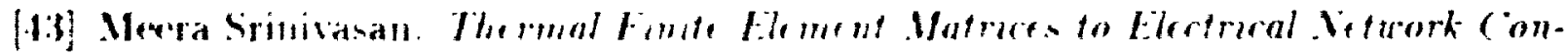

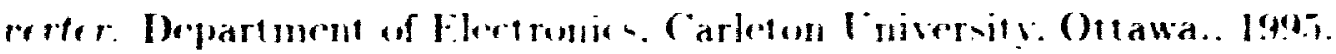

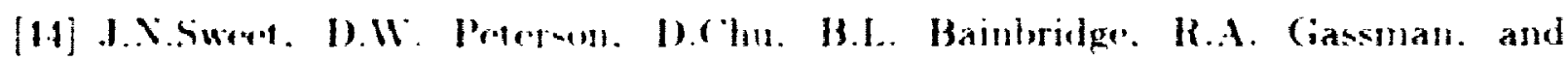
r.A.Relere "Analyis and Meanurement of Thermal Resistance in a 3 -

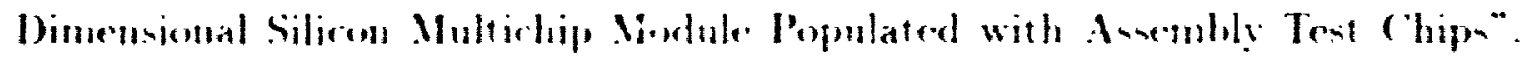

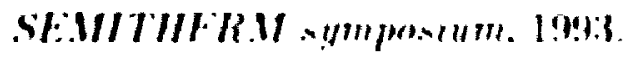

[45] V.Phanilatha, D.(B. Liu. M.S.Nakhla, and Q.J.Zhang. "Finite Element Thermal Analysin of Electronic Boards and Packages I sing Moment Matching Tech. niques". ITHEKMTOG. 19!6.

[10] J. Varh and $\mathrm{K}$. Singhal. Computer Mtthods for Circuit Analysis and Design. Van Nostrand Reinhold. New York. 1983.

[17] W..M hays and M.F. Crawford. Convetime heat and mass transfer. MrCiraw-Hill. 1950. 


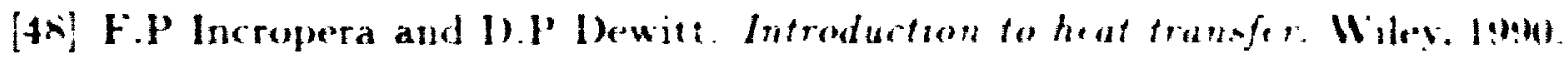

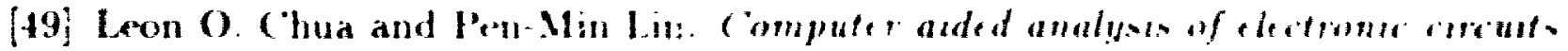

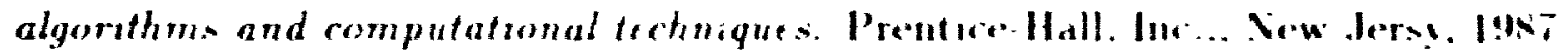

[50] V.Phanilatha. D. (i. Liu. Q. J. Zhang, and Mi. S. Nakhla. Minhloner transient

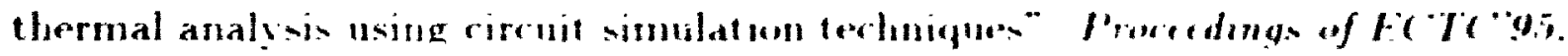
Mas. 1:19: 


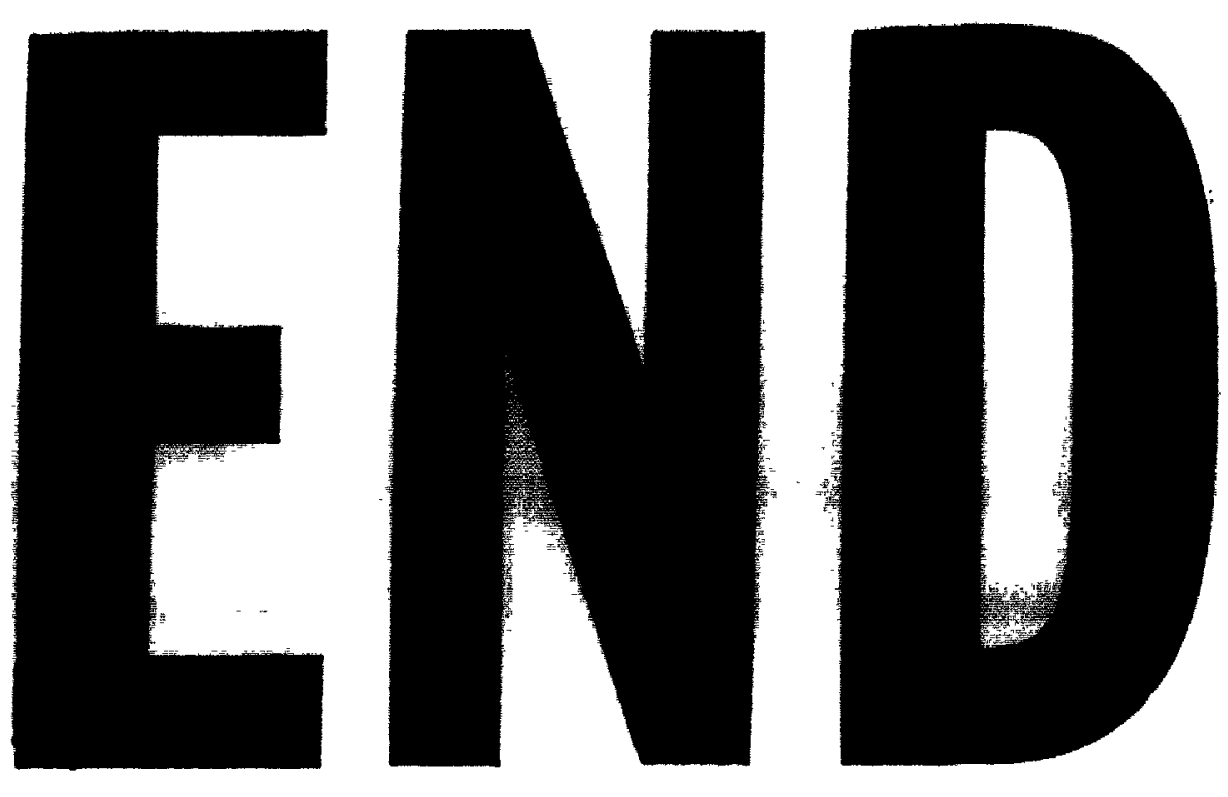

$19+06.07$
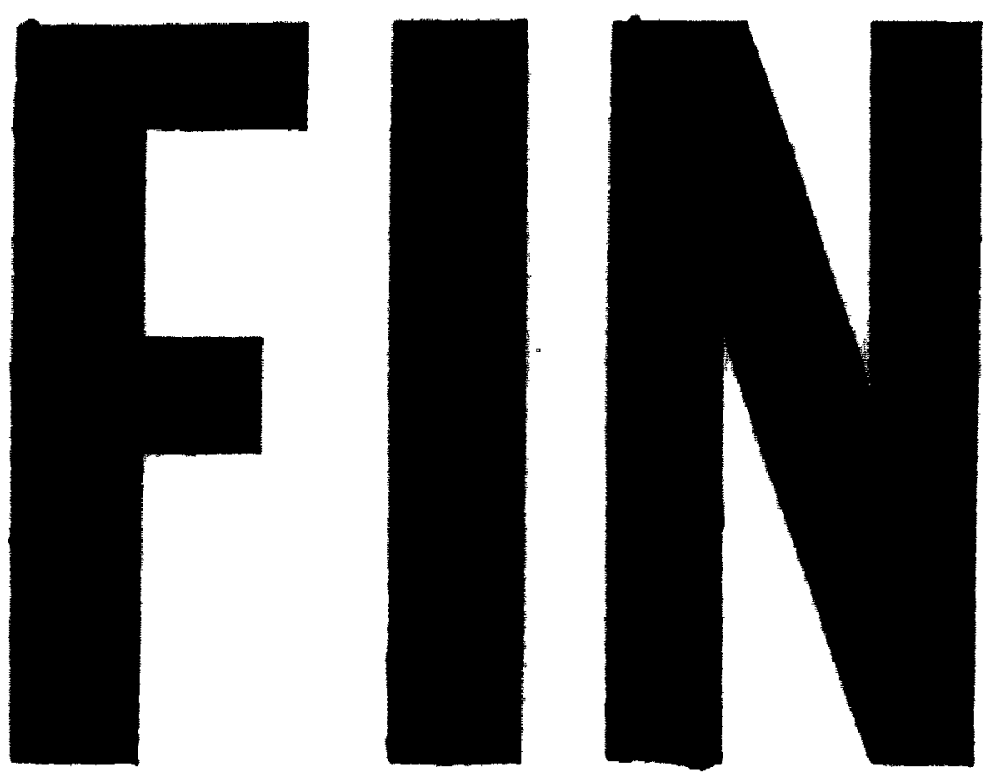